\title{
ASPECTOS DE REPARAMETRIZAÇÃO NAS INFERÊNCIAS SOBRE A CONFIABILIDADE DE SISTEMAS.
}

\author{
José Carlos Fogo
}

Orientador: Prof. Dr. Jorge Alberto Achcar

Dissertação apresentada ao Instituto de Ciências Matemáticas de São Carlos USP, como parte dos requisitos para obtenção do Título de Mestre em Ciências Área: "Ciências de Computação e Matemática Computacional".

USP - São Carlos

Agosto de 1994. 
Dedico este Trabalho aos meus Pais Vilézio e Antonia e a minha esposa Elza. 
Dificilmente existe algo tão impressionante para a imaginação como a admirável forma da ordem cósmica expressa, pela "Lei da Frequência do erro (Distribuição Normal)". Tivessem os gregos conhecido, certamente a teriam personificado e deificado.

Sir Francis Galton 


\section{Agradecimentos}

A conclusão dessa dissertação não seria possível sem o auxílio de algumas pessoas e instituições que de alguma forma contribuiram para este projeto, às quais, expresso meus sinceros agradecimentos.

Ao Prof. Dr. Jorge Alberto Achcar pela orientação sempre dedicada e pelo incentivo em todo o decorrer do projeto de pesquisa.

À FAPESP e CNPq pelo apoio financeiro.

À Profa. Dra. Maria Creusa Bretas Salles e ao Prof. Dr. Edson dos Santos Moreira pelas valiosas sugestões e comentários quando da realização do meu exame de qualificação.

Aos Profs. Dr. Luiz Milan e Dr. Heleno Bolfarine pelas valiosas sugestões e comentários quando da realização da defesa da minha Dissertação.

Ao Prof. Dr. Josemar Rodrigues pelo carinho e incentivo demonstrado durante as aulas e nas conversas informais.

À amiga Vera Lúcia Damasceno pelas valiosas sugestões durante as longas e árduas horas de estudo.

Aos professores, funcionários, colegas de pós-gradução e a todos aqueles que de alguma maneira contribuíram para a realização deste trabalho, e em especial aos amigos Adriana, Carlos, Celso, Elisa, Gaby, Josmar, Lilian, Mazé, Pérsida, Ricardo, Rosana, Sadao, Vitória, pela amizade, carinho e apoio durante este periodo, dentro e fora do ICMSC.

Especialmente à minha esposa Elza pelo amor, paciência e incentivo. 


\section{Resumo}

O propósito deste trabalho é investigar uma transformação adequada para a confiabilidade de sistemas visando melhorar a confiabilidade quando consideramos as aproximações normais usuais. Neste sentido, exploramos as reparametrizações de Guerrero e Johnson (1982) e de Aranda-Ordaz (1981), procurando melhorar a aproximação normal da função de verossimilhança (ver Sprott, 1973 ou Anscombe, 1964).

Uma vez consideradas as reparametrizações acima, utilizamos as medidas de normalidade introduzidas por Kass e Slate (1992) a fim de "assegurar" a normalidade nas transformações obtidas e, para avaliar estas transformações, utilizamos um método gráfico baseado no T-plot de Hills e Smith (1993).

Como aplicação, consideramos os modelos exponencial e de Weibull para a confiabilidade de sistemas com uma unidade e desenvolvemos, ainda, uma aplicação com testes acelerados considerando o modelo de lei de potência inversa com tempos de vida com distribuição de Weibull.

Sob o enfoque Bayesiano, exploramos as mesmas técnicas para melhorar a normalidade assintótica das densidades a posteriori de interesse.

Finalizamos considerando 3 exemplos práticos com os modelos exponencial, de Weibull e testes acelerados, onde avaliamos os resultados obtidos com as tranformações propostas. 


\section{Abstract}

The main goal of this work, is to search a transformation of system reliability to improve the usual asymptotical normality results. In this way, we explore the reparametrizations of Guerrero and Johnson (1982) and of Aranda-Ordaz (1981), looking for better normality of the likelihood function (see Sprott, 1973 or Anscombe, 1964). We also use some normality measures for the likelihood function introduced by Kass and Slate (1992) to be used with the above parametrizations and a diagnostic procedure based on the T-plot, introduced by Hills and Smith (1993).

As applications we consider the exponential and weibull models for the system reliability with one unit, and we also consider accelerated life tests with the power rule model and the Weibull distribution.

Under the Bayesian approach, we also consider the same procedure to improve the normality of the posterior densities of interest.

As numerical illustration we present three examples showing the feasibility of the proposed method. 


\section{Índice}

\section{Capítulo 1}

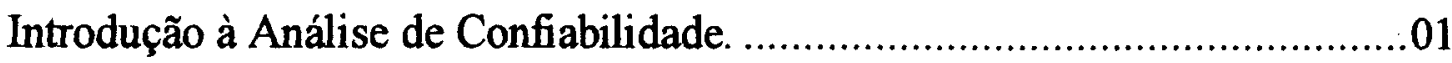

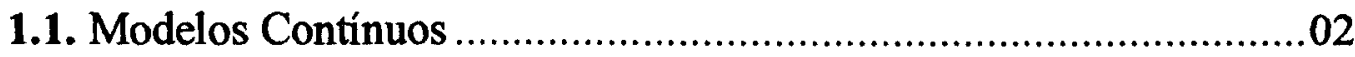

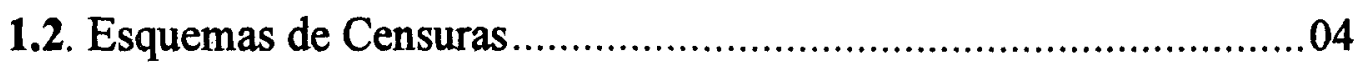

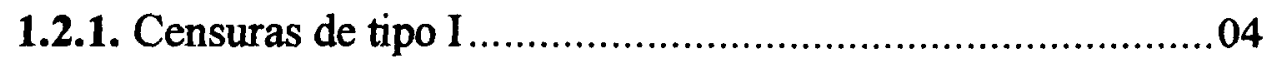

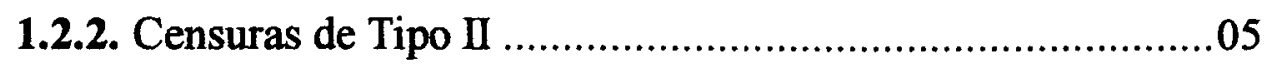

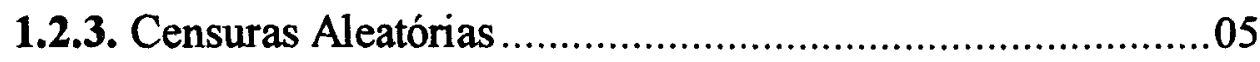

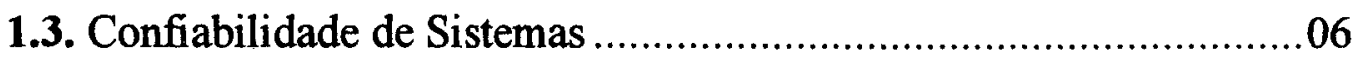

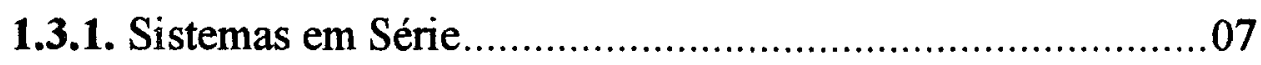

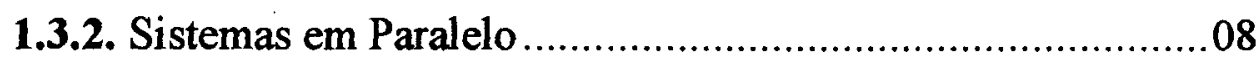

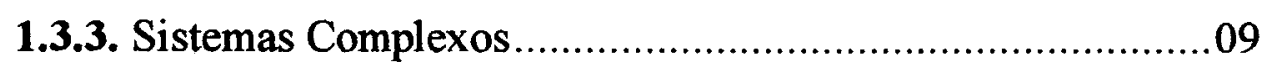

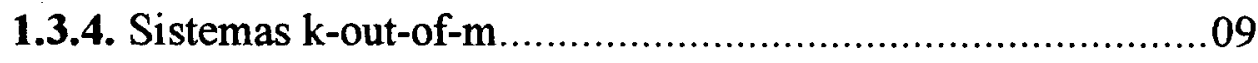

1.4. Objetivos Principais da Pesquisa ................................................12

\section{Capítulo 2}

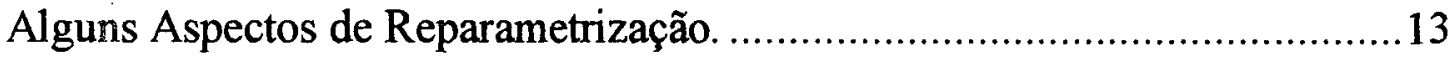

2.1. Algumas Reparametrizações para Proporções ...............................15

2.1.1. Reparametrização de Guerrero e Johnson ...........................15

2.1.2. Reparametrização de Aranda-Ordaz....................................16

2.2. Medidas e Diagnósticos para a Normalidade ................................17

2.2.1. T-Plot de Hills e Smith ......................................................18

2.2.2. Terceira Derivada Padronizada do Logaritmo da

Verossimilhança

\section{Capítulo 3}

Reparametrizações para a Confiabilidade de Sistemas Considerando a Distribuição Exponencial e Dados Censurados.

3.1. Inferências para a Distribuição Exponencial.....................................22

3.1.1. Amostras Completas (sem Censuras) .................................23

3.1.2. Amostras com Dados Censurados ........................................24 
3.2. Estimação da Confiabilidade de Sistemas com uma Unidade

3.2.1. Reparametrização de Guerrero e Johnson para a

Confiabilidade .28

3.2.2. Reparametrização de Aranda-Ordaz para a

Confiabilidade 30

\section{Capítulo 4}

Estimação Bayesiana para a Confiabilidade de Sistemas com uma Unidade Considerando a Distribuição Exponencial

4.1. Fórmula de Bayes 34

4.2. Densidades a Priori e a Posteriori. 34

4.3. Análise Bayesiana para a Confiabilidade de Sistemas

Considerando uma Densidade a Priori Não-Informativa de Jeffreys e Dados Censurados . 36

4.4. Análise Bayesiana para a Confiabilidade de Sistemas

Considerando uma Densidade a Priori Beta e Dados Censurados ...40

\section{Capítulo 5}

Reparametrização para a Confiabilidade Considerando o Modelo de Weibull e Dados Censurados.

5.1. Inferências para a Distribuição de Weibull .43

5.1.1. Amostras Completas .43

5.1.2. Amostras Censuradas. .45

5.2. Estimação da Confiabilidade de Sistemas com uma Unidade .46

5.2.1. Inferências para o Modelo de Weibull com $\beta$ Conhecido....48

5.2.2. Reparametrização de Guerreiro de Johnson para a

Confiabilidade $\operatorname{com} \beta$ Conhecido. .50

5.2.3. Inferências para o Modelo de Weibull com $\beta$ Desconhecido

5.3. Análise Bayesiana.

5.3.1. Reparametrização de Guerreiro de Johnson para a

Densidade a Posteriori. 57

5.3.2. Análise Bayesiana com $\beta$ Conhecido .57 


\section{Capítulo 6}

Reparametrização em Testes Acelerados Considerando o Modelo de

Lei de Potência Inversa.

6.1. Inferências para a Confiabilidade de Sistemas com uma Unidade...60

6.1.1. Estimadores de Máxima Verossimilhança .63

6.2. Caso Especial $\beta=1$ : Distribuição Exponencial...............................65

6.2.1. Reparametrização de Guerrero e Johnson com $\beta=1$. .67

6.2.2. Inferências para a Confiabilidade Considerando a Função de Verossimilhança Profile. 68

6.2.3. Reparametrização de Guerrero e Johınson para a Função de Verossiilhança Profile. .70

6.2.4. Inferências para a Confiabilidade com $\beta=1$ e $\gamma$ Conhecido

6.2.5. Reparametrização de Guerrero e Johnson para a Função de Confiabilidade com $\beta=1$ e $\gamma$ Conhecido .73

6.3. Análise Bayesiana Considerando $\beta=1$ e $\gamma$ Desconhecido .75

6.3.1. Reparametrização de Guerrero e Johnson para a Densidade a Posteriori .77

6.3.2. Análise Bayesiana com $\beta=1$ e $\gamma$ Conhecido .77

\section{Capítulo 7}

Exemplos 79

7.1. Exemplo para um Sistema com uma Unidade, Considerando o Modelo Exponencial.

7.2. Exemplo para um Sistema com uma Unidade, Considerando o Modelo de Weibull. .91

7.3. Exemplo com Testes Acelerados Considerando o Modelo de Lei de Potência Inversa. .101 
Apêndice A.

Derivadas do Logaritmo da Função de Verossimilhança $l\left(\phi_{G J}\right)$ Localmente no Estimador de Máxima Verossimilhança $\hat{\phi}_{G J}(\lambda)$.

Apêndice B.

Derivadas do Logaritmo da Função de Verossimilhança $l\left(\phi_{A O}\right)$ Localmente no Estimador de Máxima Verossimilhança $\hat{\phi}_{A O}(\lambda)$

Apêndice C.

O Método Delta

Apêndice D.

O Método de Laplace para Aproximação de Integrais

Apêndice E

Programas Desenvolvidos. 


\section{Capítulo 1}

\section{INTRODUÇÃO À ANÁLISE DE CONFIABILIDADE.}

Muitas técnicas estatisticas têm sido aplicadas em engenharia, especialmente para o estudo do tempo de vida de unidades (uma unidade pode ser uma peça ou componente, um sistema de componentes, ou mesmo um equipamento). Também podemos aplicar as mesmas técnicas em medicina, por exemplo, na análise do tempo de sobrevivência de um paciente até sua morte ou recuperação. As aplicações em engenharia são usualmente conhecidas como análise de Confiabilidade, enquanto que na medicina como análise de Sobrevivência. Encontramos na literatura estatística muitas técnicas apropriadas para análises deste tipo (ver Lawless, 1982; Miller, 1981; Kalbfleish e Prentice, 1980; ou Martz e Waller ,1982).

Geralmente, na engenharia são utilizadas técnicas paramétricas com modelos contínuos, pois na maioria dos casos os dados possuem informação suficiente para a identificação de um modelo; além disso, as técnicas paramétricas são mais eficientes que as não-paramétricas. Nosso trabalho será baseado em modelos paramétricos contínuos, portanto serão estes modelos que consideraremos a partir de agora ${ }^{1}$.

1 Para informaç̃̃es sobre modelos não paramétricos, ver Lawless, 1982 ou Miller, 1981. 


\subsection{Modelos Contínuos.}

Seja $T$ uma variável aleatória não negativa representando 0 tempo de funcionamento de um componente. Considerando $f(t)$ a função de densidade de probabilidade de $T$, então a função de distribuição e a função de confiabilidade de $T$ são, respectivamente:

$$
F(t)=P(T \leq t)=\int_{0}^{t} f(x) d x
$$

e

$$
R(t)=P(T>t)=\int_{t}^{\infty} f(x) d x
$$

A função de Risco ${ }^{2}$ de $T$ é definida por:

$$
h(t)=\lim _{\Delta t \rightarrow 0_{+}} \frac{P[t \leq T<t+\Delta t / T \geq t]}{\Delta t}=\frac{f(t)}{R(t)}
$$

Observar que também podemos definir a função de risco por:

$$
h(t)=-\frac{d \ln [R(t)]}{d t}
$$

A função de risco acumulado é dada por:

$$
H(t)=\int_{0}^{t} h(x) d x
$$

2 A função de risco especifica a taxa instantânea de falha em $T=t$, condicionada à sobrevivência até o instante $t$. 
Segundo as definições (1.4) e (1.5), temos as seguintes relações:

$$
\begin{aligned}
& R(t)=\exp \left\{-\int_{0}^{t} h(x) d x\right\}, \quad \text { pois } R(0)=1 \\
& R(t)=\mathrm{e}^{-H(t)}
\end{aligned}
$$

Desta forma, de (1.3), a função de densidade de $T$ pode ser representada por:

$$
f(t)=h(t) \exp \left\{-\int_{0}^{t} h(x) d x\right\}
$$

EXEMPLO 1: Seja uma v.a. $T$ com distribuição Exponencial com parâmetro $\theta$, cuja função densidade é dada por:

$$
f(t)=\frac{1}{\theta} \mathrm{e}^{-t / \theta}, \quad t>0, \theta>0 .
$$

A média e variância de $T$ são $E(T)=\theta$ e $\operatorname{Var}(T)=\theta^{2}$.

As funções de risco e de confiabilidade são dadas, respectivamente, por:

$$
h(t)=\frac{1}{\theta} \quad \text { e } \quad R(t)=\mathrm{e}^{-t / \theta}
$$

Outros modelos podem ser considerados, tais como o de Weibull, Valor Extremo, Gama, Log-Normal, etc... 


\subsection{Esquemas de Censuras.}

Nos estudos de confiabilidade são frequentes amostras onde, por questão de custo ou de tempo, não observamos os tempos de vida de algumas unidades. Quando isso ocorre, dizemos que os tempos de vida dessas unidades são censurados.

Esta situação ocorre, por exemplo, quando uma amostra é colocada em teste para estudo do tempo de vida de seus componentes, e, devido ao alto custo, o experimento é interrompido após a ocorrência de um certo número de falhas $d$ pré-fixado (uma falha ocorre quando o componente deixa de operar satisfatoriamente). Um outro exemplo acontece quando o experimento é interrompido após um certo tempo $L$, sendo que vários componentes ainda continuam em funcionamento.

É importante que consideremos estas unidades com censuras em nossas análises para não ocorrermos em inferências viciadas ou menos eficientes.

\subsubsection{Censuras de Tipo $I$.}

Uma censura do tipo I ocorre quando temos experimentos com um período fixo de observação. Uma possibilidade muito comum em engenharia, consiste em considerarmos $n$ unidades em teste e encerrarmos o experimento após um tempo fixo $L$. Outra situação, mais comum na medicina, consiste em um experimento onde nem todas as unidades iniciam o teste no mesmo instante, sendo que cada unidade tem seu tempo de censura $L_{i}, i=1, \ldots, n$, fixo. Desta forma, os componentes cujas falhas ocorreram antes do término do experimento, terão seus tempos de vida observados e, os que continuarem em funcionamento, serão considerados censurados. $\mathrm{Na}$ figura 1 apresentamos um esquema de censuras do tipo I. 


\subsubsection{Censuras de Tipo II.}

Uma amostra com censuras de tipo II é aquela onde apenas as primeiras $d$ falhas, numa amostra aleatória de tamanho $n$, são observadas ( $d$ fixo, $1 \leq d \leq n$ ). Censuras de tipo II geralmente ocorrem em ensaios de tempos de vida; uma amostra com $n$ componentes é colocada em teste e o experimento termina após a ocorrência da $d$ ésima falha. Estes ensaios economizam tempo e custo, uma vez que poderíamos ter um período muito longo de teste para a observação de todas as falhas.

$\mathrm{Na}$ figura 2 apresentamos um diagrama com o esquema de censuras de tipo II.

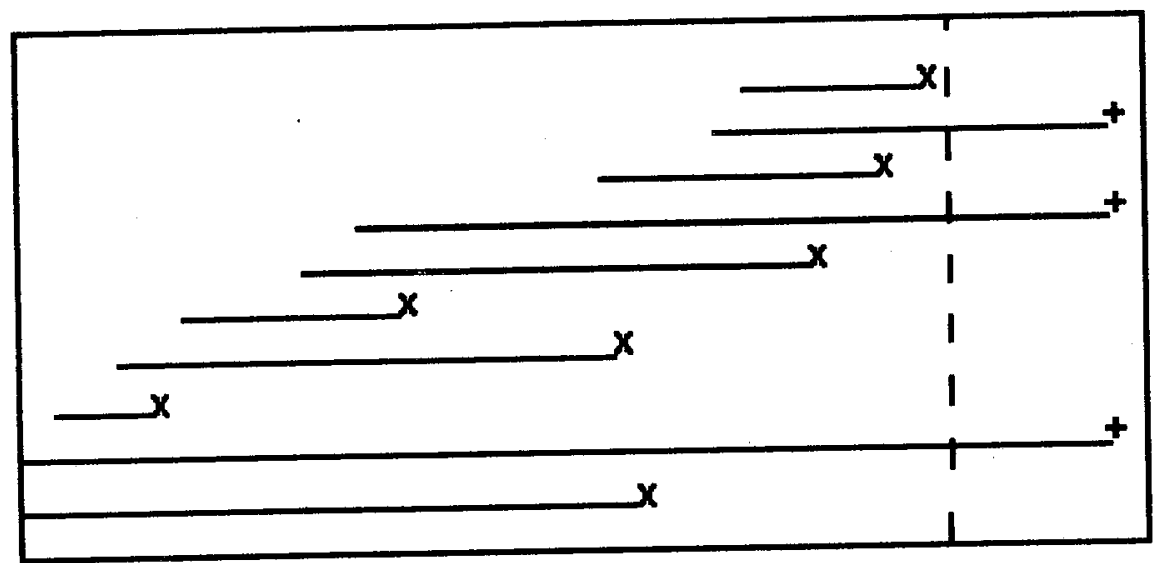

Figura 1: Esquema de Censuras de Tipo I

$$
X=\text { falha } \quad+=\text { censura. }
$$

\subsubsection{Censuras Aleatórias.}

Em algumas aplicações, especialmente na área médica, os tempos censurados, são aleatórios; por exemplo, em um experimento médico os pacientes podem entrar em estudo de uma forma aleatória, de acordo com a data do diagnóstico. Se o estudo 
terminar numa data pré-estabelecida, então, os tempos de censuras, que são os intervalos entre o instante que o indivíduo entra em estudo até o término do mesmo, são aleatórios. Frequentemente, nestes casos, consideramos censuras do tipo I, assumindo dados condicionais nos tempos de censuras observados.

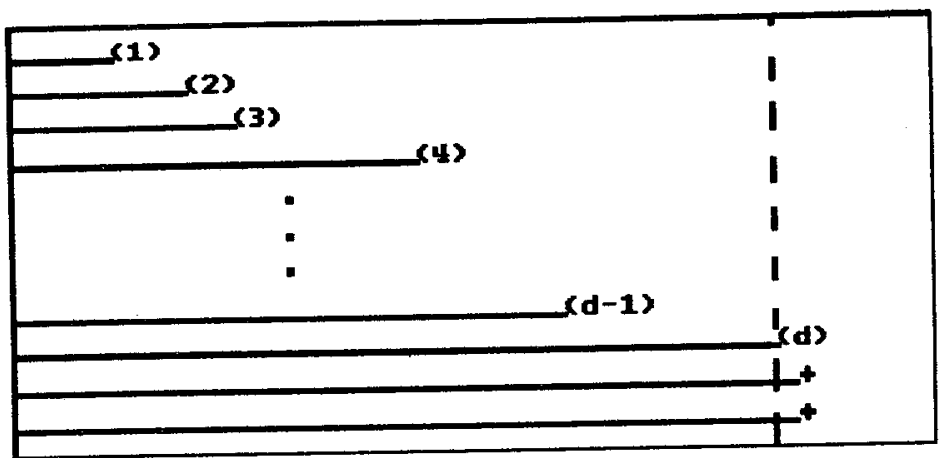

Figura 2: Esquema de censuras de tipo II.

$$
(.)=\text { falha } \quad+=\text { censura }
$$

\subsection{Confiabilidade de Sistemas.}

Uma aplicação muito importante da teoria de confiabilidade diz respeito à análise de confiabilidade de sistemas. Um sistema é definido por um grupo de subsistemas ou componentes, cujo funcionamento adequado determina se o sistema irá operar satisfatoriamente.

Existem basicamente três tipos de sistemas: sistemas em paralelo, em série e sistemas complexos. Uma representação bastante funcional para indicar o efeito da falha de um subsistema ${ }^{3}$ sobre o desempenho do sistema global, é o diagrama de blocos, conforme figuras 3 e 4 . Para o tratamento estatístico, consideraremos todos os

3 Um subsistema poderá ser a designação dada a um particular grupamento de componentes ou a um componente apenas. 
subsistemas independentes, ou seja, o conhecimento do tempo de falha de um subsistema não afetará a distribuição do tempo de falha dos demais. Também, por questão de simplificação, daqui em diante consideraremos cada subsistema simplesmente como um componente.

\subsubsection{Sistemas em Série.}

A configuração em série é a situação mais comum de se encontrar, e é também, a mais simples para se analisar. Um sistema em série funciona se todos os seus componentes funcionarem simultâneamente. $O$ diagrama de blocos para um sistema em série é apresentado pela figura 3 .

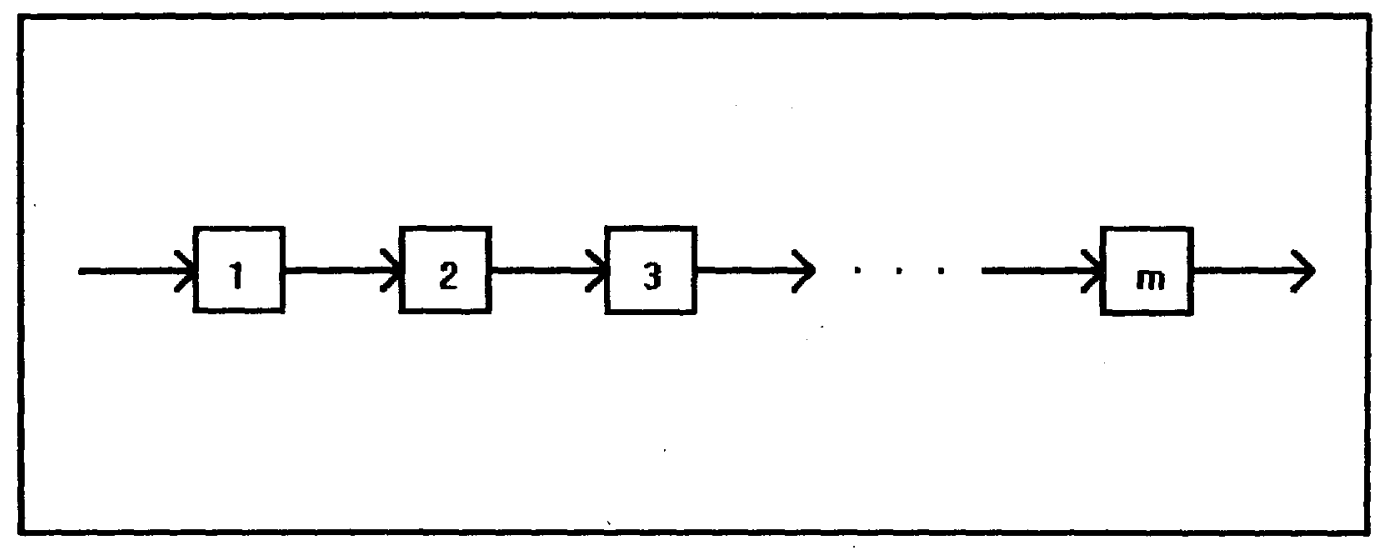

Figura 3: Diagrama de blocos para sistema em série.

A confiabilidade de um sistema em série é dada por: 


$$
\begin{aligned}
R_{S}(t) & =P\left(T_{S} \geq t\right)=P\left(T_{1} \geq t\right) \cdot P\left(T_{2} \geq t\right) \quad \cdots \quad P\left(T_{m} \geq t\right) \\
& =\prod_{i=1}^{m} R_{i}(t) .
\end{aligned}
$$

onde: $\left\{\begin{array}{l}T_{S}=\text { tempo de falha do sistema } \\ T_{i}=\text { tempo de falha do } i \text { - ésimo componente. }\end{array}\right.$

\subsubsection{Sistemas em Paralelo.}

Um sistema em paralelo é mostrado pela figura 4. O sistema opera satisfatoriamente se pelo menos $k$ ou mais componentes funcionarem $(k \leq n)$. Se $k=1$ o sistema é chamado sistema paralelo puro e, se $k>1$, é chamado sistema $k$-out-of-m.

A confiabilidade para um sistema em paralelo puro $(k=1)$ é definida por:

$$
\begin{aligned}
R_{P}(t) & =P\left(T_{P} \geq t\right)=1-P\left(T_{P} \leq t\right)=1-P\left(T_{1} \leq t, T_{2} \leq t, \ldots, T_{m} \leq t\right) \\
& =1-\prod_{i=1}^{m} F_{i}(t)=1-\prod_{i=1}^{m}\left[1-R_{i}(t)\right] .
\end{aligned}
$$

$$
\text { onde: }\left\{\begin{array}{l}
T_{P}=\text { tempo de falha do sistema } \\
T_{i}=\text { tempo de falha do } i \text { - ésimo componente. }
\end{array}\right.
$$




\subsubsection{Sistemas Complexos.}

Um sistema que não é em série nem em paralelo puro (ou uma combinação dos dois) é chamado sistema complexo. A determinação da confiabilidade de um sistema complexo não é tão simples como nos casos anteriores. Shooman (1968) descreve diversos métodos que funcionam satisfatoriamente bem quando o número de falhas que interrompe o sistema é pequeno.

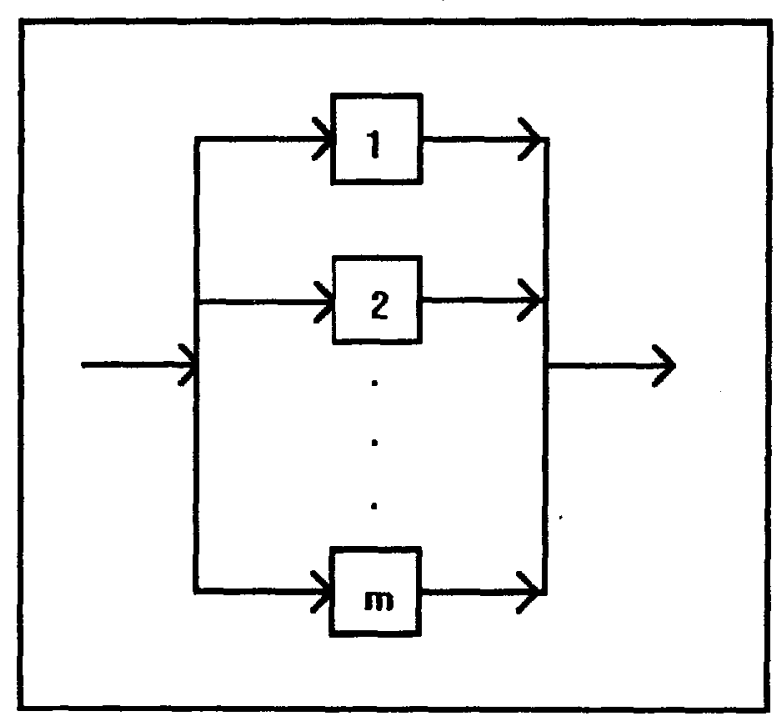

Figura 4: Diagrama de blocos para um sistema em paralelo puro.

\subsubsection{Sistemas $k$-out-of- $m$.}

Supor que $\mathbf{S}$ é um sistema em paralelo com $\boldsymbol{m}$ componentes tal que o sistema funciona se e só se pelo menos $k$ componentes operam satisfatoriamente. Este sistema 


$$
\begin{aligned}
R(t) & =P(T>t) \\
& =\sum_{j=k}^{m}\left(\begin{array}{c}
m \\
j
\end{array}\right)[1-F(t)]^{j}[F(t)]^{m-j}
\end{aligned}
$$

Uma das distribuições mais utilizadas para a confiabilidade de sistemas é dada pela distribuição exponencial para o tempo de vida dos componentes, com densidade (1.9). Com essa distribuição, a confiabilidade de um sistema $k$-out-of- $m$, num tempo $t$, é dada por:

$$
R(t)=\sum_{j=k}^{m} \sum_{l=0}^{m-j}(-1)^{l}\left(\begin{array}{c}
m \\
j
\end{array}\right)\left(\begin{array}{c}
m-j \\
l
\end{array}\right) \exp \left\{-\frac{t(j+l)}{\theta}\right\}
$$

EXEMPLO 2: Considere um sistema com $m=3$ componentes e que opera satisfatoriamente se e só se pelo menos $k=2$ componentes funcionarem. Esse sistema é denominado 2-out-of-3. Assumindo que os tempos de vida dos componentes são independentes, exponencialmente distribuidos segundo a densidade (1.9), então a função de confiabilidade obtida de (1.14) é dada por:

$$
R(t)=\sum_{j=2}^{3} \sum_{l=0}^{3-j}(-1)^{l}\left(\begin{array}{c}
3 \\
j
\end{array}\right)\left(\begin{array}{c}
3-j \\
l
\end{array}\right) \exp \left\{-\frac{t(l+j)}{\theta}\right\}=3 \mathrm{e}^{-2 t / \theta}-2 \mathrm{e}^{-3 t / \theta}
$$

Expandindo em Taylor, temos: 
Expandindo em Taylor, temos:

$$
\left\{\begin{array}{l}
\mathrm{e}^{-2 t / \theta} \approx 1-\frac{2 t}{\theta}+\frac{2 t^{2}}{\theta^{2}} \\
\mathrm{e}^{-3 t / \theta} \approx 1-\frac{3 t}{\theta}+\frac{9 t^{2}}{2 \theta^{2}}
\end{array}\right.
$$

Portanto, a função de confiabilidade dada em (1.15) pode ser aproximada por:

$$
R(t) \approx 1-\frac{3 t^{2}}{\theta^{2}}
$$

Com o modelo exponencial e usando métodos clássicos de inferência, Pugh (1963) e Basu (1964) encontraram o estimador não-viciado de variância mínima (UMVUE) para a confiabilidade de sistemas com uma unidade. Para sistemas em série ou em paralelo, Rutemiller (1966) encontrou estimadores pontuais para a confiabilidade e Basu e El Mawaziny (1978) obtiveram UMVUE e estimadores de máxima verossimilhança (EMV) nos casos gerais de sistemas $k$-out-of-m. Zacks e Even (1966) estudaram a eficiência relativa dos estimadores para sistemas com uma unidade e para amostras pequenas, e Chao (1981) encontrou fórmulas aproximadas para os erros quadráticos médios dos estimadores da confiabilidade para sistemas $k$-out-of- $m$ no caso de componentes independentes e exponencialmente distribuidos. Métodos Bayesianos para a estimativa da função de confiabilidade de sistemas $k$-out-of- $m$ também têm sido apresentados de forma extensiva na literatura (ver por exemplo Martz e Waller, 1982). 


\subsection{Objetivos Principais da Pesquisa.}

O projeto de pesquisa apresentado neste trabalho tem como objetivo principal explorar alguns aspectos de reparametrizações para melhorar as inferências sobre a confiabilidade $R(t)$ de sistemas num dado instante $t$, especialmente para amostras pequenas e moderadas. Com as possíveis reparametrizações para $R(t)$, pretendemos avaliar a precisão das inferências obtidas. Os principais pontos são:

i) Inicialmente consideraremos a distribuição exponencial para os tempos de vida dos componentes, explorando também outros modelos em confiabilidade como a distribuição de Weibull;

ii) Pretendemos considerar dados com censuras, e também utilizar métodos Bayesianos com diferentes distribuições a priori para o parâmetro de interesse (por exemplo o uso da densidade a priori de Jeffreys; ver Box e Tiao 1973);

iii) Na nova parametrização, quando não for possível a obtenção de uma solução analítica, usaremos métodos numéricos ou de aproximação na obtenção dos momentos e densidades a posteriori para a confiabilidade;

iv) Também pretendemos avaliar as inferências obtidas, considerando as aproximações normais usuais para o estimador de máxima verossimilhança de $R(t)$, comparando-as com as obtidas na nova reparametrização.

Esses resultados poderão ser de grande interesse prático, especialmente na área industrial envolvendo componentes eletrônicos. 


\section{Capítulo 2}

\section{ALGUNS ASPECTOS DE REPARAMETRIZAÇÃO.}

É prática freqüente entre os estatísticos e pesquisadores fazer inferências sobre funções de parâmetros, como por exemplo, a confiabilidade num dado instante $t_{0}$. Geralmente nessas situações, não é possível se obter resultados exatos, sendo comum considerar-se a Normalidade Assintótica do Estimador de Máxima Verossimilhança, baseada na informação de Fisher ou informação observada (ver por exemplo Bickel e Doksum, 1977).

Esses resultados assintóticos podem não ser apropriados quando trabalhamos com amostras pequenas ou moderadas, principalmente quando estamos interessados numa confiabilidade muito baixa $(R \approx 0)$ ou muito alta $(R \approx 1)$. A situação é ainda mais crítica quando trabalhamos com dados censurados, o que é muito freqüente com experimentos de tempos de vida.

Uma alternativa para melhorar estes resultados é considerar reparametrizações que melhorem a normalidade da função de verossimilhança (ver por exemplo, Anscombe, 1964 ou Sprott, 1973).

Em inferência Bayesiana, também é importante uma boa parametrização para se obter resultados mais precisos quando utilizamos métodos numéricos ou de aproximação no cálculo de momentos a posteriori (ver por exemplo, Achcar e Smith, 1990; Kass e Slate, 1992 ou Hills e Smith, 1993). 
Como já citamos anteriormente, uma maneira de melhorar a normalidade do estimador de máxima verossimilhança para a função de confiabilidade num dado instante $t_{0}$, é considerar diferentes parametrizações, ou transformações, de $R\left(t_{0}\right)$. Uma transformação possível neste caso é a transformação logito $\phi_{L}=\ln [R /(1-R)]$, onde $R=R\left(t_{0}\right)$.

Várias famílias de transformações para proporções são sugeridas na literatura (ver por exemplo Atkinson, 1985) as quais podem ser utilizadas para melhorar a normalidade da função de verossimilhança de $R\left(t_{0}\right)$. Uma destas transformações, que produz a transformação logito quando $\lambda=0$, é a transformação "folded power" (ver Mosteller e Tukey, 1977), dada por:

$$
\phi_{F}(\lambda)=R^{\lambda}-(1-R)^{\lambda}, \quad 0 \leq R \leq 1 .
$$

Esta transformação tem a desvantagem de não possuir inversa, exceto para os casos especiais de $\lambda$ iguais a 0 ou 1 .

Em nosso trabalho exploraremos duas transformações introduzidas por Guerrero e Johnson (1982) e Aranda-Ordaz (1981), as quais foram propostas originalmente para análise de dados binários, mas que podem ser facilmente adaptadas para análise de confiabilidade. 


\subsection{Algumas Reparametrizações para Proporções.}

\subsubsection{Reparametrização de Guerrero e Johnson.}

Para obter uma família de transformações inversíveis que inclui a transformação logito, Guerrero e Johnson (1982) sugeriram uma aplicação da transformação potência de Box e Cox (1964) para a "odds ratio", $R /(1-R)$, dada por:

$$
\phi_{G J}^{*}(\lambda)=\left[\left(\frac{R}{1-R}\right)^{\lambda}-1\right] / \lambda, \quad-1 \leq \phi_{G J}(\lambda)<\infty
$$

onde $R=R\left(t_{0}\right)$.

Para $\lambda=0$ temos a transformação logito e para um dado $\lambda$, podemos considerar uma forma modificada da transformação de Guerrero e Johnson dada por:

$$
\phi_{G J}(\lambda)=\left(\frac{R}{1-R}\right)^{\lambda}-1
$$

que não produz resultados diferentes dos obtidos com (2.2), (ver Atkinson, 1985).

A vantagem da transformação sugerida por Guerrero e Johnson é que ela é facilmente invertida. Fazendo $\phi_{G J}=\phi_{G J}(\lambda)$, temos:

$$
R\left(t_{0}\right)=\frac{\left(1+\phi_{G J}\right)^{1 / \lambda}}{1+\left(1+\phi_{G J}\right)^{1 / \lambda}}
$$


Desta forma, a transformação pode ser facilmente usada para se obter estimativas e intervalos de confiança para os parâmetros com os dados na escala original.

\subsubsection{Reparametrização de Aranda-Ordaz.}

Outra transformação para proporções sugerida por Aranda-Ordaz (1981) é dada por:

$$
\phi_{A O}(\lambda)=\frac{R^{\lambda}-(1-R)^{\lambda}}{R^{\lambda}+(1-R)^{\lambda}}
$$

isto é,

$$
\phi_{A O}(\lambda)=\frac{1-\left(R^{-1}-1\right)^{\lambda}}{1+\left(R^{-1}-1\right)^{\lambda}}
$$

$\operatorname{com}-1 \leq \phi_{A O}(\lambda) \leq 1, \quad 0 \leq R \leq 1$

Note que em (2.5) e (2.6) temos a transformação logito quando $\lambda=0$ e a transformação identidade quando $\lambda=1$.

Novamente temos uma transformação facilmente inversível: fazendo $\phi_{A O}=\phi_{A O}(\lambda)$, temos

$$
R\left(t_{0}\right)=\left[\left(\frac{1-\phi_{A O}}{1+\phi_{A O}}\right)^{1 / \lambda}+1\right]^{-1}
$$


Assim, com esta transformação, também podemos facilmente obter estimativas e intervalos de confiança para os parâmetros com os dados na escala original.

\subsection{Medidas e Diagnósticos para a Normalidade.}

Como já foi citado, ao adotarmos a normalidade assintótica para o estimador de máxima verossimilhança podemos estar assumindo uma aproximação que pode não condizer com a realidade, por isso é importante que possamos verificar essa suposta normalidade. Um exemplo dessa situação é dada quando estamos interessados em valores extremos da confiabilidade $\left[R\left(t_{0}\right) \approx 0\right]$; nesses casos observamos uma assimetria muito acentuada para a função de verossimilhança. Quando utilizamos uma reparametrização, é importante verificar o quanto conseguimos melhorar a normalidade da função de verossimilhança e em análise Bayesiana por sua vez, é importante avaliar a normalidade das distribuições a posteriori.

Um modo muito simples para se verificar a normalidade da função de verossimilhança, ou de densidades a posteriori de interesse, é através de gráficos e contornos no caso bivariado. No caso bivariado, por exemplo, conclúmos pela normalidade quando encontramos contornos com formas de elipses aproximadas.

Em situações quando temos interesse num dado parâmetro e existem vários parâmetros auxiliares (nuisance parameters), podemos como uma alternativa, considerar a verossimilhança "profile" 4 e verificar sua normalidade através de gráficos.

\footnotetext{
${ }^{4}$ A função de verosimilhança "profile" para o parâmetro $\theta_{j}$ é definida por: $L\left(\theta_{j}\right)=\max _{\theta_{1}, \cdots, \theta_{j-1}, \theta_{j+1}, \cdots, \theta_{p}} L(\theta)$.
} 


\subsubsection{T-Plot de Hills e Smith.}

Um método gráfico bastante prático para se verificar a normalidade é dado pelo T-Plot introduzido por Hills e Smith (1993).

Em geral, ao fazermos uma análise gráfica da função de verossimilhança, ficamos restritos a avaliar apenas a simetria ou elipticidade dos gráficos, não tendo como medir o seu "grau" de normalidade, ou seja, o quanto estamos próximos ou não da normalidade desejada.

Hills e Smith introduziram o método T-Plot como generalização e extensão a um método proposto por de Bates e Watts (1980) onde a normalidade pode ser avaliada através da linearidade dos gráficos.

Seja $p(\hat{\theta} /$ dados $)$ o valor da densidade a posteriori calculada na moda $\hat{\theta}=\left(\hat{\theta}_{1}, \hat{\theta}_{2}, \ldots, \hat{\theta}_{p}\right)$, e seja $p\left(\theta_{j} /\right.$ dados $)$ a densidade a posteriori "profile" de $\theta_{j}$, dada por:

$$
p\left(\theta_{j} / \text { dados }\right)=\max _{\theta_{1}, \cdots, \theta_{j-1}, \theta_{j+1}, \cdots, \theta_{p}} p(\theta / \text { dados })
$$

Uma medida de diagnóstico é dada por,

$$
T\left(\theta_{j}\right)=\operatorname{sgn}\left(\theta_{j}-\hat{\theta}_{j}\right)\left\{-2 \ln \left[\frac{p\left(\theta_{j} / \text { dados }\right)}{p(\hat{\theta} / \text { dados })}\right]\right\}^{1 / 2},
$$

onde $\operatorname{sgn}(x)=x /|x|$ é a função sinal de $x$. 
O gráfico de $T\left(\theta_{j}\right)$ versus $\theta_{j}$ deverá ser uma linha reta caso o parâmetro $\theta_{j}$ seja normalmente distribuído; conseqüentemente, o gráfico pode ser usado para identificar desvios da normalidade.

No caso unidimensional o T-plot de Hills e Smith é definido (de 2.9), por,

$$
T(\theta)=\operatorname{sgn}(\theta-\hat{\theta})\left\{-2 \ln \left[\frac{p(\theta / \text { dados })}{p(\hat{\theta} / \text { dados })}\right]\right\}^{1 / 2} .
$$

O caso bidimensional é mais complexo, pois a extensão óbvia de (2.10) não funciona. Nessa situação aplicamos a expansão de Taylor para $\log \left[p\left(\theta_{1}, \theta_{2} /\right.\right.$ dados $\left.)\right]$ em torno de $\hat{\theta}=\left(\hat{\theta}_{1}, \hat{\theta}_{2}\right)$, a moda de $p\left(\theta_{1}, \theta_{2} /\right.$ dados $)$. Desta forma, procedendo como no caso anterior, construímos o contorno de $T\left(\theta_{1}, \theta_{2}\right)$ versus $\theta_{1}$ e $\theta_{2}$. Se $\left(\theta_{1}, \theta_{2}\right)$ são normalmente distribuídos, então o contorno deverá ter forma elíptica. Entretanto, temos aqui dois problemas: é mais dificil julgar "grau de elipticidade" do que "grau de linearidade" e podemos simplesmente construir contornos para a densidade a posteriori $p\left(\theta_{1}, \theta_{2} /\right.$ dados $)$ e analisá-los diretamente. Uma solução, então, seria fixarmos $\theta_{2}$ e analisarmos o comportamento de $\theta_{1}$ isoladamente.

Em nosso trabalho utilizaremos o T-Plot de Hills e Smith para a função de verossimilhança $L(\theta)$, a partir da seguinte relação:

$$
\begin{aligned}
T(\theta) & =\operatorname{sgn}(\theta-\hat{\theta})\left\{-2 \ln \left[\frac{L(\theta)}{L(\hat{\theta})}\right]\right\}^{1 / 2} \\
& =\operatorname{sgn}(\theta-\hat{\theta})\{-2[l(\theta)-l(\hat{\theta})]\}^{1 / 2} .
\end{aligned}
$$


onde $l(\theta)$ é o logaritmo da função de verossimilhança.

\subsubsection{Terceira Derivada Padronizada do Logaritmo da Verossimilhança.}

Kass e Slate (1992) propõem uma medida de não-normalidade de densidades a posteriori ou função de verossimilhança, dada pela terceira derivada padronizada (STD), que simplifica as interpretações pois reduz as análises a um simples escalar. A terceira derivada padronizada do logaritmo da verossimilhança foi originalmente proposta por Sprott (1973) e é dada por:

$$
S T D(\hat{\theta})=\left|l^{\prime \prime \prime}(\hat{\theta}) \cdot\left[-l^{\prime \prime}(\hat{\theta})\right]^{-3 / 2}\right|
$$

onde $l^{\prime \prime}(\hat{\theta})$ e $l^{\prime \prime \prime}(\hat{\theta})$ são, respectivamente, a segunda e tèrceira derivadas do logaritmo da função de verossimilhança calculado no estimador de máxima verossimilhança $\hat{\theta}$. Se a função de verossimilhança apresentar "boa normalidade", então esperamos encontrar $\operatorname{STD}(\hat{\theta})$, dado em (2.12), próximo ou igual a zero. De forma análoga, podemos calcular a terceira derivada padronizada para a densidade a posteriori $p(\theta /$ dados $)$ substituindo o estimador de máxima verossimilhança $\hat{\theta}$ pela sua moda $\tilde{\theta}$.

No caso multiparamétrico uma generalização da terceira derivada padronizada $(S T D)$, é dada pela medida global de não-normalidade de Kass e Slate (1992), definida por:

$$
m^{2} \bar{B}^{2}(\hat{\theta})=\sum_{a, b, c, d, e, f} d_{a b c} d_{d e f} b_{a b} b_{d e} b_{c f},
$$


onde $b_{a b}$ são os elementos da inversa da matriz de informação, $d_{a b c}=\partial^{3} l(\hat{\theta}) / \partial \theta_{a} \partial \theta_{b} \partial \theta_{c}$ e $m$ é o número de parâmetros.

Caso os parâmetros considerados tenham uma boa normalidade conjunta, então esperamos encontrar $m^{2} \bar{B}^{2} \approx 0$. Essa medida reduz-se à (2.12) no caso uniparamétrico.

Neste trabalho, utilizaremos a terceira derivada padronizada do logaritmo da verossimilhança STD e a medida global de não-normalidade $m^{2} \bar{B}^{2}$ para obter um valor : apropriado de $\lambda$ que forneça boa normalidade para as reparametrizações consideradas. Assim sendo, no caso uniparamétrico com uma nova parametrização $\phi(\lambda)$, procuramos $\lambda$ tal que a terceira derivada padronizada do logaritmo da função de verossimilhança no ponto $\hat{\phi}(\lambda)$, dada por:

$$
\operatorname{STD}[\hat{\phi}(\lambda)]=\left|l^{\prime \prime \prime}(\hat{\phi}(\lambda))\left[-l^{\prime \prime}(\hat{\phi}(\lambda))\right]^{-3 / 2}\right|
$$

seja próxima ou igual a zero . 


\section{Capítulo 3}

\section{REPARAMETRIZAÇÕES PARA A CONFIABILIDADE DE SISTEMAS CONSIDERANDO A DISTRIBUIÇÃO EXPONENCIAL E DADOS CENSURADOS.}

Historicamente a distribuição exponencial foi o primeiro modelo para estudos de tempos de vida, ocupando, portanto, uma posição importante nesta área. Metodologias estatísticas têm sido desenvolvidas extensivamente para o modelo exponencial, fornecendo-lhe, assim, uma vasta bibliografia. O modelo exponencial foi o primeiro modelo criado para os estudos de tempos de vida, sendo talvez o mais utilizado em confiabilidade, assim como a distribuição normal em outras áreas da estatística.

Neste capítulo trataremos do problema da utilização da normalidade assintótica do estimador de máxima verossimilhança para a confiabilidade de sistemas, explorando as reparametrizações de Guerrero e Johnson e Aranda-Ordaz, dadas em (2.3) e (2.5), respectivamente.

\subsection{Inferências para a Distribuição Exponencial.}

Seja uma amostra aleatória de tamanho $n$, com tempos de vida $T_{1}, T_{2}, \ldots, T_{n}$, exponencialmente distribuídos com densidade (ver 1.9), dada por 


$$
f\left(t_{i}\right)=\frac{1}{\theta} \mathrm{e}^{-t_{i} / \theta}, \quad t_{i}>0, \theta>0
$$

e função de confiabilidade $R\left(t_{i}\right)=\mathrm{e}^{-t_{i} / \theta}, i=1,2, \ldots, n$.

\subsubsection{Amostras Completas (sem censuras).}

Com dados completos, a função de verossimilhança para o parâmetro $\theta$ da densidade exponencial em (3.1) é dada por:

$$
L(\theta)=\prod_{i=1}^{n} f\left(t_{i} ; \theta\right)=\frac{1}{\theta^{n}} \mathrm{e}^{-T / \theta}, \quad \text { onde } T=\sum_{i=1}^{n} t_{i}
$$

O logaritmo da função de verossimilhança para $\theta$ é dado por:

$$
l(\theta)=\ln [L(\theta)]=-n \ln (\theta)-\frac{T}{\theta}
$$

A estatística $T$ é definida como "tempo total em teste" e é a estatística natural para inferências relacionadas à distribuição exponencial.

De $l^{\prime}(\theta)=0$, encontramos o estimador de verossimilhança para $\theta$, dado por $\hat{\theta}=T / n$.

Testes de hipóteses e intervalos de confiança para $\theta$ podem ser facilmente obtidos de $2 T / \theta \sim \chi_{(2 n)}^{2}$. Por exemplo, para obtermos um intervalo de confiança para $\theta$ a um nível de confiança $(1-\alpha)$, consideramos, 


$$
P\left[\chi_{(2 n) ; \alpha / 2}^{2} \leq \frac{2 T}{\theta} \leq \chi_{(2 n) ; 1-\alpha / 2}^{2}\right]=(1-\alpha)
$$

onde $\chi_{(2 n) ; p}^{2}$ é o $p$-ésimo quantil da distribuição $\chi_{(2 n)}^{2}$. Portanto, um intervalo de confiança $(1-\alpha)$ para $\theta$ é dado por,

$$
\frac{2 T}{\chi_{(2 n) ; 1-\alpha / 2}^{2}} \leq \theta \leq \frac{2 T}{\chi_{(2 n) ; \alpha / 2}^{2}}
$$

Quando o tamanho da amostra é grande, intervalos de confiança e testes de hipótese para $\theta$ também podem ser aproximados pela distribuição normal assintótica do estimador de máxima verossimilhança $\hat{\theta} \stackrel{a}{\sim} N\left(\theta ; I_{0}^{-1}\right)$,

onde $\quad I_{0}=-\left.\frac{d^{2} l(\theta)}{d \theta^{2}}\right|_{\theta=\hat{\theta}}=\frac{n^{3}}{T^{2}} \quad$ é a informação observada.

\subsubsection{Amostras com Dados Censurados.}

Considere uma amostra aleatória $\left(T_{1}, T_{2}, \ldots, T_{n}\right)$ de tamanho $n$, onde $T_{i}$ representa o tempo de vida do $i$-ésimo componente. Assumindo censuras de tipo II, onde apenas os primeiros $d$ tempos de vida ordenados são observados, ou seja $t_{(1)} \leq t_{(2)} \leq \ldots \leq t_{(d)}$, a função de verossimilhança para $\theta$ é dada por: 


$$
\begin{aligned}
L(\theta) & =\left[\prod_{i=1}^{d} f\left(t_{i}\right)\right]\left[R\left(t_{(d)}\right)\right]^{n-d} \\
& =\frac{1}{\theta^{d}} \exp \left\{-\sum_{i=1}^{d} \frac{t_{(i)}}{\theta}\right\} \exp \left\{-\frac{(n-d) t_{(d)}}{\theta}\right\} \\
& =\frac{1}{\theta^{d}} \mathrm{e}^{-T / \theta},
\end{aligned}
$$

onde $T=\sum_{i=1}^{d} t_{(i)}+(n-d) t_{(d)}$.

Considerando este esquema de censuras, o estimador de máxima verossimilhança é dado por $\hat{\theta}=T / d, d>0$ e $T$ é dado em (3.7).

Testes de hipóteses e intervalos de confiança para $\theta$ podem ser obtidos da distribuição exata $2 T / \theta \sim \chi_{(2 d)}^{2}$ e da aproximação normal para o estimador de máxima verossimilhança $\hat{\theta} \stackrel{a}{\sim} N\left(\theta ; I_{0}^{-1}\right)$, com $I_{0}=d^{3} / T^{2}$. Note que neste caso as expressões são idênticas às do caso anterior, apenas trocando $n$ por $d$. Por este motivo, as amostras completas são consideradas um caso particular de censuras de tipo $\amalg$ onde $d=n$.

Para dados com censuras de tipo $I$, associado a cada indivíduo temos um tempo fixo de censura $L_{i}>0$. Observamos $T_{i}$ somente se $T_{i} \leq L_{i}$, isto é, os dados consistem de pares na forma $\left(t_{i} ; \delta_{i}\right), i=1,2, \ldots, n$ onde:

$$
t_{i}=\min \left(T_{i} ; L_{i}\right) \text { e } \delta_{i}=\left\{\begin{array}{l}
1 \text { se } t_{i}=T_{i} \\
0 \text { se } t_{i}=L_{i}
\end{array}\right.
$$


A função de verossimilhança para $\theta$ com censuras de tipo I é dada por:

$$
\begin{aligned}
L(\theta) & =\prod_{i=1}^{n}\left[f\left(t_{i}\right)\right]^{\delta_{i}}\left[R\left(L_{i}\right)\right]^{1-\delta_{i}} \\
& =\prod_{i=1}^{n}\left[\frac{1}{\theta^{\delta_{i}}} \exp \left\{-\frac{\delta_{i} T_{i}}{\theta}\right\} \exp \left\{-\frac{\left(1-\delta_{i}\right) L_{i}}{\theta}\right\}\right] \\
& =\frac{1}{\theta^{d}} \mathrm{e}^{-T / \theta},
\end{aligned}
$$

onde $d=\sum \delta_{i}$ é o número de tempos de vida realmente observados, e, $T=\sum_{i=1}^{n} t_{i}=\sum_{i \in D} T_{i}+\sum_{i \in C} L_{i}$, onde $D$ e $C$ denotam os conjuntos cujos tempos de vida são observados e censurados, respectivamente.

Como podemos notar, a forma da função de verossimilhança é idêntica ao caso de censuras do tipo II, embora as propriedades amostrais sejam diferentes. Como no caso anterior, o estimador de máxima verossimilhança é dado por $\hat{\theta}=T / d, d>0$. Entretanto, neste caso $d$ é aleatório, e procedimentos para testes de hipótese e intervalos de confiança não são facilmente obtidos, por isso podemos considerar métodos baseados nas propriedades assintóticas do estimador de máxima verossimilhança,

$$
\frac{\hat{\theta}-\theta}{I_{0}^{-1 / 2}} \stackrel{a}{\sim} N(0 ; 1)
$$

onde $I_{0}$ é a informação observada. 


\subsection{Estimação da Confiabilidade de Sistemas com uma Unidade.}

Nesta seção, desenvolveremos técnicas de estimação pontual e por intervalo, para a função de confiabilidade $R\left(t_{0}\right)$ de sistemas com uma unidade, considerando o modelo exponencial para o tempo de vida. Para sistemas deste tipo, a função de confiabilidade num tempo $t_{0}$ é dada por,

$$
R\left(t_{0}\right)=\mathrm{e}^{-t_{0} / \theta}
$$

A função de verossimilhança em termos de $r=R\left(t_{0}\right)$, considerando dados censurados é dada por,

$$
L(r)=\frac{r^{T / t_{0}}}{t_{0}^{d}}(-\ln r)^{d}, \quad 0 \leq r \leq 1
$$

onde $T$ é o tempo total em teste e, o logaritmo da verossimilhança é dado por,

$$
l(r)=-d \ln t_{0}+\frac{T}{t_{0}} \ln r+d \ln (-\ln r) .
$$

Portanto, o estimador de máxima verossimilhança para a confiabilidade num dado tempo $t_{0}$ é dado por:

$$
\hat{R}\left(t_{0}\right)=\mathrm{e}^{-d t_{0} / T}
$$

cuja normalidade assintótica obtida pelo "método delta", baseado na informação observada (ver apêndice C), é dada por: 


$$
\hat{R}\left(t_{0}\right) \stackrel{a}{\sim} N\left[\mathrm{e}^{-t_{0} / \theta} ; \frac{d t_{0}^{2} \mathrm{e}^{-2 d t_{0} / T}}{T^{2}}\right]
$$

que pode ser usada para construir testes de hipóteses e intervalos de confiança para $R\left(t_{0}\right)$. No entanto, em trabalhos práticos, essa aproximação normal pode ser muito pobre comprometendo a precisão das estimativas. Uma alternativa, então, seria obter uma reparametrização que forneça "boa normalidade" para a função de verossimilhança. Portanto, exploramos as reparametrizações de Guerrero e Johnson e Aranda-Ordaz, descritas no capítulo anterior.

\subsubsection{Reparametrização de Guerrero e Johnson para a Confiabilidade.}

Considerando a transformação de Guerrero e Johnson dada em (2.3), a função de verossimilhança para $\phi_{G J}=\phi_{G J}(\lambda)$ é dada por,

$$
\begin{aligned}
L\left(\phi_{G J}\right) & =\frac{1}{t_{0}^{d}}\left\{\ln \left[1+\left(1+\phi_{G J}\right)^{-1 / \lambda}\right]\right\}^{d}\left[1+\left(1+\phi_{G J}\right)^{-1 / \lambda}\right]^{-T / t_{0}} \\
& =\frac{1}{t_{0}^{d}}\left[B\left(\phi_{G J}\right)\right]^{d}\left[1+A\left(\phi_{G J}\right)\right]^{-T / t_{0}}
\end{aligned}
$$

onde $B\left(\phi_{G J}\right)=\ln \left[1+A\left(\phi_{G J}\right)\right]$ e $A\left(\phi_{G J}\right)=\left(1+\phi_{G J}\right)^{-1 / \lambda}$.

O logaritmo da verossimilhança é dado por,

$$
l\left(\phi_{G J}\right)=-d \ln t_{0}+d \ln \left[B\left(\phi_{G J}\right)\right]-\frac{T}{t_{0}} B\left(\phi_{G J}\right)
$$


O estimador de máxima verossimilhança para $\phi_{G J}$, obtido de $l^{\prime}\left(\phi_{G J}\right)=0$, é dado por:

$$
\hat{\phi}_{G J}(\lambda)=\left(\mathrm{e}^{d t_{0} / T}-1\right)^{-\lambda}-1
$$

Para determinar qual é o valor mais apropriado de $\lambda$ que forneça boa normalidade para a função de verossimilhança, utilizamos a terceira derivada padronizada do logaritmo da verossimilhança definida em (2.14). A segunda e terceira derivadas de $l\left(\phi_{G J}\right)$ calculadas localmente no estimador de máxima verossimilhança $\hat{\phi}_{G J}(\lambda)$ (ver apêndice A) são dadas por:

$$
l^{\prime \prime}\left(\hat{\phi}_{G J}\right)=-\frac{T^{2}}{d \lambda^{2} t_{0}^{2}} \mathrm{e}^{-2 d t_{0} / T}\left(\mathrm{e}^{d t_{0} / T}-1\right)^{2(\lambda+1)}
$$

e

$$
l^{\prime \prime \prime}\left(\hat{\phi}_{G J}\right)=\frac{T^{2}\left(\mathrm{e}^{d t_{0} / T}-1\right)^{3(\lambda+1)}}{d \lambda^{2} t_{0}^{2} \mathrm{e}^{2 d t_{0} / T}}\left[\frac{3\left(1+\lambda^{-1} \mathrm{e}^{-d t_{0} / T}\right)}{\mathrm{e}^{d t_{0} / T}-1}-\frac{2 T}{d \lambda t_{0} \mathrm{e}^{d t_{0} / T}}\right]
$$

Portanto, a terceira derivada padronizada $(S T D)$ de $l\left(\phi_{G J}\right)$, localmente em $\hat{\phi}_{G J}(\lambda)$ é dada por:

$$
S T D\left(\hat{\phi}_{G J}\right)=\left|\frac{\lambda t_{0} d^{1 / 2}}{T \mathrm{e}^{-d t_{0} / T}}\left[\frac{3\left(1+\lambda^{-1} \mathrm{e}^{-d t_{0} / T}\right)}{\mathrm{e}^{d t_{0} / T}-1}-\frac{2 T}{d \lambda \mathrm{e}^{d t_{0} / T}}\right]\right|
$$


Assim, o valor de $\lambda$ tal que $S T D\left(\hat{\phi}_{G J}\right)=0$, é dado por:

$$
\lambda=\left(\frac{2 T}{3 d t_{0}}+1\right)\left(1-\mathrm{e}^{-d t_{0} / T}\right)-1
$$

Com $\lambda$ obtido em (3.19) podemos considerar a normalidade assintótica de $\hat{\phi}_{G J}(\lambda)$ dada por,

$$
\hat{\phi}_{G J} \stackrel{a}{\sim} N\left[\phi_{G J} ; \frac{d \lambda^{2} t_{0}^{2} \mathrm{e}^{2 d t_{0} / T}}{T^{2}\left(\mathrm{e}^{d t_{0} / T}-1\right)^{2(\lambda+1)}}\right]
$$

e conseguir melhores inferências para a confiabilidade, especialmente para amostras pequenas e moderadas.

\subsubsection{Reparametrização de Aranda-Ordaz para a Confiabilidade.}

Com a transformação de Aranda-Ordaz dada em (2.5), a função de verossimilhança para $\phi_{A O}=\phi_{A O}(\lambda)$, é dada por,

$$
\begin{aligned}
L\left(\phi_{A O}\right) & =\frac{1}{t_{0}^{d}}\left\{\ln \left[1+\left(\frac{1-\phi_{A O}}{1+\phi_{A O}}\right)^{1 / \lambda}\right]\right\}^{d}\left[1+\left(\frac{1-\phi_{A O}}{1+\phi_{A O}}\right)^{1 / \lambda}\right]^{-T / t_{0}}, \\
& =\frac{1}{t_{0}^{d}}\left[D\left(\phi_{A O}\right)\right]^{d}\left[1+C\left(\phi_{A O}\right)\right]^{-T / t_{0}}
\end{aligned}
$$

onde $D\left(\phi_{A O}\right)=\ln \left[1+C\left(\phi_{A O}\right)\right]$ e $C\left(\phi_{A O}\right)=\left(\frac{1-\phi_{A O}}{1+\phi_{A O}}\right)^{1 / \lambda}$. 
O logaritmo da verossimilhança é dado por:

$$
l\left(\phi_{A O}\right)=-d \ln t_{0}+d \ln \left[D\left(\phi_{A O}\right)\right]-\frac{T}{t_{0}} \ln \left[1+C\left(\phi_{A O}\right)\right]
$$

O estimador de máxima verossimilhança para $\phi_{A O}$, é dado por:

$$
\hat{\phi}_{A O}(\lambda)=\frac{1-\left(\mathrm{e}^{d t_{0} / T}-1\right)^{\lambda}}{1+\left(\mathrm{e}^{d t_{0} / T}-1\right)^{\lambda}}
$$

A segunda e terceira derivadas de $l\left(\phi_{A O}\right)$ calculadas no estimador de máxima verossimilhança $\hat{\phi}_{A O}(\lambda)$ (ver apêndice $B$ ) são dadas por:

$$
l^{\prime \prime}\left(\hat{\phi}_{A O}\right)=-\frac{4 T^{2} \mathrm{e}^{-2 d t_{0} / T}\left(\mathrm{e}^{d t_{0} / T}-1\right)^{2}}{d \lambda^{2} t_{0}^{2}\left(1-\hat{\phi}_{A O}^{2}\right)^{2}}
$$

e

$$
l^{\prime \prime \prime}\left(\hat{\phi}_{A O}\right)=\frac{8 T^{2} \mathrm{e}^{-2 d t_{0} / T}\left(\mathrm{e}^{d t_{0} / T}-1\right)^{2}}{d \lambda^{2} t_{0}^{2}\left(1-\hat{\phi}_{A O}^{2}\right)^{3}}\left[\frac{3 \mathrm{e}^{-d t_{0} / T}}{\lambda}-3 \hat{\phi}_{A O}-\frac{2 T}{d \lambda t_{0}}+\frac{2 T \mathrm{e}^{-d t_{0} / T}}{d \lambda t_{0}}\right] .
$$

Portanto, a terceira derivada padronizada (STD) de $l\left(\phi_{G J}\right)$, localmente em $\hat{\phi}_{G J}(\lambda)$ é dada por:

$$
S T D\left(\hat{\phi}_{A O}\right)=\left|\frac{d^{1 / 2} t_{0} \lambda \mathrm{e}^{d t_{0} / T}}{T\left(\mathrm{e}^{d t_{0} / T}-1\right)}\left[\frac{3 \mathrm{e}^{-d t_{0} / T}}{\lambda}-3 \hat{\phi}_{A O}-\frac{2 T}{d \lambda t_{0}}+\frac{2 T \mathrm{e}^{-d t_{0} / T}}{d \lambda t_{0}}\right]\right|
$$


Para que $\operatorname{STD}\left(\hat{\phi}_{A O}\right)=0$, devemos achar $\lambda$ tal que:

$$
g(\lambda)=\frac{3 \mathrm{e}^{-d t_{0} / T}}{\lambda}+\frac{2 T \mathrm{e}^{-d t_{0} / T}}{d \lambda t_{0}}-\frac{2 T}{d \lambda t_{0}}-3\left[\frac{1-\left(\mathrm{e}^{d t_{0} / T}-1\right)^{\lambda}}{1+\left(\mathrm{e}^{d t_{0} / T}-1\right)^{\lambda}}\right]=0
$$

Como não temos uma forma explícita para resolver (3.27), devemos usar um método iterativo (por exemplo Newton-Raphson). Com $\lambda$ obtido em (3.27), também podemos considerar a normalidade assintótica usual para $\hat{\phi}_{A O}(\lambda)$ baseada na informação observada,

$$
\hat{\phi}_{A O} \stackrel{a}{\sim} N\left(\phi_{A O} ; v^{2}\right)
$$

onde $v^{2}=\frac{4 d \lambda^{2} t_{0}^{2}\left(\mathrm{e}^{d t_{0} / T}-1\right)^{2(\lambda-1)} \mathrm{e}^{2 d t_{0} / T}}{T^{2}\left[1+\left(\mathrm{e}^{d t_{0} / T}-1\right)^{\lambda}\right]^{4}}$

e conseguir melhores inferências para a confiabilidade, especialmente para amostras pequenas e moderadas. 


\section{Capítulo 4}

\section{ESTIMAÇÃO BAYESIANA PARA A CONFIABILIDADE DE SISTEMAS COM UMA UNIDADE CONSIDERANDO A DISTRIBUIÇÃO EXPONENCIAL.}

Em análise da confiabilidade, quando assumimos um modelo para o tempo de vida dos componentes, temos interesse em determinar o valor do parâmetro que o define. Embora este parâmetro seja considerado fixo, não temos certeza do seu verdadeiro valor, e assim procuramos uma forma de, através dos dados, expressar essa nossa incerteza .

Para o caso uniparamétrico, assumindo que os tempos de vida $T_{1} ; T_{2} ; \ldots ; T_{n}$ sejam independentes, a função de verossimilhança $L(\theta)$ nos fornece um meio de quantificar a informação contida nos dados acerca do verdadeiro valor do parâmetro desconhecido $\theta$. Uma medida que melhor sintetiza esta informação é o estimador de máxima verossimilhança (e.m.v.) $\hat{\theta}$, que é o valor que maximiza $L(\theta)$. O e.m.v. é um estimador muito satisfatório para tamanhos de amostras suficientemente grandes, porém, para modelos muito complexos, o e.m.v. pode ser completamente equivocado, mesmo nas condições de amostras grandes (ver por exemplo Basu, 1975).

Uma boa maneira de expressar nossa incerteza é através de uma distribuição de probabilidade para o parâmetro $\theta$, que sob este ponto de vista é considerado uma variável aleatória. Com esta formulação, usamos o teorema de Bayes (ver por exemplo, 
Box e Tiao, 1973) que fornece um método para computar a densidade de probabilidade de $\theta$, expressando a nossa incerteza condicionada aos dados observados.

\subsection{Fórmula de Bayes.}

Sejam $X$ e $\theta$ variáveis aleatórias com densidades conjunta e condicionais dadas por $p(x, \theta), p(x / \theta)$ e $p(\theta / x)$, respectivamente e seja $p(\theta)$ a densidade marginal de $\theta$. A densidade condicional de $\theta$ dado $x$ é dada por:

$$
p(\theta / x)=\frac{p(x / \theta) p(\theta)}{\int_{\theta \in \Theta} p(x / \theta) p(\theta) d \theta}
$$

onde $\Theta$ é o espaço paramétrico.

\subsection{Densidades a Priori e a Posteriori.}

Antes de analisar estatisticamente os dados é útil acessar nossos conhecimentos a respeito dos parâmetros do modelo. Uma forma conveniente de se fazer isto é formular uma densidade de probabilidade para seu parâmetro $\theta$. Essa densidade de probabilidade para $\theta$ é identificada como densidade a priori, e é denotada por $p_{0}(\theta)$. Quando não temos nenhuma informação a respeito de $\theta$, devemos formular uma densidade a priori tal que toda informação acerca do parâmetro venha exclusivamente dos dados, ou seja, devemos considerar uma densidade a priori não-informativa. Existem várias regras para se formular uma densidade a priori não-informativa, entre as quais destacamos as regras de Jeffreys, Zellner e Bernardo. Uma vez definidos o modelo para os dados e a distribuição a priori, devemos combinar a informação prévia sobre o parâmetro $\theta$ com a informação contida nos dados, obtendo assim uma distribuição a posteriori para $\theta$. 
Supondo uma amostra aleatória com $n$ tempos de vida $t_{1} ; t_{2} ; \ldots ; t_{n}$ com densidade conjunta $p\left(t_{1} ; t_{2} ; \ldots ; t_{n} / \theta\right)$, pela fórmula de Bayes dada em (4.1), a densidade a posteriori para $\theta$ é dada por:

$$
p(\theta / \text { dados })=\frac{p\left(t_{1} ; t_{2} ; \ldots ; t_{n} / \theta\right) p_{0}(\theta)}{\int_{\theta \in \Theta} p\left(t_{1} ; t_{2} ; \ldots ; t_{n} / \theta\right) p_{0}(\theta) d \theta}
$$

Observando que a verossimilhança para $\theta$ é tal que $L(\theta)=p\left(t_{1} ; t_{2} ; \ldots ; t_{n} / \theta\right)$, podemos escrever

$$
p(\theta / \text { dados })=\frac{L(\theta) p_{0}(\theta)}{\int_{\theta \in \Theta} L(\theta) p_{0}(\theta) d \theta}
$$

uma vez que $t_{1} ; t_{2} ; \ldots ; t_{n}$ foram observados, não são mais considerados variáveis neste estágio da análise.

Estimadores Bayesianos devem ser obtidos da densidade a posteriori para $\theta$, pois esta combina a informação prévia sobre o parâmetro com as informações da amostra.

A seguir, apresentaremos os resultados da análise Bayesiana considerando a densidade a priori não-informativa de Jeffreys e a densidade a priori $\operatorname{Beta}\left(\alpha_{0}, \beta_{0}\right)$, para as parametrizações introduzidas nas seções anteriores. 


\subsection{Análise Bayesiana para a Confiabilidade de Sistemas Considerando uma Densidade a Priori Não-Informativa de Jeffreys e Dados Censurados.}

Pela regra de Jeffreys uma densidade a priori não-informativa para $\theta$ no caso uniparamétrico, é dada por,

$$
p_{0}(\theta) \propto[I(\theta)]^{1 / 2}
$$

onde $I(\theta)=E\left[-\frac{d^{2} l(\theta)}{d \theta^{2}}\right]$ é a informação de Fisher, e $\propto$ indica proporcionalidade (ver por exemplo, Box e Tiao, 1973).

Para o caso multiparamétrico, a regra de Jeffreys é dada por,

$$
p_{0}(\theta) \propto[\operatorname{det} \mathbf{I}(\theta)]^{1 / 2}
$$

onde $\mathbf{I}(\theta)$ é a matriz de informação de Fisher.

Considerando o modelo exponencial, definido no capítulo anterior em (3.1), a segunda derivada do logaritmo da verossimilhança dado em (3.9), é dado por:

$$
\frac{d^{2} l(r)}{d r^{2}}=-\frac{d}{(-\ln r)^{2} r^{2}}+\frac{d}{(-\ln r)^{2} r}-\frac{T}{r^{2} t_{0}}
$$

E a informação de Fisher é então: 


$$
I(r)=E\left[-\frac{d^{2} l(r)}{d r^{2}}\right]=\frac{d}{(-\ln r)^{2} r^{2}}-\frac{d}{(-\ln r)^{2} r}+\frac{E(T)}{r^{2} t_{0}}
$$

Como $E(T)=d \theta$, ver Miller 1981, e como $\theta=t_{0}(-\ln r)^{-1}$, a informação de Fisher é dada por:

$$
I(r)=\frac{d}{(-\ln r)^{2} r^{2}}, \quad 0 \leq r \leq 1
$$

Portanto, uma densidade a priori não-informativa para $r=R\left(t_{0}\right)$, segundo a regra de Jeffreys é dada por:

$$
p_{0}(r) \propto \frac{1}{(-\ln r) r}, \quad 0 \leq r \leq 1 .
$$

Considerando a densidade a priori (4.8), a densidade a posteriori para $r$, obtida de (4.2) é dada por,

$$
\begin{aligned}
p_{1}(r / \text { dados })= & \frac{t_{0}^{-d}(-\ln r)^{d} r^{T / t_{0}}[(-\ln r) r]^{-1}}{\int_{0}^{1} t_{0}^{-d}(-\ln r)^{d} r^{T / t_{0}}[(-\ln r) r]^{-1} d r} \\
& =\frac{\left(T / t_{0}\right)^{d}}{\Gamma(d)}(-\ln r)^{d-1} r^{T / t_{0}-1}, \quad 0 \leq r \leq 1,
\end{aligned}
$$

onde $T=\sum_{i=1}^{d} t_{(i)}+(n-d) t_{(d)}$ (uma densidade log-gama negativa). 
Um estimador Bayesiano para o parâmetro de interesse é dado pela moda $\tilde{R}\left(t_{0}\right)$ da densidade a posteriori. Portanto, com a densidade a priori não-informativa de Jeffreys, a moda de (4.9) dada por $\tilde{R}_{1}\left(t_{0}\right)=\mathrm{e}^{-(d-1) t_{0} /\left(T-t_{0}\right)}$, é um estimador Bayesiano para a confiabilidade num dado tempo $t_{0}$.

Outra forma de se obter estimativas Bayesianas é considerar funções de perda para o parâmetro de interesse. Uma função de perda muito comum é a função de perda quadrática dada por,

$$
G(r)=(r-\hat{r})^{2}
$$

onde $\hat{r}$ é o estimador que minimiza a perda esperada com relação à densidade a posteriori.

Considerando a função de perda quadrática, podemos mostrar facilmente que o estimador Bayesiano para o parâmetro de interesse é dado pelo valor esperado da densidade a posteriori. Desta forma, considerando o modelo exponencial para os tempos de vida, a esperança a posteriori de (4.9) dada por

$$
E(R / \text { dados })=\left(1+\frac{t_{0}}{T}\right)^{-d}
$$

é um estimador Bayesiano para a confiabilidade do sistema num dado tempo $t_{0}$.

Considerando a reparametrização de Guerrero e Johnson dada em (2.3) e com $\lambda$ dado em (3.19), obtido de $S T D(\hat{\theta})=0$, o Jacobiano da transformação é dado por:

$$
J\left(r, \phi_{G J}\right)=\frac{d r}{d \phi_{G J}}=\frac{\left(1-\phi_{G J}\right)^{-(1+1 / \lambda)}}{\lambda}\left[1+A\left(\phi_{G J}\right)\right]^{-2} .
$$


Desta forma, a densidade a posteriori para $\phi_{G J}(\lambda)$, assumindo uma densidade a priori não-informativa de Jeffreys, é dada por,

$$
\begin{aligned}
& \begin{aligned}
p_{1}\left(\phi_{G J} / \text { dados }\right)= & \frac{\left(1-\phi_{G J}\right)^{-(1+1 / \lambda)}}{\lambda}\left[1+A\left(\phi_{G J}\right)\right]^{-2} \frac{\left(T / t_{0}\right)^{d}}{\Gamma(d)}\left[B\left(\phi_{G J}\right)\right]^{d-1}\left[1+A\left(\phi_{G J}\right)\right]^{-\left(T / t_{0}-1\right)}, \\
& =\frac{\left(T / t_{0}\right)^{d}}{\lambda \Gamma(d)}\left[B\left(\phi_{G J}\right)\right]^{d-1}\left[1+A\left(\phi_{G J}\right)\right]^{-\left(T / t_{0}+1\right)}\left(\phi_{G J}+1\right)^{-(1 / \lambda+1)}
\end{aligned} \\
& \text { onde }-1 \leq \phi_{G J}<\infty, B\left(\phi_{G J}\right)=\ln \left[1+A\left(\phi_{G J}\right)\right] \text { e } A\left(\phi_{G J}\right)=\left(1+\phi_{G J}\right)^{-1 / \lambda} .
\end{aligned}
$$

Como a moda e o valor esperado de (4.13) não são possíveis de se obter analiticamente, devemos determiná-los através de métodos numéricos ou de aproximação de integrais, como por exemplo o método de Laplace (ver apêndice D).

Da mesma forma, considerando a parametrização de Aranda-Ordaz (2.5), com $\lambda$ obtido de (3.27), a densidade a posteriori de $\phi_{A O}(\lambda)$, assumindo a densidade a priori (4.8), é dada por:

$$
\begin{aligned}
p_{1}\left(\phi_{A O} / \text { dados }\right)= & \frac{2\left(T / t_{0}\right)^{d}}{\lambda \Gamma(d)}\left[D\left(\phi_{A O}\right)\right]^{d-1}\left[1+C\left(\phi_{A O}\right)\right]^{-\left(T / t_{0}+1\right)} \\
& \left(\frac{1-\phi_{A O}}{1+\phi_{A O}}\right)^{2 / \lambda} \frac{1}{\left(1-\phi_{A O}^{2}\right)}
\end{aligned}
$$

onde $-1 \leq \phi_{A O} \leq 1, D\left(\phi_{A O}\right)=\ln \left[1+C\left(\phi_{A O}\right)\right]$ e $C\left(\phi_{A O}\right)=\left(\frac{1-\phi_{A O}}{1+\phi_{A O}}\right)^{1 / \lambda}$. 
Novamente devemos recorrer a métodos numéricos ou de aproximação de integrais para a obtenção da esperança e da moda de (4.14).

\subsection{Análise Bayesiana para a Confiabilidade de Sistemas Considerando uma Densidade a Priori Beta e Dados Censurados.}

$\mathrm{Na}$ parametrização original, uma densidade a priori informativa para $R\left(t_{0}\right)$ considerada na literatura (ver por exemplo Martz e Waller, 1982) é dada pela densidade $\operatorname{Beta}\left(\alpha_{0}, \beta_{0}\right)$,

$$
g\left(r ; \alpha_{0}, \beta_{0}\right)=\frac{\Gamma\left(\alpha_{0}+\beta_{0}\right)}{\Gamma\left(\alpha_{0}\right) \Gamma\left(\beta_{0}\right)} r^{\alpha_{0}-1}(1-r)^{\beta_{0}-1}, \quad 0 \leq r \leq 1 ; \alpha_{0}, \beta_{0}>0
$$

A densidade a posteriori para a confiabilidade de sistemas num dado tempo $t_{0}$, considerando a densidade a priori (4.15) é dada por,

$$
p_{2}(r / \text { dados })=\frac{(-\ln r)^{d} r^{\alpha_{0}+T / t_{0}-1}(1-r)^{\beta_{0}-1}}{\int_{0}^{1}(-\ln x)^{d} x^{\alpha_{0}+T / t_{0}-1}(1-x)^{\beta_{0}-1} d x}, \quad 0 \leq r \leq 1 .
$$

Assumindo $\alpha_{0}=\beta_{0}=1$, em (4.15) temos uma densidade a priori uniforme para $R\left(t_{0}\right)$. A densidade a posteriori em (4.16) com $\alpha_{0}=\beta_{0}=1$ é dada por,

$$
p_{2}(r / \text { dados })=\frac{\left(1+T / t_{0}\right)^{d+1}}{\Gamma(d+1)}(-\ln r)^{d} r^{T / t_{0}}, \quad 0 \leq r \leq 1
$$


A moda de (4.17) é dada por $\tilde{R}\left(t_{0}\right)=\mathrm{e}^{-d t_{0} / T}$, que coincide com o estimador de máxima verossimilhança e, a esperança a posteriori de (4.17) é dada por,

$$
E(R / \text { dados })=\left(\frac{1+T / t_{0}}{2+T / t_{0}}\right)^{d+1}
$$

Nas parametrizações de Guerrero e Johnson e Aranda-Ordaz, as densidades a posteriori para $\phi_{G J}(\lambda)$ e $\phi_{A O}(\lambda)$ considerando a densidade a priori $\operatorname{Beta}\left(\alpha_{0}, \beta_{0}\right)$, com $\alpha_{0}=\beta_{0}=1$, são dadas, respectivamente, por:

$$
p_{2}\left(\phi_{G J} / \text { dados }\right)=\frac{\left(1+T / t_{0}\right)^{d+1}}{\lambda \Gamma(d+1)}\left[B\left(\phi_{G J}\right)\right]^{d}\left[1+A\left(\phi_{G J}\right)\right]^{-\left(T / t_{0}+2\right)}\left(\phi_{G J}+1\right)^{-(1 / \lambda+1)},
$$

onde $-1 \leq \phi_{G J}<\infty$ e $A\left(\phi_{G J}\right)$ e $B\left(\phi_{G J}\right)$ são dados em (4.13), e,

$$
\begin{aligned}
p_{2}\left(\phi_{A O} / \text { dados }\right)= & \frac{2\left(1+T / t_{0}\right)^{d+1}}{\lambda \Gamma(d+1)}\left[D\left(\phi_{A O}\right)\right]^{d}\left[1+C\left(\phi_{A O}\right)\right]^{-\left(T / t_{0}+2\right)} \\
& \left(\frac{1-\phi_{A O}}{1+\phi_{A O}}\right)^{4 / \lambda} \frac{1}{\left(1-\phi_{A O}^{2}\right)}
\end{aligned}
$$

onde $-1 \leq \phi_{A O} \leq 1$, e $C\left(\phi_{A O}\right)$ e $D\left(\phi_{A O}\right)$ são dados em (4.14).

Para se obter o moda e esperança a de (4.19) e (4.20) devemos utilizar métodos numéricos ou de aproximação de integrais, pois não temos uma solução analítica. 


\section{Capítulo 5}

\section{REPARAMETRIZAÇÃo PARA A CONFIABILIDADE CONSIDERANDO O MODELO DE WEIBULL E DADOS CENSURADOS.}

Neste capítulo estendemos as técnicas de reparametrização para a distribuição de Weibull. Uma variável aleatória $T$ tem distribuição de Weibull se a sua f.d.p. for da forma:

$$
f(t ; \alpha, \beta)=\frac{\beta}{\alpha}\left(\frac{t}{\alpha}\right)^{\beta-1} \exp \left\{-\left(\frac{t}{\alpha}\right)^{\beta}\right\}, \quad t \geq 0
$$

onde $\alpha, \beta>0$ são parâmetros de forma e escala, respectivamente.

A distribuição de Weibull é uma distribuição particularmente importante nos estudos de tempos de vida, e muitos métodos estatísticos tem sido desenvolvidos para ela. Uma razão para que tantos artigos sobre a distribuição de Weibull sejam escritos, diz respeito às suas propriedades estatísticas. Em geral não há estatística suficiente bidimensional para os parâmetros $\alpha$ e $\beta$, e as possibilidades de se produzir estimadores são muitas. Soma-se a isso o fato de que as distribuições de muitos estimadores e outras estatísticas associadas à distribuição de Weibull serem matematicamente 
intratáveis. Atualmente dispomos de procedimentos estatísticos que têm se mostrado relativamente fáceis graças ao desenvolvimento dos computadores.

\subsection{Inferências para a Distribuição de Weibull.}

Seja uma amostra aleatória de tamanho $n$, com tempos de vida $T_{1}, T_{2}, \ldots, T_{n}$, distribuídos segundo a distribuição de Weibull dada em (5.1). A função de confiabilidade num dado tempo $t_{0}$, é dada por:

$$
R\left(t_{0}\right)=\exp \left[-\left(t_{0} / \alpha\right)^{\beta}\right], \quad t_{0} \geq 0
$$

Observe que, para $\beta=1$ temos o modelo exponencial dado em (3.1).

\subsubsection{Amostras Completas.}

A função de verossimilhança para os parâmetros $\alpha$ e $\beta$, considerando amostras sem censuras, é dada por:

$$
L(\alpha, \beta)=\left(\frac{\beta}{\alpha^{\beta}}\right)^{n}\left[\prod_{i=1}^{n}\left(t_{i}\right)^{\beta-1}\right] \exp \left\{-\sum_{i=1}^{n}\left(\frac{t_{i}}{\alpha}\right)^{\beta}\right\} .
$$

e o logaritmo da função de verossimilhança é dado por:

$$
l(\alpha, \beta)=n \ln \beta-n \beta \ln \alpha+(\beta-1) \sum_{i=1}^{n} \ln t_{i}-\sum_{i=1}^{n}\left(\frac{t_{i}}{\alpha}\right)^{\beta} .
$$


Os estimadores de máxima verossimilhança dos parâmetros $\alpha$ e $\beta$ são obtidos através das derivadas parciais:

$$
\left\{\begin{array}{l}
\frac{\partial l(\alpha, \beta)}{\partial \alpha}=-\frac{n \beta}{\alpha}+\frac{\beta}{\alpha} \sum_{i=1}^{n}\left(\frac{t_{i}}{\alpha}\right)^{\beta} \\
\frac{\partial l(\alpha, \beta)}{\partial \beta}=\frac{n}{\beta}-d \ln \alpha+\sum_{i=1}^{n} \ln t_{i}-\sum_{i=1}^{n}\left(\frac{t_{i}}{\alpha}\right)^{\beta} \ln \left(\frac{t_{i}}{\alpha}\right)
\end{array}\right.
$$

Tomando $\partial l / \partial \alpha=0$ e $\partial l / \partial \beta=0, \hat{\alpha}$ é dado pela relação:

$$
\hat{\alpha}=\left[\sum_{i=1}^{n} \frac{\left(t_{i}\right)^{\hat{\beta}}}{n}\right]^{1 / \hat{\beta}},
$$

porém, $\hat{\beta}$ não tem uma forma explícita, sendo necessário a utilização de um método iterativo para resolver:

$$
\frac{n}{\hat{\beta}}-n \frac{\sum_{i=1}^{n} t_{i}^{\hat{\beta}} \ln t_{i}}{\sum_{i=1}^{n} t_{i}^{\hat{\beta}}}+\sum_{i=1}^{n} \ln t_{i}=0 .
$$

Intervalos de confiança e testes de hipótese para os parâmetros $\alpha$ e $\beta$ são usualmente aproximados pela distribuição normal assintótica dos estimadores de máxima verossimilhança:

$$
(\hat{\alpha}, \hat{\beta}) \stackrel{a}{\sim} N\left[(\alpha, \beta) ; \mathbf{I}_{0}^{-1}\right]
$$

onde $\mathbf{I}_{\mathbf{0}}$ é a matriz de informação observada. 


\subsubsection{Amostras Censuradas.}

Assumindo censuras de tipo II, a função de verossimilhança para os parâmetros $\alpha$ e $\beta$ é dada por:

$$
\begin{aligned}
L(\alpha, \beta) & =\left[\prod_{i=1}^{d} f\left(t_{(i)}\right)\right]\left[R\left(t_{(d)}\right)\right]^{n-d} \\
& =\left(\frac{\beta}{\alpha^{\beta}}\right)^{d} \prod_{i \in D} t_{(i)}^{\beta-1} \exp \left\{-\sum_{i=1}^{d}\left(\frac{t_{(i)}}{\alpha}\right)^{\beta}-(n-d)\left(\frac{t_{(d)}}{\alpha}\right)^{\beta}\right\} \\
& =\left(\frac{\beta}{\alpha^{\beta}}\right)^{d} \prod_{i \in D} t_{(i)}^{\beta-1} \exp \left\{-\frac{T(\beta)}{\alpha^{\beta}}\right\},
\end{aligned}
$$

onde: $T(\beta)=\sum_{i=1}^{d} t_{(i)}^{\beta}-(n-d) t_{(d)}^{\beta}$, $d$ é o número de falhas observadas e $D$ é o conjunto de dados não censurados.

O logaritmo da função de verossimilhança para $\alpha$ e $\beta$ é dado por:

$$
l(\alpha, \beta)=d \ln \beta-d \beta \ln \alpha+(\beta-1) \sum_{i \in D} \ln t_{(i)}-\frac{T(\beta)}{\alpha^{\beta}}
$$

Os estimadores de máxima verossimilhança são obtidos de $\partial l / \partial \alpha=0$ e $\partial l / \partial \beta=0$, da mesma forma como no caso anterior.

Testes de hipótese e intervalos de confiança também são obtidos através da normalidade assintótica dos estimadores de máxima verossimilhança. 
No caso de censuras de tipo $I$, a função de verossimilhança é dada por:

$$
\begin{aligned}
L(\alpha, \beta) & =\prod_{i=1}^{n}\left[f\left(t_{i}\right)\right]^{\delta_{i}}\left[R\left(L_{i}\right)\right]^{1-\delta_{i}} \\
& =\left(\frac{\beta}{\alpha^{\beta}}\right)^{d} \prod_{i \in D} t_{i}^{\beta-1} \exp \left\{-\frac{T(\beta)}{\alpha^{\beta}}\right\},
\end{aligned}
$$

onde: $T(\beta)=\sum_{i \in D} T_{i}^{\beta}+\sum_{i \in C} L_{i}^{\beta}, d=\sum_{i=1}^{n} \delta_{i}$ é o número de falhas observadas e $D$ e $C$ são os conjuntos cujos tempos de vida são observados e censurados, respectivamente.

\subsection{Estimação da confiabilidade de Sistemas com uma Unidade.}

As técnicas de estimação pontual da função de confiabilidade $R\left(t_{0}\right)$, são basicamente as mesmas que no caso exponencial, apenas com o agravante de que agora temos um modelo com dois parâmetros.

A função de confiabilidade num tempo $t_{0}$ para sistemas com uma unidade, segundo o modelo de Weibull, é dada por:

$$
R\left(t_{0}\right)=\exp \left\{-\left(\frac{t_{0}}{\alpha}\right)^{\beta}\right\}
$$

De (5.12) temos que $\alpha=t_{0}(-\ln r)^{-1 / \beta}$, onde $r=R\left(t_{0}\right)$. Portanto, com censuras de tipo II, o logaritmo da função verossimilhança para $\beta$ e $r$ é dado por: 
Utilizando o método iterativo de Newton-Raphson encontramos o valor de $\hat{\beta}$ que maximiza (5.7) e, conseqüentemente, obtemos $\hat{r}$ por:

$$
\hat{r}=\exp \left\{-\frac{d t_{0}^{\hat{\beta}}}{T(\hat{\beta})}\right\}
$$

O vetor de estimadores de máxima verossimilhança $(\hat{r}, \hat{\beta})$, considerando amostras grandes, tem distribuição assintótica normal bivariada dada por, $(\hat{r}, \hat{\beta}) \stackrel{a}{\sim} N_{2}\left[(r, \beta) ; \mathbf{I}_{0}^{-1}\right]$, onde $\mathbf{I}_{0}$ é a matriz de informação observada.

Na prática, podemos ter dificuldades nas inferências sobre $r$ e $\beta$ devido ao fato da existência de dois parâmetros no modelo de Weibull; desta forma, o conhecimento prévio do parâmetro $\beta$ torna a inferência sobre $r$ muito mais simples. Portanto, trataremos da estimação da função de confiabilidade sob os dois enfoques: $\beta$ conhecido e $\beta$ desconhecido.

\subsubsection{Inferências para o Modelo de Weibull $\operatorname{com} \boldsymbol{\beta}$ Conhecido.}

Quando o valor de $\beta$ é conhecido, o problema se reduz à estimação de um único parâmetro $r$, simplificando bastante as análises. Nesta situação caímos num caso especial da distribuição exponencial, pois se uma variável aleatória $Y$ tem distribuição de Weibull com parâmetros $\alpha$ e $\beta$, ou seja $Y \sim W e i(\alpha, \beta)$, então a variável aleatória definida por $Y^{\beta}$ tem distribuição exponencial $Y^{\beta} \sim \operatorname{Exp}\left(\alpha^{\beta}\right)$. Portanto, o logaritmo da função de verossimilhança pode ser escrito por: 


$$
l(r) \propto d \ln (-\ln r)+\frac{T}{t_{0}^{\beta}} \ln r
$$

onde: $T=\sum_{i=1}^{d} t_{(i)}^{\beta}+(n-d) t_{(d)}^{\beta}$.

As inferências, então, serão as mesmas que para o modelo exponencial, e o estimador de máxima verossimilhança para $R\left(t_{0}\right)$, obtido de $l^{\prime}(r)=0$, é dado por $\hat{r}=\mathrm{e}^{-d t_{0}^{\beta} / T}$, tendo distribuição normal assintótica dada por:

$$
\hat{r} \stackrel{a}{\sim} N\left[r ; \mathbf{I}_{0}^{-1}\right], \text { onde } \mathbf{I}_{0} \text { é a informação observada. }
$$

A segunda e terceira derivadas de $l(r)$, localmente em $\hat{r}$ são dadas por,

$$
\left\{\begin{array}{l}
l^{\prime \prime}(\hat{r})=-\frac{d}{\hat{r}^{2}(\ln \hat{r})^{2}} \\
l^{\prime \prime \prime}(\hat{r})=\frac{3 d}{\hat{r}^{3}(\ln \hat{r})^{2}}+\frac{2 d}{\hat{r}^{3}(\ln \hat{r})^{3}}
\end{array}\right.
$$

e a terceira derivada padronizada do logaritmo da função de verossimilhança (ver Kass \& Slate 1992), é dada por:

$$
\operatorname{STD}(\hat{r})=\left|d^{-1 / 2}(3 \ln \hat{r}+2)\right|
$$

Para diagnóstico da normalidade da função de verossimilhança, o T-plot de Hills e Smith (1993), é dado por 
$T(\hat{r})=\operatorname{sgn}(r-\hat{r})\left\{-2\left[d \ln \beta-d \beta \ln t_{0}+(\beta-1) \sum_{i \in D} \ln t_{(i)}+d \ln (-\ln r)+(\ln r) \frac{T}{t_{0}^{\beta}}-l(\hat{r})\right]\right\}^{1 / 2}$

\subsubsection{Reparametrização de Guerrero e Johnson para a Confiabilidade $\operatorname{com} \beta$ Conhecido.}

Visando melhorar a normalidade da função de verossimilhança para $R\left(t_{0}\right)$, seja a transformação de Guerrero e Johnson, com $r=R\left(t_{0}\right)$, dada por:

$$
\phi_{G J}(\lambda)=\left(\frac{r}{1-r}\right)^{\lambda}-1 \text { e } \theta=\ln \beta \text { (conhecido) }
$$

O logaritmo da função de verossimilhança para $\phi_{G J}$, com $\phi_{G J}=\phi_{G J}(\lambda)$, é dado por:

$$
l\left(\phi_{G J}\right) \propto d \ln \left\{\ln \left[1+\left(1+\phi_{G J}\right)^{-1 / \lambda}\right]\right\}-\ln \left[1+\left(1+\phi_{G J}\right)^{-1 / \lambda}\right] \frac{T}{t_{0}^{\mathrm{e}^{\theta}}}
$$

Definindo $\quad A\left(\phi_{G J}\right)=\left(1+\phi_{G J}\right)^{-1 / \lambda} \quad$ e $\quad B\left(\phi_{G J}\right)=\ln \left[1+A\left(\phi_{G J}\right)\right], \quad$ com $T=\sum_{i=1}^{d} t_{(i)}^{e^{\theta}}+(n-d) t_{(d)}^{e^{\theta}},(5.23)$ pode ser reescrito como:

$$
l\left(\phi_{G J}\right) \propto d \ln \left[B\left(\phi_{G J}\right)\right]-B\left(\phi_{G J}\right) \frac{T}{t_{0}^{e^{\theta}}}
$$


e o estimador de máxima verossimilhança $\hat{\phi}_{G J}(\lambda)$, obtido de $l^{\prime}\left(\phi_{G J}\right)=0$, é dado por:

$$
\hat{\phi}_{G J}(\lambda)=\left[\mathrm{e}^{d t_{0}^{8} / T}-1\right]^{-\lambda}-1
$$

A terceira derivada padronizada de $\operatorname{STD}\left(\phi_{G J}\right)$, localmente em $\hat{\phi}_{G J}(\lambda)$, é dada por:

$$
\operatorname{STD}\left(\hat{\phi}_{G J}\right)=\left|d^{-1 / 2}\left\{2-\frac{3 B\left(\hat{\phi}_{G J}\right) B^{\prime \prime}\left(\hat{\phi}_{G J}\right)}{\left[B^{\prime}\left(\hat{\phi}_{G J}\right)\right]^{2}}\right\}\right|
$$

Portanto, de (5.26), encontramos um valor apropriado de $\lambda$, tal que $\operatorname{STD}\left(\hat{\phi}_{G J}\right)=0$, dado por:

$$
\lambda=\left(\frac{2 T}{3 d t_{0}^{\mathrm{e}^{\mathrm{e}}}}+1\right)\left(1-\mathrm{e}^{-d t_{0}^{e^{\theta}} / \mathrm{T}}\right)-1
$$

Observe que, $\operatorname{com} \beta=1(\theta=0)$, temos o caso exponencial e a expressão (5.27) se reduz a (3.19).

\subsubsection{Inferências para o Modelo de Weibull $\operatorname{com} \beta$ Desconhecido.}

No caso em que $\beta$ é desconhecido, não é simples se obter uma transformação conjunta que forneça normalidade para $R\left(t_{0}\right)$ e $\beta$ ao mesmo tempo (ver por exemplo Sprott, 1980 ou Kass e Slate, 1992). Uma alternativa, portanto, seria obter uma 
transformação para $R\left(t_{0}\right)$ que forneça boa normalidade para a verossimilhança "profile" $L_{\hat{\beta}}(r)=L(r, \hat{\beta})$, onde $\hat{\beta}$ é o valor que maximiza o logaritmo da verossimilhança para cada valor de $R\left(t_{0}\right)$. Portanto o logaritmo da verossimilhança "profile", com $r=R\left(t_{0}\right)$ é dado por:

$$
l_{\hat{\beta}}(r)=d \ln \hat{\beta}-d \hat{\beta} \ln t_{0}+(\hat{\beta}-1) \sum_{i \in D} \ln t_{(i)}+d \ln (-\ln r)+(\ln r) \frac{T(\hat{\beta})}{t_{0}^{\hat{\beta}}}
$$

onde: $T(\hat{\beta})=\sum_{i=1}^{d} t_{(i)}^{\hat{\beta}}+(n-d) t_{(d)}^{\hat{\beta}}$ e $\hat{\beta}$ é o estimador que maximiza o logaritmo da verossimilhança dado em (5.13), para cada valor de $r$.

O T-plot de Hills e Smith deve ser obtido ponto a ponto pela relação:

$$
T(r)=\operatorname{sgn}(r-\hat{r})\left\{-2\left[l_{\hat{\beta}}(r)-l(\hat{r} ; \hat{\beta})\right]\right\} .
$$

onde $\hat{\beta}$ maximiza (5.13) para cada valor de $r$.

Considerando a transformação de Guerrero e Johnson, $\phi_{G J}(\lambda)=[r /(1-r)]^{\lambda}-1$, onde $r=R\left(t_{0}\right)$ e $\theta=\ln \beta$, o logaritmo da função de verossimilhança profile de $\phi_{G J}(\lambda)$ é dada por:

$$
l_{\hat{\theta}}\left(\phi_{G J}\right)=d \hat{\theta}-d \mathrm{e}^{\hat{\theta}} \ln t_{0}+d \ln \left[B\left(\phi_{G J}\right)\right]+\left(\mathrm{e}^{\hat{\theta}}-1\right) \sum_{i \in D} \ln t_{(i)}-B\left(\phi_{G J}\right) \frac{T(\hat{\theta})}{t_{0}^{\mathrm{e}^{\hat{\theta}}}}
$$

onde $T(\hat{\theta})=\sum_{i=1}^{d} t_{(i)}^{\mathrm{e}^{\hat{\mathrm{\theta}}}}+(n-d) t_{(d)}^{\mathrm{e}^{\hat{\mathrm{\theta}}}}$ e $\hat{\theta}$ é o estimador que maximiza o logaritmo da função de verossimilhança para cada valor de $\phi_{G J}(\lambda)$. 
Para encontrar um valor apropriado de $\lambda$ podemos considerar a terceira derivada padronizada de $l_{\hat{\theta}}\left(\phi_{G J}\right)$, calculada localmente em $\hat{\phi}_{G J}(\lambda)$, dada por:

$$
S T D\left(\hat{\phi}_{G J}\right)=\left|d^{-1 / 2}\left[2-\frac{3 d t_{0}^{e^{\hat{\theta}}}\left\{(1+\lambda)\left[1+\left(1+\hat{\phi}_{G J}\right)^{1 / \lambda}\right]-1\right\}}{T(\hat{\theta})}\right]\right|
$$

onde $\hat{\phi}_{G J}(\lambda)=\left[\mathrm{e}^{d t_{0}^{t_{0}^{\hat{\theta}}} / T(\hat{\theta})}-1\right]^{-\lambda}-1$ e $\hat{\theta}$ é tal que:

$$
\sum_{i \in D} \ln t_{i}+\frac{d}{\mathrm{e}^{\hat{\theta}}}-d \frac{U(\hat{\theta})}{T(\hat{\theta})}=0
$$

$\operatorname{com} T(\hat{\theta})=\sum_{i=1}^{d} t_{(i)}^{\hat{e}^{\hat{\theta}}}+(n-d) t_{(d)}^{\mathrm{e}^{\hat{\theta}}}$ e $U(\hat{\theta})=\sum_{i=1}^{d} t_{(i)}^{\hat{e}^{\hat{\theta}}} \ln t_{(i)}+(n-d) t_{(d)}^{\mathrm{e}^{\hat{\theta}}} \ln t_{(d)}$

Na prática, devemos encontrar um valor de $\lambda$ tal que $\operatorname{STD}\left(\hat{\phi}_{G J}\right) \approx 0$, considerando um gráfico de $\operatorname{STD}\left(\hat{\phi}_{G J}\right)$ para diferentes valores de $\lambda$.

Considerando, ainda, a verossimilhança conjunta para $r$ e $\beta$, podemos encontrar $\lambda$ através da medida de não normalidade de Kass e Slate, definida em (2.13), dada por:

$$
m^{2} \bar{B}^{2}[\hat{\phi}(\lambda), \hat{\theta}]=\sum_{a, b, c, d, e, f} d_{a b c} d_{d e f} b_{a b} b_{d e} b_{c f}, \quad a, b, c, d, e, f=\phi_{G J}, \theta
$$


onde $m$ é o numero de parâmetros, $b_{a b}, b_{d e}$ e $b_{c f}$ são os elementos da inversa da matriz de informação observada e $d_{a b c}$ e $d_{d e f}$ são as derivadas terceiras parciais de $l\left(\phi_{G J}, \theta\right)$, em relação a $\phi_{G J}$ e $\theta$, calculadas no ponto de máxima verossimilhança.

$\mathrm{O}$ que fazemos na prática é, calcular a medida de não normalidade para diversos valores de $\lambda$, escolhendo aquele para o qual $m^{2} \bar{B}^{2}[\hat{\phi}(\lambda), \hat{\theta}] \approx 0$.

O T-plot de Hills e Smith para a função de verossimilhança profile também deve ser obtido ponto a ponto através da relação:

$$
T\left(\phi_{G J}\right)=\operatorname{sgn}\left(\phi_{G J}-\hat{\phi}_{G J}\right)\left\{-2\left[l_{\hat{\theta}}\left(\phi_{G J}\right)-l\left(\hat{\phi}_{G J} ; \hat{\theta}\right)\right]\right\}
$$

\subsection{Análise Bayesiana.}

Apresentamos a seguir uma análise Bayesiana para a função de confiabilidade considerando uma densidade a priori não-informativa, assumindo dados com censuras de tipo II.

Uma densidade a priori não-informativa conjunta para $r$ e $\beta$ pode ser escrita por:

$$
p_{0}(r, \beta)=p_{0}(r / \beta) p_{0}(\beta)
$$

onde $p_{0}(\beta)$ é uma densidade a priori marginal para $\beta$ e a densidade a priori condicionada $p_{0}(r / \beta)$, determinada pela regra de Jeffreys, é dada por:

$$
p_{0}(r / \beta) \propto[\mathbf{I}(r / \beta)]^{1 / 2}
$$

onde $\mathbf{I}(r / \beta)=d /\left[(-\ln r)^{2} r^{2}\right]$ é a informação de Fisher condicionada em $\beta$. 
Desta forma, assumindo uma densidade a priori localmente uniforme para $\beta$, isto é, $p_{0}(\beta) \propto 1 / \beta$, a densidade a priori definida em (5.35) é dada por:

$$
p_{0}(r, \beta) \propto \frac{1}{\beta r(-\ln r)}, \quad 0 \leq r \leq 1, \beta>0
$$

Uma outra possibilidade seria assumir $\alpha$ e $\beta$, no modelo inicial, independentes. Assim, uma densidade a priori não-informativa conjunta para $\alpha$ e $\beta$ é dada por:

$$
p_{0}(\alpha, \beta)=p_{0}(\alpha) p_{0}(\beta)
$$

Tomando as densidades a priori marginais para $\alpha$ e $\beta$ localmente uniformes, $p_{0}(\alpha) \propto 1 / \alpha$ e $p_{0}(\beta) \propto 1 / \beta$, temos:

$$
p_{0}(\alpha, \beta)=\frac{1}{\alpha \beta}, \quad \alpha>0, \beta>0
$$

Portanto, uma densidade a priori conjunta para $r$ e $\beta$ pode ser obtida através de uma transformação de variáveis em $r=R\left(t_{0}\right)=\exp \left[-\left(t_{0} / \alpha\right)^{\beta}\right]$ e $\beta$, cujo Jacobiano é dado por:

$$
J=\left|\begin{array}{cc}
\partial \alpha / \partial r & \partial \beta / \partial r \\
\partial \alpha / \partial \beta & \partial \beta / \partial \beta
\end{array}\right|=\left|\begin{array}{cc}
t_{0}(-\ln r)^{-(1 / \beta+1)} / r \beta & \partial \beta / \partial r \\
0 & 1
\end{array}\right|
$$


O determinante de $J$, é dado por:

$$
|J|=\frac{t_{0}(-\ln r)^{-(1 / \beta+1)}}{r \beta}
$$

Desta forma, uma outra densidade a priori não-informativa conjunta para $r$ e $\beta$ é dada por:

$$
p_{0}(r, \beta) \propto \frac{1}{\beta^{2} r(-\ln r)}, \quad 0 \leq r \leq 1, \beta>0
$$

Como podemos observar as expressões (5.37) e (5.42) são bem parecidas, porém em nossos estudos vamos considerar apenas a densidade a priori não-informativa dada em (5.37).

A densidade a posteriori conjunta para $r$ e $\beta$, considerando a densidade a priori não-informativa obtida em (5.37) é dada por:

$$
p(r, \beta / \text { dados }) \propto \frac{\beta^{d-1} \prod_{i \in D} t_{(i)}^{\beta-1}}{t_{0}^{d \beta}}(-\ln r)^{d-1} r^{T(\beta) / r_{0}^{\beta}-1}, \quad 0 \leq r \leq 1, \quad \beta>0 .
$$

O valor esperado a posteriori da confiabilidade, $E(R /$ dados $)$, não pode ser obtido analiticamente, devendo ser determinado através de métodos numéricos ou de aproximação, como por exemplo o método de Laplace (ver apêndice D). 


\subsubsection{Reparametrização de Guerrero e Johnson para a Densidade a Posteriori.}

Considerando a transformação de Guerrero e Johnson para a função de confiabilidade, $\operatorname{com} \theta=\ln \beta$, a densidade a posteriori conjunta para $\phi_{G J}(\lambda)$ e $\beta$, assumindo a densidade a priori (5.37), é dada por:

$p\left(\phi_{G J}, \beta /\right.$ dados $) \propto \mathrm{e}^{d \theta} t_{0}^{-d \mathrm{e}^{\theta}}\left(\prod_{i \in D} t_{(i)}^{\mathrm{e}^{\theta}-1}\right)\left(1+\phi_{G J}\right)^{-(1 / \lambda+1)}\left[B\left(\phi_{G J}\right)\right]^{d-1}\left[1+A\left(\phi_{G J}\right)\right]^{-\left(T(\theta) / t_{0}^{e^{\theta}}+1\right)}$,

onde $-1 \leq \phi_{G J}<\infty, \quad-\infty<\theta<\infty$.

Novamente devemos recorrer a métodos de aproximação para obter a moda e a esperança a posteriori de (5.44).

\subsubsection{Análise Bayesiana com $\beta$ Conhecido.}

Quando o valor de $\beta$ é conhecido, a análise Bayesiana é bastante simplificada, uma vez que ficamos num caso especial da distribuição exponencial. Assim, a densidade a priori de Jeffreys para a função de confiabilidade (ver por exemplo, Martz $\&$ Waller, 1982), é dada por:

$$
p_{0}(r) \propto \frac{1}{r(-\ln r)}, \quad 0 \leq r \leq 1
$$

e, consequentemente, a densidade a posteriori para $r$ é dada por: 


$$
p(r / \text { dados })=\frac{\left(T / t_{0}^{\beta}\right)^{d}}{\Gamma(d)}(-\ln r)^{d-1} r^{T / t_{0}^{\beta}-1}, \quad 0 \leq r \leq 1,
$$

onde $T=\sum_{i=1}^{d} t_{(i)}^{\beta}+(n-d) t_{(d)}^{\beta}$, que como podemos notar é semelhante à obtida em (4.6).

$$
\begin{aligned}
& \text { A moda de (5.46), dada por } \tilde{r}=\exp \left\{-\frac{(d-1) t_{0}^{\beta}}{\left(T-t_{0}^{\beta}\right)}\right\}, \text { e sua esperança a posteriori: } \\
& \qquad E(R / \text { dados })=\left(1+\frac{t_{0}^{\beta}}{T}\right)^{-d}
\end{aligned}
$$

são estimadores Bayesianos para a função de confiabilidade.

Aplicando a reparametrização de Guerrero e Johnson dada em (2.3), com $\lambda$ obtido de (5.27), a densidade a posteriori para $\phi_{G J}(\lambda)$ é dada por:

$$
p\left(\phi_{G J} / \text { dados }\right)=\frac{\left(T / t_{0}^{\beta}\right)^{d}}{\lambda \Gamma(d)}\left[B\left(\phi_{G J}\right)\right]^{d-1}\left[1+A\left(\phi_{G J}\right)\right]^{-\left(T / t_{0}^{\beta}+1\right)}\left(1+\phi_{G J}\right)^{-(1+1 / \lambda)}
$$

com $-1 \leq \phi_{G J}<\infty$.

A moda e a esperança a posteriori de (5.48) devem ser obtidas através de métodos numéricos ou de aproximação, como por exemplo o de Laplace (ver apêndice D). 


\section{Capítulo 6}

\section{REPARAMETRIZAÇÃO PARA A CONFIABILIDADE EM TESTES ACELERAdOS CONSIDERANDO O MODELO DE LEI DE POTÊNCIA INVERSA.}

Neste capítulo exploramos a reparametrização de Guerrero \& Johnson (1982) para a confiabilidade em testes acelerados, considerando o modelo de Lei de Potência e tempos de vida com distribuição de Weibull.

Testes acelerados são de grande aplicação na indústria eletro-eletrônica onde, devido ao grande avanço tecnológico, exige-se componentes com baixa taxa de falha para se obter grande confiabilidade. Determinar essa confiabilidade através de experimentos nos níveis usuais de stress $^{55}$, ou seja, nas condições normais de uso, é quase impossível pois normalmente exigem um tempo muito longo ou custo muito elevado. Nestas situações, utiliza-se testes acelerados que consistem em experimentos onde as peças ou componentes são submetidas a níveis de stress mais altos do que o usual, reduzindo artificialmente seu tempo de vida. Através dos testes de sobrevivência conduzidos sob condições aceleradas, chamados "testes de sobrevivência acelerados", podemos fazer inferências sobre características da distribuição de vida correspondentes às condições normais de uso do componente.

Encontramos na literatura vários modelos para testes acelerados, dentre os quais os de Arrhenius, Eyring, Lei de Potência ou ainda um modelo geral introduzido por

\footnotetext{
5 Stress é um fator relacionado a um produto que afeta o seu tempo de vida, como por exemplo a voltagem, temperatura, pressão, ou outros fatores.
} 
Klein e Basu (1981) que inclui como casos particulares cada um dos modelos citados (ver Achcar e Louzada-Neto, 1992).

Seja uma variável aleatória $T$ denotando o tempo de vida de uma unidade com distribuição de Weibull dada por:

$$
f(t ; \alpha, \beta)=\frac{\beta}{\alpha}\left(\frac{t}{\alpha}\right)^{\beta-1} \exp \left\{-\left(\frac{t}{\alpha}\right)^{\beta}\right\}, \quad t \geq 0, \quad \alpha, \beta>0 .
$$

Considere, ainda, uma variável stress $V$ afetando o parâmetro de escala $\alpha$ mas com o mesmo parâmetro de forma $\beta$ para os seus diferentes níveis. Conforme o modelo de potência, com $k$ niveis de stress, temos:

$$
\alpha_{i}=\frac{\delta}{V_{i}^{\gamma}}, \quad \delta>0, \quad-\infty<\gamma<\infty \quad i=1,2, \ldots, k .
$$

\subsection{Inferências para a Confiabilidade de Sistemas com uma Unidade.}

Assumindo o modelo de potência com tempos de vida com distribuição de Weibull e considerando censuras tipo II, a função de verossimilhança para os parâmetros $\alpha_{1}, \ldots, \alpha_{k}$ e $\beta$ é dada por:

$$
\begin{aligned}
L\left(\alpha_{1}, \ldots, \alpha_{k}, \beta\right) & =\prod_{i=1}^{k} L\left(\alpha_{i}, \beta\right) \\
& =\frac{\beta^{d}}{\prod_{i=1}^{k} \alpha_{i}^{d_{i} \beta}}\left(\prod_{i=1}^{k} \prod_{j \in D_{i}} t_{i(j)}^{\beta-1}\right) \exp \left[-\sum_{i=1}^{k} \frac{T_{i}(\beta)}{\alpha_{i}^{\beta}}\right],
\end{aligned}
$$


onde: $d=\sum_{i=1}^{k} d_{i}, n_{i}$ e $d_{i}$ são, respectivamente, o número total de falhas, o tamanho da amostra e o número de falhas no $i$-ésimo nível de stress, $D_{i}$ é o conjunto de falhas no $i$ ésimo nível de stress e $T_{i}(\beta)=\sum_{j=1}^{d_{i}} t_{i(j)}^{\beta}+\left(n_{i}-d_{i}\right) t_{i\left(d_{i}\right)}^{\beta}$.

Portanto, de (6.3) e (6.2), a função de verossimilhança para os parâmetros $\beta, \gamma$ e $\delta$ é dada por:

$$
\begin{aligned}
L(\beta, \gamma, \delta) & =\frac{\beta^{d}}{\prod_{i=1}^{k}\left(\delta / V_{i}^{\gamma}\right)^{d_{j} \beta}}\left(\prod_{i=1}^{k} \prod_{j \in D_{i}} t_{i j}^{\beta-1}\right) \exp \left[-\sum_{i=1}^{k} \frac{T_{i}(\beta)}{\left(\delta / V_{i}^{\gamma}\right)^{\beta}}\right] \\
& =\frac{\beta^{d}}{\delta^{d \beta}}\left(\prod_{i=1}^{k} V_{i}^{d_{i} \beta \gamma}\right)\left(\prod_{i=1}^{k} \prod_{j \in D_{i}} t_{i(j)}^{\beta-1}\right) \exp \left[-\sum_{i=1}^{k} \frac{T_{i}(\beta) V_{i}^{\gamma \beta}}{\delta^{\beta}}\right]
\end{aligned}
$$

O logaritmo da função de verossimilhança é dado por:

$$
l(\beta, \gamma, \delta)=d \ln \beta-d \beta \ln \delta+\beta \gamma \sum_{i=1}^{k} d_{i} \ln V_{i}+(\beta-1) \sum_{i=1}^{k} \sum_{j \in D_{i}} \ln t_{i(j)}-\sum_{i=1}^{k} \frac{T_{i}(\beta) V_{i}^{\gamma \beta}}{\delta^{\beta}}
$$

Considerando um determinado nível de stress $p$, a função de confiabilidade de sistemas com uma unidade, num dado instante $t_{0}$, é dada por:

$$
R_{p}\left(t_{0}\right)=\exp \left[-\left(t_{0} / \alpha_{p}\right)^{\beta}\right], \quad t_{0} \geq 0
$$

Assim, de (6.2) e (6.6), temos,

$$
\delta=t_{0} V_{p}^{\gamma}\left(-\ln r_{p}\right)^{-1 / \beta}, \quad 0 \leq r_{p}=R_{p}\left(t_{0}\right) \leq 1
$$


Desta forma, a função de verossimilhança para $r_{p}, \gamma$ e $\beta$ é dada por:

$$
L\left(r_{p}, \gamma, \beta\right)=\frac{\beta^{d}\left(-\ln r_{p}\right)^{d}}{\left(t_{0} V_{p}^{\gamma}\right)^{d \beta}}\left(\prod_{i=1}^{k} V_{i}^{d_{i} \beta \gamma}\right)\left(\prod_{i=1}^{k} \prod_{j \in D_{i}} t_{i(j)}^{\beta-1}\right) \exp \left[\left(\ln r_{p}\right) \sum_{i=1}^{k} \frac{T_{i}(\beta) V_{i}^{\gamma \beta}}{t_{0}^{\beta} V_{p}^{\gamma \beta}}\right]
$$

e o logaritmo da função de verossimilhança, é dado por:

$$
\begin{aligned}
l\left(r_{p}, \gamma, \beta\right)= & d \ln \beta-d \beta \ln t_{0}+\beta \gamma K_{1}+(\beta-1) K_{2}+d \ln \left(-\ln r_{p}\right)+ \\
& +\left(\ln r_{p}\right) \sum_{i=1}^{k} T_{i}(\beta)\left[\frac{Q_{i}(\gamma)}{t_{0}}\right]^{\beta}
\end{aligned}
$$

onde $\left\{\begin{array}{c}K_{1}=\sum_{i=1}^{k} d_{i} \ln V_{i}-d \ln V_{p} \\ K_{2}=\sum_{i=1}^{k} \sum_{j \in D_{i}} \ln t_{i(j)} \\ Q_{i}(\gamma)=\left(V_{i} / V_{p}\right)^{\gamma}\end{array}\right.$

Com $\beta=1$, temos o modelo exponencial para os tempos de vida, e o logaritmo da função de verossimilhança para $r_{p}$ e $\gamma$ é dado por:

$$
l\left(r_{p}, \gamma\right)=-d \ln t_{0}+\gamma K_{1}+d \ln \left(-\ln r_{p}\right)+\frac{\left(\ln r_{p}\right)}{t_{0}} \sum_{i=1}^{k} T_{i} Q_{i}(\gamma)
$$

onde: $T_{i}=\sum_{j=1}^{d_{i}} t_{i(j)}+\left(n_{i}-d_{i}\right) t_{i\left(d_{i}\right)}$. 


\subsubsection{Estimadores de Máxima Verossimilhança.}

Os estimadores de máxima verossimilhança dos parâmetros $r_{p}, \gamma$ e $\beta$ são obtidos através das derivadas parciais:

$$
\begin{aligned}
& \frac{\partial l}{\partial \beta}=\frac{d}{\beta}-d \ln t_{0}+\gamma K_{1}+K_{2}+\left(\ln r_{p}\right) \sum_{i=1}^{k} T_{i}^{\prime}(\beta)\left(\frac{Q_{i}(\gamma)}{t_{0}}\right)^{\beta}+ \\
& +\left(\ln r_{p}\right) \sum_{i=1}^{k} T_{i}(\beta)\left(\frac{Q_{i}(\gamma)}{t_{0}}\right)^{\beta} \ln \left(\frac{Q_{i}(\gamma)}{t_{0}}\right) \\
& \frac{\partial l}{\partial \gamma}=\beta K_{1}+\beta\left(\ln r_{p}\right) \sum_{i=1}^{k} \frac{T_{i}(\beta)}{t_{0}^{\beta}}\left[Q_{i}(\gamma)\right]^{\beta-1} Q_{i}^{\prime}(\gamma) \\
& \frac{\partial l}{\partial r_{p}}=\frac{d}{\left(\ln r_{p}\right) r_{p}}+\frac{1}{r_{p}} \sum_{i=1}^{k} T_{i}(\beta)\left[\frac{Q_{i}(\gamma)}{t_{0}^{\beta}}\right]^{\beta}
\end{aligned}
$$

onde $\quad T_{i}(\beta)=\sum_{j=1}^{d_{i}} t_{i(j)}^{\beta} \ln t_{i(j)}+\left(n_{i}-d_{i}\right) t_{i\left(d_{i}\right)}^{\beta} \ln t_{i\left(d_{i}\right)}$ e $Q_{i}^{\prime}(\gamma)=\left(V_{i} / V_{p}\right)^{\gamma} \ln \left(V_{i} / V_{p}\right)$

Para isso devemos utilizar um método iterativo, por exemplo Newton-Raphson, e encontrar $\hat{r}_{p}, \hat{\gamma}$ e $\hat{\beta}$ tal que: $\partial l / \partial \beta=0, \partial l / \partial \gamma=0$ e $\partial l / \partial r_{p}=0$, sendo que $\hat{r}_{p}$ é dado pela seguinte relação:

$$
\hat{r}_{p}=\exp \left\{-d t_{0}^{\hat{\beta}} / \sum_{i=1}^{k} T_{i}(\hat{\beta})\left[Q_{i}(\hat{\gamma})\right]^{\hat{\beta}}\right\}
$$


Desta forma, reduzimos (6.11) a um sistema de duas equações.

Como já sabemos, os estimadores de máxima verossimilhança $\hat{r}_{p}, \hat{\gamma}$ e $\hat{\beta}$ têm normalidade assintótica dada por:

$$
\left(\hat{r}_{p}, \hat{\gamma}, \hat{\beta}\right) \stackrel{a}{\sim} N_{3}\left[\left(r_{p}, \gamma, \beta\right) ; \mathbf{I}_{0}^{-1}\right]
$$

onde $\mathbf{I}_{\mathbf{0}}$ é a matriz de informação observada dada por:

$$
\mathbf{I}_{\mathbf{0}}=\left[\begin{array}{ccc}
-\left.\frac{\partial^{2} l}{\partial \beta^{2}}\right|_{\hat{r}_{p}, \hat{\gamma}, \hat{\beta}} & -\left.\frac{\partial^{2} l}{\partial \beta \partial \gamma}\right|_{\hat{p}_{p}, \hat{\gamma}, \hat{\beta}} & -\left.\frac{\partial^{2} l}{\partial \beta \partial r_{p}}\right|_{\hat{r}_{p}, \hat{\gamma}, \hat{\beta}} \\
\text { simétrica } & -\left.\frac{\partial^{2} l}{\partial \gamma^{2}}\right|_{\hat{r}_{p}, \hat{\gamma}, \hat{\beta}} & -\left.\frac{\partial^{2} l}{\partial \beta \partial r_{p}}\right|_{\hat{r}_{p}, \hat{\gamma}, \hat{\beta}} \\
& & -\left.\frac{\partial^{2} l}{\partial r_{p}^{2}}\right|_{\hat{r}_{p}, \hat{\gamma}, \hat{\beta}}
\end{array}\right]
$$

Obs: $\partial^{2} l /\left.\partial r_{p}^{2}\right|_{\hat{r}_{p}, \hat{\gamma}, \hat{\beta}}=-d /\left(\ln \hat{r}_{p}\right)^{2} \hat{r}_{p}^{2}$

Intervalos de confiança para a confiabilidade devem ser obtidos por meio da normalidade assintótica do estimador de máxima verossimilhança, dada em (6.13), uma vez que não conseguimos encontrar uma distribuição exata para tal. Portanto um intervalo de confiança $(1-\alpha) \times 100 \%$ é dado por:

$$
\hat{r}_{p} \pm \sigma_{\hat{r}_{p}} Z_{\alpha / 2}
$$


onde: $\sigma_{\hat{r}_{p}}$ é a estimativa do desvio padrão da confiabilidade, obtido pelo elemento correspondente a $r_{p}$ na inversa da matriz de informação observada e $Z_{\alpha / 2}$ é o valor da distribuição $N(0,1)$ cujo quantil vale $\alpha / 2$.

\subsection{Caso especial $\beta=1$ : Distribuição Exponencial.}

Como caso especial de (6.1), temos a distribuição Exponencial para os tempos de vida quando $\beta=1$. Desta forma a função de verossimilhança com $r_{p}$ e $\gamma$ desconhecidos, é dada por:

$$
L\left(r_{p}, \gamma\right)=\frac{\left(-\ln r_{p}\right)^{d}}{\left(t_{0} V_{p}^{\gamma}\right)^{d}}\left(\prod_{i=1}^{k} V_{i}^{d_{i} \gamma}\right) r_{p}^{\sum_{i=1}^{k} T_{i} Q_{i}(\gamma) / t_{0}}
$$

e o logaritmo da função de verossimilhança é dado por:

$$
l\left(r_{p}, \gamma\right)=-d \ln t_{0}+\gamma K_{1}+d \ln \left(-\ln r_{p}\right)+\frac{\left(\ln r_{p}\right)}{t_{0}} \sum_{i=!}^{k} T_{i} Q_{i}(\gamma)
$$

onde $T_{i}=\sum_{j=1}^{d_{i}} t_{i(j)}+\left(n_{i}-d_{i}\right) t_{i\left(d_{i}\right)}, Q_{i}(\gamma)=\left(V_{i} / V_{p}\right)^{\gamma}$ e $K_{1}=\sum_{i=1}^{k} d_{i} \ln V_{i}-d \ln V_{p}$.

Assim, o estimador de máxima verossimilhança para $r_{p}$ é dado por:

$$
\hat{r}_{p}=\exp \left\{-d t_{0} / \sum_{i=1}^{k} T_{i} Q_{i}(\hat{\gamma})\right\}
$$


e o estimador de máxima verossimilhança para $\gamma$ é obtido através de um método iterativo conforme a seguinte relação:

$$
K_{1}+\frac{\left(\ln \hat{r}_{p}\right)}{t_{0}} \sum_{i=1}^{k} T_{i} Q_{i}^{\prime}(\hat{\gamma})=0
$$

Substituindo (6.18) em (6.19), podemos escrever:

$$
\frac{\sum_{i=1}^{k} d_{i} \ln V_{i}}{d}-\frac{\sum_{i=1}^{k} T_{i} V_{i}^{\hat{\gamma}} \ln \left(V_{i}\right)}{\sum_{i=1}^{k} T_{i} V_{i}^{\hat{\gamma}}}=0
$$

Os estimadores de máxima verossimilhança $\hat{r}_{p}$ e $\hat{\gamma}$ têm distribuição normal assintótica baseada na matriz de informação observada $\mathbf{I}_{0}$, dada por:

$$
\left(\hat{r}_{p}, \hat{\gamma}\right) \stackrel{a}{\sim} N_{2}\left[\left(r_{p}, \gamma\right) ; \mathbf{l}_{0}^{-1}\right],
$$

onde

$$
\mathbf{I}_{0}=\left[\begin{array}{cc}
\frac{d}{\left(\ln \hat{r}_{p}\right)^{2} \hat{r}_{p}^{2}} & \frac{K_{1}}{\left(\ln \hat{r}_{p}\right) \hat{r}_{p}} \\
\text { simétrica } & \frac{d \sum_{i=1}^{k} T_{i} V_{i}^{\hat{\gamma}} \ln ^{2}\left(V_{i} / V_{p}\right)}{\sum_{i=1}^{k} T_{i} V_{i}^{\hat{\gamma}}}
\end{array}\right]
$$




\subsubsection{Reparametrização de Guerrero e Johnson $\operatorname{com} \beta=1$.}

Aplicando a reparametrização de Guerrero e Johnson com a finalidade de melhorar a normalidade assintótica do estimador de máxima verossimilhança, a função de verossimilhança, em (6.16), é dada por:

$$
L\left(\phi_{G J_{p}}, \gamma\right)=\frac{\left[B\left(\phi_{G J_{p}}\right)\right]^{d}}{\left(t_{0} V_{p}^{\gamma}\right)^{d}}\left(\prod_{i=1}^{k} V_{i}^{d_{i} \gamma}\right)\left[1+A\left(\phi_{G J_{p}}\right)\right]^{-\sum_{i=1}^{k} T_{i} Q_{(\gamma)} / t_{0}}
$$

e o logaritmo da função de verossimilhança é dado por:

$$
l\left(\phi_{G J_{p}}, \gamma\right)=-d \ln t_{0}+\gamma K_{1}+d \ln \left[B\left(\phi_{G J_{p}}\right)\right]-\frac{B\left(\phi_{G J_{p}}\right)}{t_{0}} \sum_{i=1}^{k} T_{i} Q_{i}(\gamma)
$$

onde $B\left(\phi_{G J_{p}}\right)=\ln \left[1+A\left(\phi_{G J_{p}}\right)\right]$ e $A\left(\phi_{G J_{p}}\right)=\left(1+\phi_{G J_{p}}\right)^{-1 / \lambda_{p}}$.

O estimador de máxima verossimilhança para $\phi_{G J_{p}}$, obtido através da propriedade de invariância é dado por: $\hat{\phi}_{G J_{p}}=\left(\hat{r}_{p}^{-1}-1\right)^{-\lambda_{p}}-1$.

Conforme verificamos anteriormente, através da parametrização $\left(\phi_{G J_{p}}, \gamma\right)$ esperamos melhorar a normalidade assintótica dos estimadores de máxima verossimilhança, obtendo assim, inferências mais precisas para a função de confiabilidade $R\left(t_{0}\right)$. 
Para determinar o valor adequado de $\lambda$, podemos utilizar a medida de não normalidade de Kass e Slate , definida em (2.13), dada por:

$$
m^{2} \bar{B}^{2}\left[\hat{\phi}_{G J_{p}}(\lambda), \hat{\gamma}\right]=\sum_{a, b, c, d, e, f} d_{a b c} d_{d e f} b_{a b} b_{d e} b_{c f}, \quad a, b, c, d, e, f=\phi_{G J_{p}}, \gamma
$$

onde $m$ é o numero de parâmetros, $b_{a b}, b_{d e}$ e $b_{c f}$ são os elementos da inversa da matriz de informação observada e $d_{a b c}$ e $d_{d e f}$ são as derivadas terceiras parciais de $l\left(\phi_{G_{F}}, \gamma\right)$, em relação a $\phi_{G J_{p}}$ e $\gamma$, calculadas no ponto de máxima verossimilhança.

Para que possamos assegurar a normalidade dos parâmetros $\left(\phi_{G_{p}}, \gamma\right)$, devemos encontrar $\lambda$ tal que tenhamos $m^{2} \bar{B}^{2}\left[\hat{\phi}_{G_{p}}(\lambda), \hat{\gamma}\right] \approx 0$. Então, o que fazemos na prática é, calcular a medida de não normalidade para diversos valores de $\lambda$, escolhendo aquele para o qual $m^{2} \bar{B}^{2}\left[\hat{\phi}_{G J_{p}}(\lambda), \hat{\gamma}\right] \approx 0$.

Podemos também aplicar a medida $m^{2} \overline{\mathrm{B}}^{2}$ para avaliar a normalidade conjunta dos estimadores de máxima verossimilhança na parametrização original $\left(\hat{r}_{p}, \hat{\gamma}\right)$.

\subsubsection{Inferências para a Confiabilidade Considerando a Função de Verossimilhança "Profile".}

Com $\gamma$ desconhecido as inferências sobre a função de confiabilidade são mais complexas, uma vez que devemos estudar a normalidade conjunta dos dois parâmetros do nosso modelo. Para amenizar nossas dificuldades podemos, como alternativa, considerar a função de verossimilhança "profile" de $r_{p}$, dada por: $L_{\hat{\gamma}}\left(r_{p}\right)=L\left(r_{p}, \hat{\gamma}\right)$, onde $\hat{\gamma}$ maximiza a função de verossimilhança conjunta $L\left(r_{p}, \gamma\right)$ para cada valor de $r_{p}$. Desta forma, o logaritmo da função de verossimilhança "profile" é dado por: 


$$
l_{\hat{\gamma}}\left(r_{p}\right)=-d \ln t_{0}+\hat{\gamma} K_{1}+d \ln \left(-\ln r_{p}\right)+\frac{\left(\ln r_{p}\right)}{t_{0}} \sum_{i=1}^{k} T_{i} Q_{i}(\hat{\gamma})
$$

onde, para cada valor de $r_{p}, \hat{\gamma}$ é obtido de:

$$
\sum_{i=1}^{k} T_{i} Q_{i}^{\prime}(\hat{\gamma})=-\frac{t_{0} K_{1}}{\ln r_{p}}
$$

Medidas e diagnósticos para a normalidade:

i) Terceira derivada padronizada do logaritmo da verossimilhança:

$$
S T D\left(\hat{r}_{p}\right)=\left|l_{\hat{\gamma}}^{m}\left(\hat{r}_{p}\right)\left[-l_{\hat{\gamma}}^{m}\left(\hat{r}_{p}\right)\right]^{-3 / 2}\right|=\left|d^{-1 / 2}\left(3 \ln \hat{r}_{p}+2\right)\right|
$$

onde $l_{\hat{r}}^{\prime \prime}\left(\hat{r}_{p}\right)=-d /\left[\left(\ln \hat{r}_{p}\right)^{2} \hat{r}_{p}^{2}\right]$ e $l_{\hat{\gamma}}^{m}\left(\hat{r}_{p}\right)=3 d /\left[\left(\ln \hat{r}_{p}\right)^{2} \hat{r}_{p}^{3}\right]+2 d /\left[\left(\ln \hat{r}_{p}\right)^{3} \hat{r}_{p}^{3}\right]$.

ii) T-plot de Hills e Smith:

$$
T\left(\hat{r}_{p}\right)=\operatorname{sgn}\left(r_{p}-\hat{r}_{p}\right)\left[-2 l_{\hat{\gamma}}\left(r_{p}\right)+2 l\left(\hat{r}_{p}, \hat{\gamma}\right)\right]^{1 / 2} .
$$




\subsubsection{Reparametrização de Guerrero e Johnson para a Função de Verossimilhança "Profile".}

Na reparametrização de Guerrero e Johnson, o logaritmo da verossimilhança "profile" é dado por:

$$
l_{\hat{\gamma}}\left(\phi_{G J_{p}}\right)=-d \ln t_{0}+\hat{\gamma} K_{1}+d \ln B\left(\phi_{G J_{p}}\right)-\frac{B\left(\phi_{G J_{p}}\right)}{t_{0}} \sum_{i=1}^{k} T_{i} Q_{i}(\hat{\gamma})
$$

onde, para cada valor de $\phi_{G J_{p}}, \hat{\gamma}$ é obtido de:

$$
\sum_{i=1}^{k} T_{i} Q_{i}^{\prime}(\gamma)=\frac{t_{0} K_{1}}{B\left(\phi_{G J_{p}}\right)}
$$

Medidas e diagnósticos para a normálidade:

i) A terceira derivada padronizada do logaritmo da verossimilhança "profile" na reparametrização de Guerrero e Johnson, é dada por:

$$
\begin{aligned}
\operatorname{STD}\left(\hat{\phi}_{G J_{p}}\right) & =\left|l_{\hat{r}}^{m}\left(\hat{\phi}_{G J_{p}}\right)\left[-l_{\hat{r}}^{n}\left(\hat{\phi}_{G J_{p}}\right)\right]^{-3 / 2}\right| \\
& =\left|d^{-1 / 2}\left\{2-3 \frac{B^{\prime \prime}\left(\hat{\phi}_{G J_{p}}\right) B\left(\hat{\phi}_{G J_{p}}\right)}{\left[B\left(\hat{\phi}_{G J_{p}}\right)\right]^{2}}\right\}\right|
\end{aligned}
$$


onde $\left\{\begin{array}{l}\ln \left(\hat{\phi}_{G J_{p}}\right)=-d\left[B^{\prime}\left(\hat{\phi}_{G J_{p}}\right) / B\left(\hat{\phi}_{G J_{p}}\right)\right]^{2} \\ l_{\hat{\gamma}}^{m}\left(\hat{r}_{p}\right)=2 d\left[B^{\prime}\left(\hat{\phi}_{G J_{p}}\right) / B\left(\hat{\phi}_{G J_{p}}\right)\right]^{3}-3 d B^{\prime \prime}\left(\hat{\phi}_{G J_{p}}\right) B^{\prime}\left(\hat{\phi}_{G J_{p}}\right) /\left[B\left(\hat{\phi}_{G J_{p}}\right)\right]^{2}\end{array}\right.$

Desta forma, para conseguir boa normalidade para a função de verossimilhança "profile", devemos encontrar um valor adequado de $\lambda_{p}$ que minimize (6.32), ou seja, tal que:

$$
2-\frac{3 B^{\prime \prime}\left(\hat{\phi}_{G J_{p}}\right) B^{\prime}\left(\hat{\phi}_{G J_{p}}\right)}{\left[B\left(\hat{\phi}_{G J_{p}}\right)\right]^{2}} \approx 0 .
$$

Desenvolvendo a expressão acima, obtemos:

$$
g\left(\lambda_{p}\right)=B\left(\hat{\phi}_{G J_{p}}\right)\left\{\left(1+\lambda_{p}\right)\left[1+\left(1+\hat{\phi}_{G J_{p}}\right)^{-1 / \lambda_{p}}\right]-1\right\}-2 / 3 \approx 0
$$

Na prática devemos variar $\lambda_{p}$, encontrar $\hat{\phi}_{G_{p}}\left(\lambda_{p}\right)$, e escolher aquele que minimiza $g\left(\lambda_{p}\right)$, dada em (6.34).

ii) T-plot de Hills e Smith:

$$
T\left(\phi_{G J_{p}}\right)=\operatorname{sgn}\left(\phi_{G_{p}}-\hat{\phi}_{G J_{p}}\right)\left[-2 l_{\hat{\gamma}}\left(\phi_{G J_{p}}\right)+2 l\left(\hat{\phi}_{G J_{p}}, \hat{\gamma}\right)\right]^{1 / 2}
$$




\subsubsection{Inferências para a Confiabilidade com $\beta=1$ e $\gamma$ Conhecido.}

Quando o valor de $\gamma$ é conhecido, a função de verossimilhança para $r_{p}$ é bastante simplificada, tornando as inferências mais simples, pois o problema pode ser considerado como um caso especial do modelo exponencial estudado no capítulo 3. Desta forma, a função de verosimilhança para a função de confiabilidade é dada por:

$$
L\left(r_{p}\right) \propto\left(-\ln r_{p}\right)^{d} r_{p}^{T / t_{0}}, \quad 0 \leq r_{p} \leq 1
$$

onde $T=\sum_{i=1}^{k} T_{i} Q_{i}, \quad T_{i}=\sum_{j=1}^{d_{i}} t_{i(j)}+\left(n_{i}-d_{i}\right) t_{i\left(d_{i}\right)}, \quad Q_{i}=V_{i}^{\gamma} / V_{p}^{\gamma}$, e o logaritmo da função de verossimilhança é dado por:

$$
l\left(r_{p}\right) \propto d \ln \left(-\ln r_{p}\right)+\frac{T}{t_{0}} \ln r_{p}
$$

Assim o estimador de máxima verossimilhança para $r_{p}$, obtido através de $l^{\prime}\left(r_{p}\right)=0$, é dado por $\hat{r}_{p}=\mathrm{e}^{-d t_{0} / T}$.

Testes de hipóteses e intervalos de confiança para $r_{p}$, quando $\gamma$ é desconhecido, podem ser obtidos da distribuição exata:

$$
\frac{2\left(-\ln r_{p}\right) T}{t_{0}} \sim \chi_{2 d}^{2}
$$

onde $T=\sum_{i=1}^{k} T_{i} Q_{i}, T_{i}=\sum_{j=1}^{d_{i}} t_{i(j)}+\left(n_{i}-d_{i}\right) t_{i\left(d_{i}\right)}$ e $Q_{i}=V_{i}^{\gamma} / V_{p}^{\gamma}$ 
Desta forma, um intervalo de confiança $(1-\alpha) \times 100 \%$ para $r_{p}$ é dado por:

$$
\left(\exp \left\{-\frac{t_{0} \chi_{2 d, 1-\alpha / 2}^{2}}{2 T}\right\} ; \exp \left\{-\frac{t_{0} \chi_{2 d, \alpha / 2}^{2}}{2 T}\right\}\right)
$$

onde $\chi_{2 d, q}^{2}$ é o $q$-ésimo quantil da distribuição qui-quadrado com $2 d$ graus de liberdade.

Podemos, ainda, aproximar os intervalos de confiança considerando a normalidade assintótica do estimador de máxima verossimilhança, dada por:

$$
\hat{r}_{p} \stackrel{a}{\sim} N\left[r_{p} ; \frac{d t_{0}^{2} \mathrm{e}^{-2 d t_{0} / T}}{T^{2}}\right]
$$

\subsubsection{Reparametrização de Guerrero e Johnson para a Função de} Confiabilidade com $\beta=1$ e $\gamma$ Conhecido.

Considerando a reparametrização de Guerrero e Johnson para melhorar a normalidade em (6.40), a função de verossimilhança para $\phi_{G J_{p}}=\phi_{G J_{p}}\left(\lambda_{p}\right)$, é dada por:

$$
L\left(\phi_{G J_{p}}\right) \propto\left[B\left(\phi_{G J}\right)\right]^{d}\left[1+A\left(\phi_{G J_{p}}\right)\right]^{-T / t_{0}},
$$

onde $T=\sum_{i=1}^{k} T_{i} Q_{i}, T_{i}=\sum_{j=1}^{d_{i}} t_{i(j)}+\left(n_{i}-d_{i}\right) t_{i\left(d_{i}\right)}, Q_{i}=V_{i}^{\gamma} / V_{p}^{\gamma}$ e o logaritmo da função de verossimilhança é dado por:

$$
l\left(\phi_{G J_{p}}\right) \propto d \ln \left[B\left(\phi_{G J_{p}}\right)\right]-\frac{T}{t_{0}} B\left(\phi_{G J_{p}}\right)
$$


O estimador de máxima verossimilhança para $\phi_{G J_{p}}=\phi_{G J_{p}}\left(\lambda_{p}\right)$, dado por $\hat{\phi}_{G J_{p}}=\left(\mathrm{e}^{d t_{0} / T}-1\right)^{-\lambda_{p}}-1$, tem distribuição normal assintótica dada por:

$$
\hat{\phi}_{G J_{p}} \stackrel{a}{\sim} N\left[\phi_{G J_{p}} ; \frac{d \lambda_{p}^{2} t_{0}^{2} \mathrm{e}^{2 d t_{0} / T}}{T^{2}\left(\mathrm{e}^{d t_{0} / T}-1\right)^{2\left(\lambda_{p}+1\right)}}\right]
$$

\section{Terceira derivada padronizada .}

A terceira derivada padronizada de $l\left(\phi_{G_{p}}\right)$ calculada no ponto de máxima verossimilhança, onde $\phi_{G J_{p}}=\phi_{G J_{p}}\left(\lambda_{p}\right)$, é dada por:

$$
\operatorname{STD}\left(\hat{\phi}_{G J_{p}}\right)=\left|d^{-1 / 2}\left\{2-3 \frac{B^{\prime \prime}\left(\hat{\phi}_{G J_{p}}\right) B\left(\hat{\phi}_{G J_{p}}\right)}{\left[B^{\prime}\left(\hat{\phi}_{G J_{p}}\right)\right]^{2}}\right\}\right|
$$

Fazendo $S T D\left(\hat{\phi}_{G_{p}}\right)=0$, para encontrar o valor adequado de $\lambda_{p}$, temos:

$$
2-3 \frac{d t_{0}}{T}\left\{\left(1+\lambda_{p}\right)\left[1+\left(\mathrm{e}^{d t_{0} / T}-1\right)^{-1}\right]-1\right\}=0
$$

que nos fornece um valor para $\lambda_{p}$ dado por:

$$
\lambda_{p}=\left(\frac{2 T}{3 d t_{0}}+1\right)\left(1-\mathrm{e}^{-d t_{0} / T}\right)-1
$$


que é a mesma expressão obtida anteriormente em (3.19), mas agora com $T=\sum_{i=1}^{k} T_{i} Q_{i}$ onde $T_{i}=\sum_{j=1}^{d_{i}} t_{i(j)}+\left(n_{i}-d_{i}\right) t_{i\left(d_{i}\right)}$ e $Q_{i}=V_{i}^{\gamma} / V_{p}^{\gamma}$

Com o valor de $\lambda_{p}$ obtido, esperamos encontrar inferências mais precisas para a função de confiabilidade.

\subsection{Análise Bayesiana Considerando $\beta=1$ e $\gamma$ Desconhecido.}

Nesta seção, sob o enfoque Bayesiano, aplicamos a reparametrização de Guerrero e Johnson visando melhorar a normalidade da densidade a posteriori conjunta de $\left(r_{p}, \gamma\right)$, considerando uma densidade a priori não não-informativa obtida pela regra de Jeffreys. A densidade a priori de Jeffreys, conforme seção 4.3, é dada por:

$$
p_{0}\left(r_{p}, \gamma\right) \propto\left[\operatorname{det} \mathbf{I}\left(r_{p}, \gamma\right)\right]^{1 / 2}
$$

onde $\mathbf{I}\left(r_{p}, \gamma\right)$ é a matriz de informação de Fisher (ver Box e Tiao, 1973).

Os elementos da matriz de informação de Fisher para o modelo de lei de potência $\operatorname{com} \beta=1$ e censuras de tipo II são dados por:

$$
\begin{aligned}
& E\left(-\frac{\partial^{2} l}{\partial r_{p}^{2}}\right)=\frac{d}{\left(-\ln r_{p}\right)^{2} r_{p}^{2}} \\
& E\left(-\frac{\partial^{2} l}{\partial r_{p} \partial \gamma}\right)=-\frac{\sum_{i=1}^{k} d_{i} \ln \left(V_{i} / V_{p}\right)}{\left(-\ln r_{p}\right) r_{p}}
\end{aligned}
$$




$$
E\left(-\frac{\partial^{2} l}{\partial^{2} \gamma}\right)=\sum_{i=1}^{k} d_{i} \ln ^{2}\left(V_{i} / V_{p}\right)
$$

Desta forma, o determinante da matriz de Informação de Fisher é dado por:

$$
\operatorname{det} \mathbf{I}\left(r_{p}, \gamma\right)=\frac{d \sum_{i=1}^{k} d_{i} \ln ^{2}\left(V_{i} / V_{p}\right)-\left[\sum_{i=1}^{k} d_{i} \ln \left(V_{i} / V_{p}\right)\right]^{2}}{\left(-\ln r_{p}\right)^{2} r_{p}^{2}}
$$

Como o termo no numerador de (6.49) é constante, então a densidade a densidade a priori conjunta para $\left(r_{p}, \gamma\right)$, segundo a regra de Jeffreys, é dada por:

$$
p_{0}\left(r_{p}, \gamma\right) \propto \frac{1}{\left(-\ln r_{p}\right) r_{p}}, \quad 0 \leq r_{p} \leq 1, \quad-\infty<\gamma<\infty
$$

Portanto, a densidade a posteriori conjunta para $\left(r_{p}, \gamma\right)$, considerando a densidade a priori obtida em (6.50), é dada por:

$$
p\left(r_{p}, \gamma / \text { dados }\right) \propto \frac{\Pi_{i=1}^{k} V_{i}^{\gamma d_{i}}}{V_{p}^{\gamma d}}\left(-\ln r_{p}\right)^{d-1} r_{p}^{\sum_{i=1}^{k} T_{i} Q_{i}(\gamma) / t_{0}-1}
$$

onde $0 \leq r_{p} \leq 1,-\infty<\gamma<\infty$.

Um estimador Bayesiano para a função de confiabilidade é dado pela sua esperança a posteriori, que neste caso não pode ser obtida analiticamente, forçando-nos a recorrer a métodos numéricos ou de aproximação de integrais (p. ex. Laplace, ver apêndice D). 


\subsubsection{Reparametrização de Guerrero e Jonhson para a Densidade a Posteriori.}

Com a reparametrização de Guerrero e Johnson, a densidade a posteriori conjunta para $\left(\phi_{G J_{p}}, \gamma\right)$, obtida através de transformação de variáveis, é dada por:

$p\left(\phi_{G J_{p}}, \gamma /\right.$ dados $) \propto \frac{\prod_{i=1}^{k} V_{i}^{\gamma d_{i}}}{\lambda_{p} V_{p}^{\gamma d}}\left(1+\phi_{G J_{p}}\right)^{-\left(1+1 / \lambda_{p}\right)}\left[B\left(\phi_{G J_{p}}\right)\right]^{d-1}\left[1+A\left(\phi_{G J_{p}}\right)\right]^{-\left(\sum_{i=1}^{k}{ }_{T}^{T} Q_{i}(\gamma) / t_{0}+1\right)}$,

com $-1 \leq \phi_{G J_{p}}<\infty, \quad-\infty<\gamma<\infty$.

A esperança a posteriori para a função de confiabilidade na nova parametrização é obtida pela seguinte relação:

$$
E_{\phi_{G J_{p}}}\left(R_{p} / \text { dados }\right)=E_{\phi_{G_{p}}}\left\{\left[1+A\left(\phi_{G J_{p}}\right)\right]^{-1} / \text { dados }\right\}
$$

que também não apresenta solução analítica, devendo, então, ser aproximada por métodos numéricos ou de aproximação de integrais.

\subsubsection{Análise Bayesiana com $\beta=1$ e $\gamma$ Conhecido.}

Para o caso em que o parâmetro $\gamma$ é conhecido, a densidade a priori de Jeffreys para $r_{p}$ tem a mesma forma que a obtida em (6.50), ou seja, 
$p_{0}\left(r_{p}\right) \propto 1 /\left(-\ln r_{p}\right) r_{p}, 0 \leq r_{p} \leq 1$. Assim, a densidade a posteriori para a função de confiabilidade quando $\gamma$ é conhecido é dada por:

$$
p\left(r_{p} / \text { dados }\right)=\frac{\left(T / t_{0}\right)^{d}}{\Gamma(d)}\left(-\ln r_{p}\right)^{d-1} r_{p}^{T / t_{0}-1}, \quad 0 \leq r_{p} \leq 1
$$

onde $T=\sum_{i=1}^{k} T_{i} Q_{i}, Q_{i}=V_{i}^{\gamma} / V_{p}^{\gamma}$ e $T_{i}=\sum_{j=1}^{d_{i}} t_{i(j)}+\left(n_{i}-d_{i}\right) t_{i\left(d_{i}\right)}$ que é uma densidade loggama negativa com moda dada por $\tilde{r}_{p}=\exp \left\{-\frac{(d-1) t_{0}}{\left(T-t_{0}\right)}\right\}$ e valor esperado dado por:

$$
E_{R_{p}}\left(R_{p} / \text { dados }\right)=\left(1+\frac{t_{0}}{T}\right)^{-d}
$$

$\mathrm{Na}$ reparametrização de Guerrero e Jonhson a densidade a posteriori para $\phi_{G J_{p}}=\phi_{G J_{p}}\left(\lambda_{p}\right)$, com $\gamma$ conhecido, é dada por:

$$
p\left(\phi_{G J} / \text { dados }\right)=\frac{\left(T / t_{0}\right)^{d}}{\lambda_{p} \Gamma(d)}\left(1+\phi_{G J}\right)^{-\left(1 / \lambda_{,}+1\right)}\left[B\left(\phi_{G J}\right)\right]^{d-1}\left[1+A\left(\phi_{G J}\right)\right]^{-\left(T / t_{t}+1\right)}
$$

$$
\text { onde }-1 \leq \phi_{G J_{p}}<\infty \text { e } T=\sum_{i=1}^{k} T_{i} Q_{i}, Q_{i}=V_{i}^{\gamma} / V_{p}^{\gamma} \text { e } T_{i}=\sum_{j=1}^{d_{i}} t_{i(j)}+\left(n_{i}-d_{i}\right) t_{i\left(d_{i}\right)} \text {. }
$$




\section{Capítulo 7}

\section{EXEMPLOS}

\subsection{Exemplo para um Sistema com uma Unidade, Considerando o Modelo Exponencial.}

\section{Análise Clássica.}

Na tabela 1, temos dados de um experimento de confiabilidade com uma amostra aleatória com $n=12$ unidades (dados introduzidos por Lawless (1982), p. 103). Os $n$ componentes foram colocados em teste, sendo observados os tempos de vida em horas até a ocorrência de $d=8$ falhas. Desta forma, o tempo total em teste é $T=\sum_{i=1}^{8} t_{(i)}+4 t_{(8)}=5063$ horas. Assumindo que estamos interessados na confiabilidade do sistema no instante $t_{0}=5$ horas, considerando o modelo exponencial para os tempos de vida, os estimadores de máxima verossimilhança (e.m.v.) para $\theta \mathrm{e}$ para a função de confiabilidade $R(5)$, são dados respectivamente por:

$$
\begin{aligned}
& \hat{\theta}=\frac{T}{d}=\frac{5063}{8}=632.875 \text { horas, } \mathrm{e} \\
& \hat{R}(5)=\mathrm{e}^{-5 / \hat{\theta}}=0.9921 .
\end{aligned}
$$


Usando a aproximação normal para $\hat{R}(5)$, um intervalo de confiança $95 \%$ para $R(5)$, obtido de $(3.12)$ é dado por $(0.9867 ; 0.9976)$.

Observando que $2 T / \theta$ tem uma distribuição qui-quadrado com $2 d$ graus de liberdade, um intervalo $95 \%$ exato para $\theta$, obtido de (3.5), é dado por ( $351.6 ; 1465.4$ ), que corresponde a um intervalo de confiança $95 \%$ para $R(5)$ dado por $(0.9859 ; 0.9966)$.

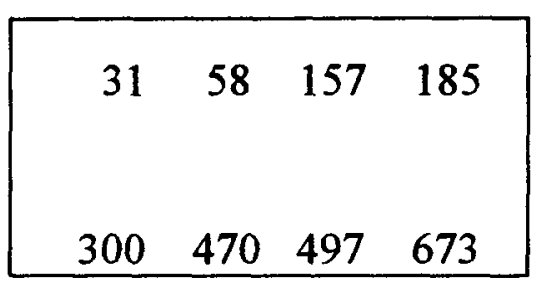

Tabela 1: Tempos de vida (em horas) dos 8 primeiros componentes a falharem.

Considerando a reparametrização de Guerrero e Johnson em (2.3), o valor de $\lambda$ obtido de (3.19) é dado por $\lambda=-0.3281$. Desta forma, o e.m.v. para $\phi_{G J}(-0.3281)$ é dado por:

$$
\hat{\phi}_{G J}(-0.3281)=\left[\frac{\hat{R}(5)}{1-\hat{R}(5)}\right]^{-0.3281}-1=-0.7955
$$

Da distribuição normal assintótica de $\hat{\phi}_{G J}(-0.3281)$, dada em (3.20), um intervalo de confiança $95 \%$ para $\phi_{G J}(-0.3281)$ é dado por $(-0.8422 ;-0.7487)$, que corresponde a um intervalo de confiança $95 \%$ para $R(5)$ dado por $(0.9854 ; 0.9964)$.

Comparando os intervalos de confiança para $R(5)$ obtidos pela normalidade assintótica dos estimadores de máxima verossimilhança com o intervalo obtido pela 
distribuição qui-quadrado, podemos observar que o intervalo de confiança na parametrização de Guerrero e Johnson é mais preciso que o intervalo de confiança assintótico baseado na parametrização original.

A fim de melhorar a normalidade da função de verossimilhança, também podemos considerar a parametrização de Aranda-Ordaz. Para $t_{0}=5$ horas, usando o método iterativo de Newton-Raphson para resolver (3.27), encontramos $\lambda=0.4246$ e o e.m.v. para $\phi_{A O}(0.4246)$ é dado por $\hat{\phi}_{A O}(0.4246)=0.7726$.

Através da aproximação normal do e.m.v., um intervalo de confiança $95 \%$ para $\phi_{A O}(0.4246)$ é dado por $(0.7136 ; 0.8322)$ que corresponde a um intervalo de confiança $95 \%$ para $R(5)$ dado por $(0.9854 ; 0.9964)$.

$\mathrm{Na}$ tabela 2 apresentamos intervalos de confiança $95 \%$ para $R\left(t_{0}\right)$, exatos e aproximados, com $t_{0}=5,30,500$ e 2000 horas, onde podemos avaliar os bons resultados obtidos para as inferências considerando as transformações $\phi_{G J}(\lambda)$ e $\phi_{A O}(\lambda)$, com os valores apropriados de $\lambda$. Através dos resultados vemos nitidamente que os intervalos nas parametrizações $\phi_{G J}(\lambda)$ e $\phi_{A O}(\lambda)$ estão mais próximos daqueles obtidos pela distribuição exata $\chi_{2 d}^{2}$, especialmente para os valores extremos de $t_{0}$, como é o caso de $t_{0}=500$ e 2000 horas.

Para verificar a normalidade da função de verossimilhança usamos o T-Plot de Hills e Smith. Assim, de (2.11) e (3.10) temos:

$$
T(r)=\operatorname{sgn}(r-\hat{r})\left\{-2\left[\frac{T}{t_{0}} \ln r+d \ln (-\ln r)-d \ln t_{0}-\ln \hat{r}\right]\right\}^{1 / 2} .
$$


Então, para $t_{0}=5$, temos:

$$
T(r)=\operatorname{sgn}(r-0.9921)\{-2025.2 \ln r-16 \ln (-\ln r)-93.4534\}^{1 / 2} .
$$

Considerando a reparametrização de Guerrero e Johnson, o T-Plot obtido de (2.11) e (3.14), é dado por:

$$
\begin{aligned}
T\left(\phi_{G J}\right)= & \operatorname{sgn}\left(\phi_{G J}+0.7955\right)\left\{2025.2 \ln \left[1+\left(1+\phi_{G J}\right)^{3.0479}\right]-\right. \\
& \left.-16 \ln \left\{\ln \left[1+\left(1+\phi_{G J}\right)^{3.0479}\right]\right\}-93.4534\right\}^{1 / 2}
\end{aligned}
$$

\begin{tabular}{|c|c|c|c|c|c|c|}
\hline$t_{0}$ & $\begin{array}{c}\text { distribuição } \\
\chi_{(2 d)}^{2} \text { para } \\
2 T / \theta\end{array}$ & $\begin{array}{c}\text { Normalidade } \\
\text { assintótica } \\
\text { para } \hat{R}\left(t_{0}\right)\end{array}$ & $\begin{array}{l}\lambda \text { dado } \\
\text { por } \\
(3.19)\end{array}$ & $\begin{array}{c}\text { Normalidade } \\
\text { assintótica } \\
\text { para } \hat{\phi}_{G J}(\lambda)\end{array}$ & $\begin{array}{c}\lambda \text { dado } \\
\text { por } \\
(3.27)\end{array}$ & $\begin{array}{c}\text { Normalidade } \\
\text { assintótica } \\
\text { para } \hat{\phi}_{A O}(\lambda)\end{array}$ \\
\hline 5 & $(0.9859 ; 0.9966)$ & $(0.9867 ; 0.9976)$ & -0.3281 & $(0.9854 ; 0.9964)$ & 0.4246 & $(0.9854 ; 0.9964)$ \\
\hline 30 & $(0.9182 ; 0.9797)$ & $(0.9224 ; 0.9850)$ & -0.3026 & $(0.9155 ; 0.9786)$ & 0.4844 & $(0.9152 ; 0.9788)$ \\
\hline 500 & $(0.2412 ; 0.7109)$ & $(0.2054 ; 0.7023)$ & 0.0071 & $(0.2332 ; 0.6930)$ & 0.2761 & $(0.2319 ; 0.6942)$ \\
\hline 2000 & $(0.0034 ; 0.2554)$ & $(-0.0505 ; 0.1353)$ & 0.1596 & $(0.0026 ; 0.2373)$ & 0.3339 & $(0.0021 ; 0.2477)$ \\
\hline
\end{tabular}

Na parametrização de Aranda-Ordaz, a expressão para o T-plot é semelhante às obtidas nas parametrizações anteriores.

Tabela 2: Intervalos de Confiança $95 \%$ para $R\left(t_{0}\right)$. 
Nas figuras 5, 6 e 7 apresentamos os gráficos T-Plot de Hills e Smith para o logaritmo da função de verossimilhança, nas parametrizações $R\left(t_{0}\right), \phi_{G J}(\lambda)$ e $\phi_{A O}(\lambda)$, considerando $t_{0}=5,500$ e 2000 horas, respectivamente. Como podemos observar, $T\left(\phi_{G J}\right)$ e $T\left(\phi_{A O}\right)$ apresentam boa linearidade, indicando, portanto, boa normalidade para suas respectivas funções de verossimilhança. Já na parametrização original, $T(r)$ é marcadamente curvilíneo, indicando a não-normalidade da função de verossimilhança.

Já, nas figuras 8, 9, e 10 temos os gráficos das funções de verossimilhança, nas diferentes parametrizações, considerando $t_{0}=5,500 \mathrm{e} 2000$ horas. Observamos que, para valores extremos de $R\left(t_{0}\right)$, as reparametrizações $\phi_{G J}(\lambda)$ e $\phi_{A O}(\lambda)$, com os valores apropriados de $\lambda$, melhoraram significativamente a normalidade da função de verossimilhança.
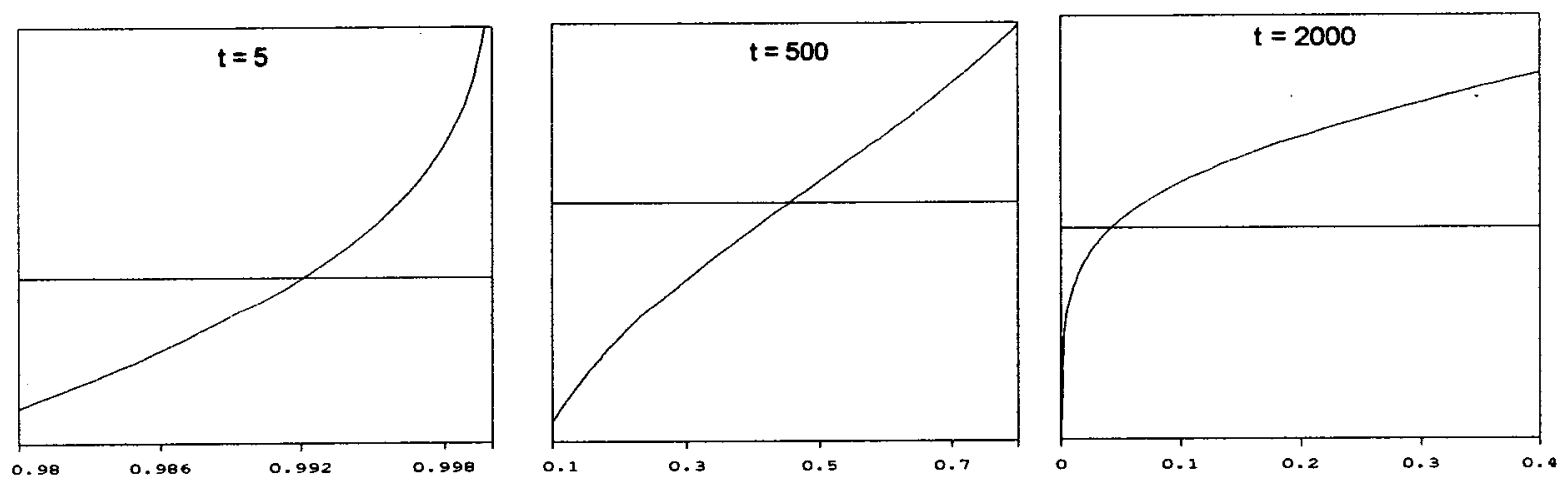

Figura 5: T-Plot de Hills e Smith $T(r)$, para $t_{0}=5,500$ e 2000 horas, respectivamente. 

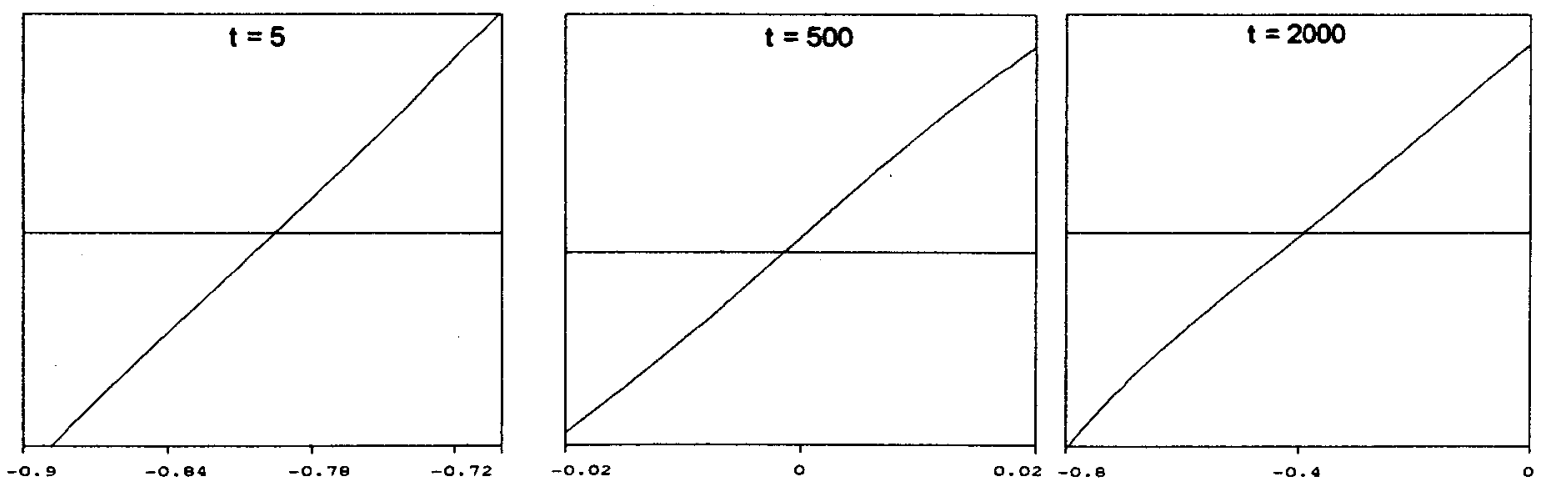

Figura 6: T-Plot de Hills e Smith $T\left(\phi_{G J}\right)$, para $t_{0}=5,500$ e 2000 horas, respectivamente.
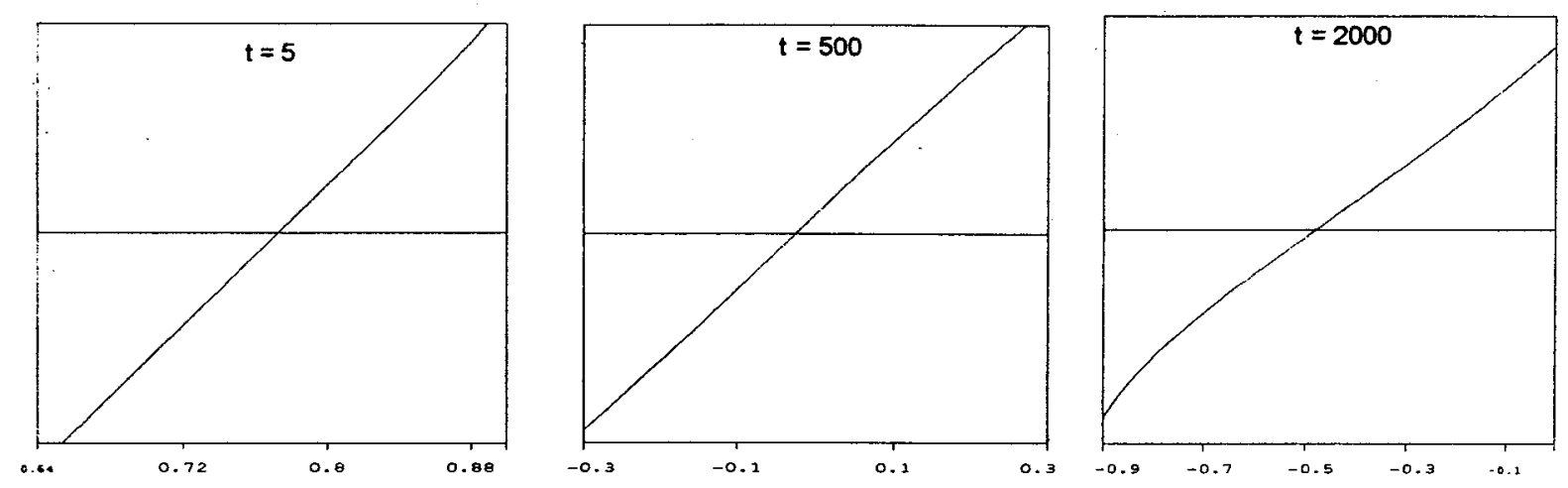

Figura 7: T-Plot de Hills e Smith $T\left(\phi_{A O}\right)$, para $t_{0}=5,500$ e 2000 horas, respectivamente.
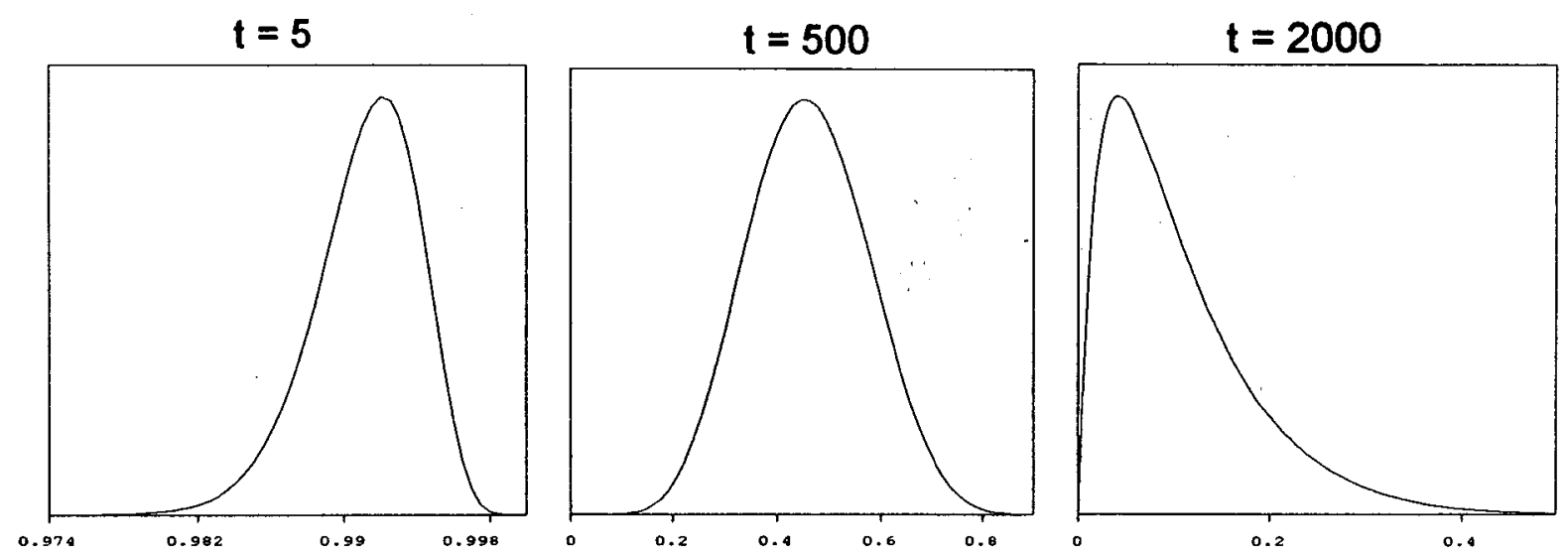

Figura 8: Verossimilhanças $L(r)$, para $t_{0}=5,500$ e 2000 horas, respectivamente. 

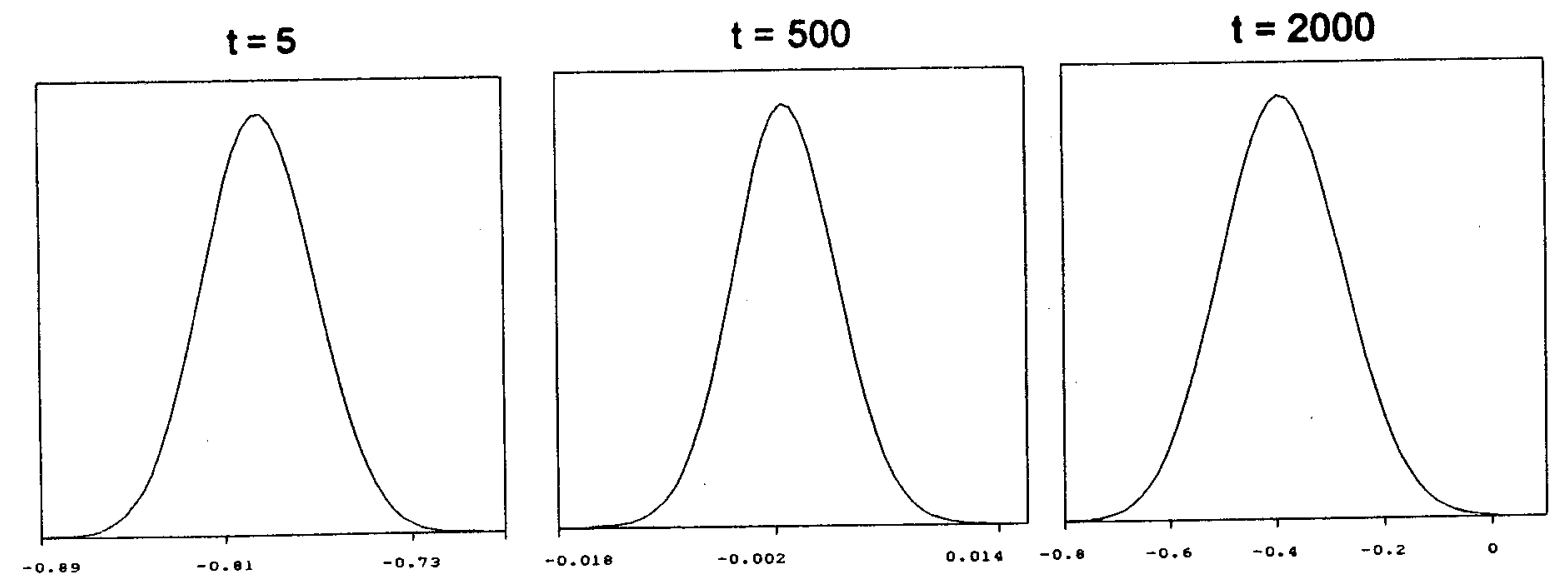

Figura 9: Verossimilhanças $L\left(\phi_{G J}\right)$, para $t_{0}=5,500$ e 2000 horas, respectivamente.
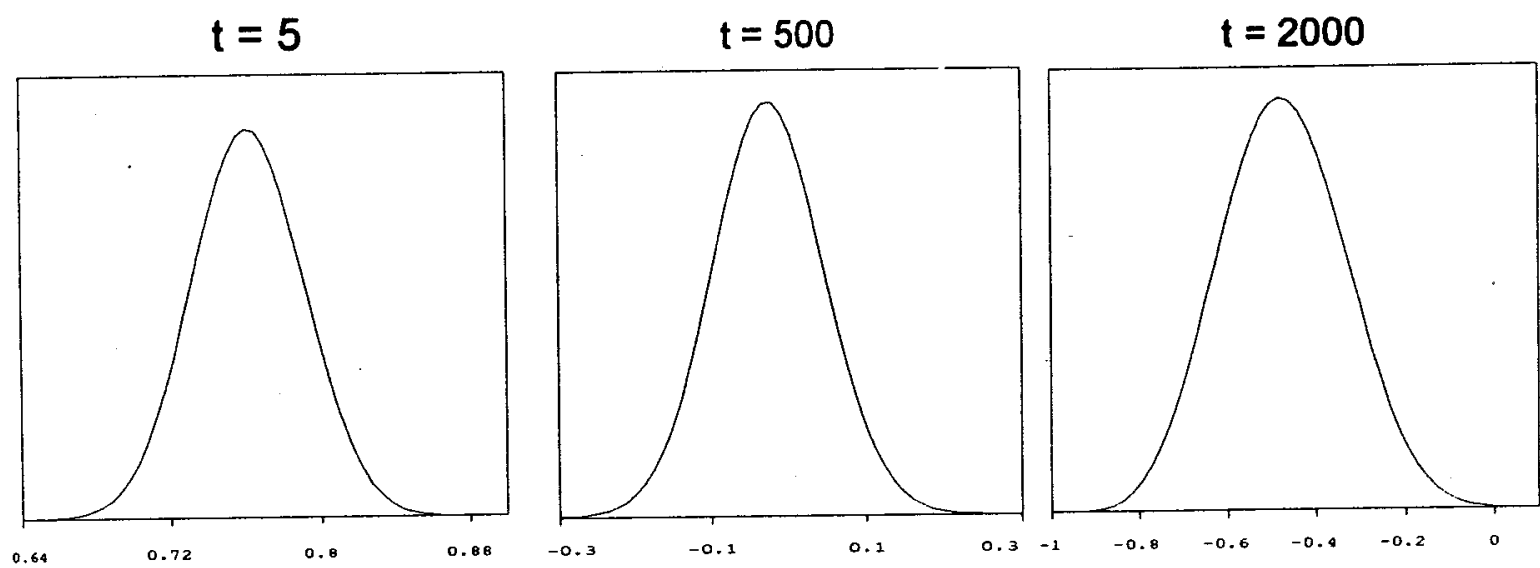

Figura 10: Verossimilhanças $L\left(\phi_{A O}\right)$, para $t_{0}=5,500$ e 2000 horas, respectivamente.

\section{Análise Bayesiana.}

Para um sistema com uma unidade, considerando uma densidade a priori nãoinformativa de Jeffreys, $p_{0}(r) \propto 1 /[(-\ln r) r]$, e com os dados da tabela 1 , a densidade a posteriori para a confiabilidade num instante $t_{0}=5$ é dada por:

$$
p_{1}(r / \text { dados }) \propto(-\ln r)^{7} r^{10116}, \quad 0 \leq r \leq 1 .
$$


A moda de (7.6) é dada por $\tilde{R}_{1}=0.9931 \mathrm{e}$, considerando a função de perda quadrática dada em (4.10), uma outra estimativa Bayesiana para $R(5)$, obtida de (4.11), é dada por $E(R /$ dados $)=0.9921$.

Considerando a transformação de Guerrero e Johnson, com $\lambda=-0.3281$, a densidade a posteriori para $\phi_{G J}$, apresentada em (4.13), é dada por:

$$
\begin{gathered}
p_{1}\left(\phi_{G J} / \text { dados }\right) \propto\left\{\ln \left[1+\left(1+\phi_{G J}\right)^{3.0479}\right]\right\}^{7}\left[1+\left(1+\phi_{G J}\right)^{3.0479}\right]^{-10136} \\
\left(1+\phi_{G J}\right)^{-2.0479}, \quad-1 \leq \phi_{G J} \leq \infty
\end{gathered}
$$

Da relação dada em (2.4), uma estimativa Bayesiana com relação à função de perda quadrática para $R(5)$, através da parametrização $\phi_{G J}$, é dada por:

$$
E_{\phi_{G J}}(R / \text { dados })=E_{\phi_{G J}}\left\{\left[1+\left(1+\phi_{G J}\right)^{-1 / \lambda}\right]^{-1} / \text { dados }\right\}
$$

Para resolver (7.8), aplicamos o método de Laplace para aproximação de integrais (ver apêndice $\mathrm{D}$ ), obtendo $E_{\phi_{G !}}(R /$ dados) $=0.9921$.

$\mathrm{Na}$ reparametrização de Aranda-Ordaz, $\operatorname{com} \lambda=0.4246$, aplicando o método de Laplace para aproximação de integrais, encontramos $E_{\phi_{A O}}(R /$ dados $)=0.9921$.

Considerando uma densidade a priori Uniforme, a densidade a posteriori para a confiabilidade no tempo $t_{0}=5$, obtida de (4.17), é dada por:

$$
p_{2}(r / \text { dados }) \propto(-\ln r)^{8} r^{1012.6}, \quad 0 \leq r \leq 1 .
$$


A moda de (7.9) é $\tilde{R}_{2}(5)=0.9921$ e a esperança a posteriori, obtida de (4.18), é dada por $E_{R}(R /$ dados $)=0.9912$.

Estimativas Bayesianas para $R(5)$, obtidas pelas esperanças a posteriori nas parametrizações de Guerrero e Johnson e Aranda-Ordaz, calculadas pelo método de Laplace são dadas, respectivamente, por $E_{\phi_{G}}(R /$ dados $)=0.9912 \quad \mathrm{e}$ $E_{\phi_{A O}}(R /$ dados $)=0.9912$.

Nas tabelas 3 e 4 apresentamos as estimativas de $R\left(t_{0}\right)$ e os erros relativos em \%, para $t_{0}=5,30,500$ e 2000 horas, considerando as densidades a priori de Jeffreys e uniforme, respectivamente. Comparando os valores encontrados, podemos verificar claramente que as parametrizações de $\phi_{G J}(\lambda)$ e $\phi_{A O}(\lambda)$ melhoraram sensivelmente a precisão das estimativas, especialmente para os valores extremos de $R\left(t_{0}\right)$, reduzindo significativamente o erro relativo.

\begin{tabular}{|c|c|cc|cc|cc|}
\hline \multirow{2}{*}{$t_{0}$} & \multicolumn{6}{|c|}{$E\left[R\left(t_{0}\right) /\right.$ dados $]$ aproximada pelo método de Laplace } \\
\cline { 2 - 8 } & Dada por & & Erro rel. & & Erro rel. & \multicolumn{3}{c|}{ Erro rel. } \\
& $(4.8)$ & $R\left(t_{0}\right)$ & $\%$ & $\phi_{G J}(\lambda)$ & $\%$ & $\phi_{A O}(\lambda)$ & $\%$ \\
\hline \hline \multirow{2}{*}{5} & 0.9921 & 0.9931 & 0.101 & 0.9921 & 0.000 & 0.9921 & 0.000 \\
30 & 0.9538 & 0.9595 & 0.598 & 0.9538 & 0.000 & 0.9538 & 0.000 \\
500 & 0.4708 & 0.5210 & 10.663 & 0.4710 & 0.042 & 0.4709 & 0.021 \\
2000 & 0.0697 & 0.1804 & 158.824 & 0.0687 & 1.435 & 0.0677 & $2 . .869$ \\
\hline
\end{tabular}

Tabela 3: Esperanças a Posteriori nas parametrizações $R\left(t_{0}\right), \phi_{G J}$ e $\phi_{A O}$ considerando uma densidade a priori de Jeffreys, comparadas com o valor exato (erros relativos em \%). 


\begin{tabular}{|c|c|c|c|c|c|c|c|}
\hline \multirow{3}{*}{$t_{0}$} & \multicolumn{7}{|c|}{$E\left[R\left(t_{0}\right) /\right.$ dados $]$ aproximada pelo método de Laplace } \\
\hline & Dada por & & Erro rel. & & Erro rel. & & Erro rel. \\
\hline & & $R\left(t_{0}\right)$ & $\%$ & $\phi_{G J}(\lambda)$ & $\%$ & $\phi_{A O}(\lambda)$ & $\%$ \\
\hline 5 & 0.9912 & 0.9912 & 0.000 & 0.9912 & 0.000 & 0.9912 & 0.000 \\
\hline 30 & 0.9485 & 0.9485 & 0.000 & 0.9485 & 0.000 & 0.9485 & 0.000 \\
\hline 500 & 0.4609 & 0.4600 & 0.195 & 0.4611 & 0.043 & 0.4610 & 0.022 \\
\hline 2000 & 0.1060 & 0.1223 & 15.377 & 0.1053 & 0.660 & 0.1047 & 1.226 \\
\hline
\end{tabular}

Tabela 4: Esperanças a Posteriori nas parametrizações $R\left(t_{0}\right), \phi_{G J}$ e $\phi_{A O}$, considerando uma densidade a priori Uniforme, comparadas com com o valor exato (erros relativos em \%).

Nas figuras 11 a 16 , temos os gráficos das densidades a posteriori para $R\left(t_{0}\right), \phi_{G J}(\lambda)$ e $\phi_{A O}(\lambda)$, considerando $t_{0}=5,500$ e 2000 horas, onde observamos a eficiência dạs parametrizações $\phi_{G J}(\lambda)$ e $\phi_{A O}(\lambda)$ na melhoria da normalidade das densidades a posteriori.
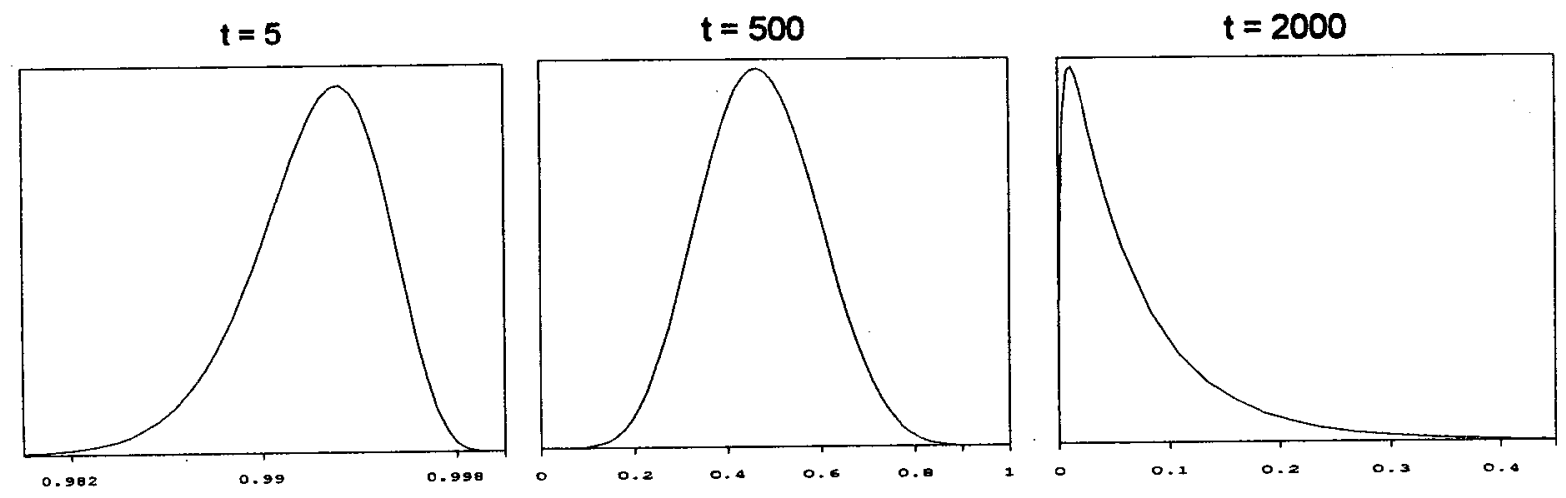

Figura 11: Densidades a posteriori $p_{1}(r /$ dados $)$, para $t_{0}=5,500$ e 2000 horas, considerando uma densidade a priori não-informativa de Jeffreys. 

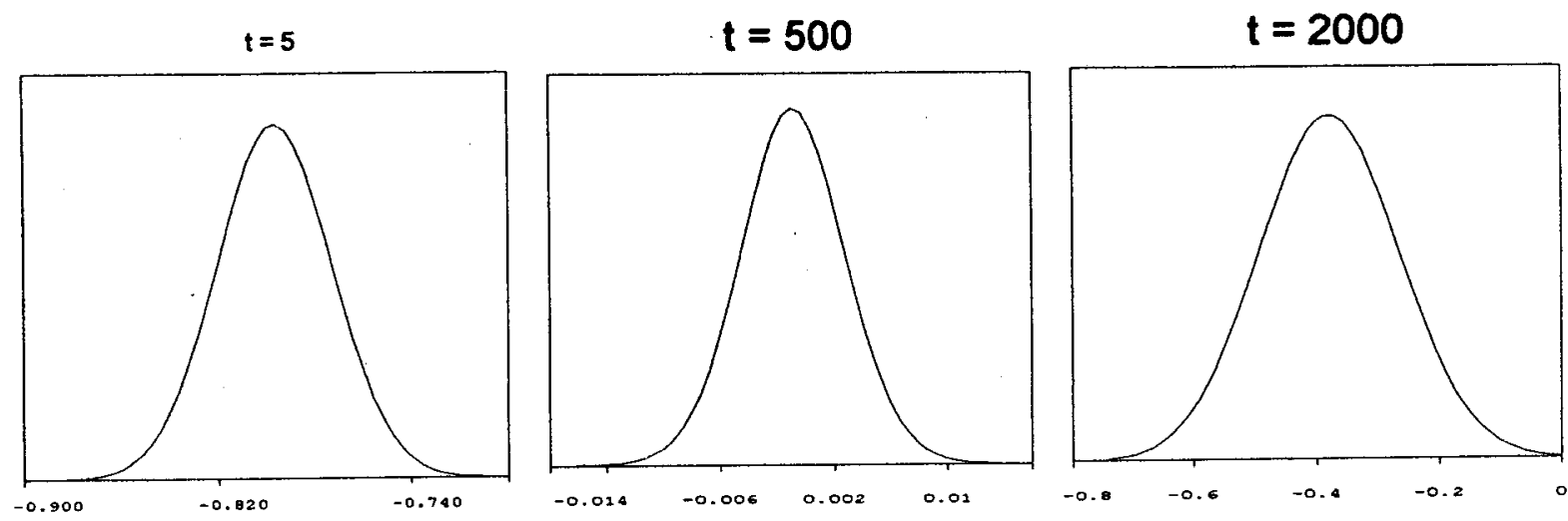

Figura 12: Densidades a posteriori $p_{1}\left(\phi_{G J} /\right.$ dados $)$, para $t_{0}=5,500$ e 2000 horas, considerando uma densidade a priori não-informativa de Jeffreys.
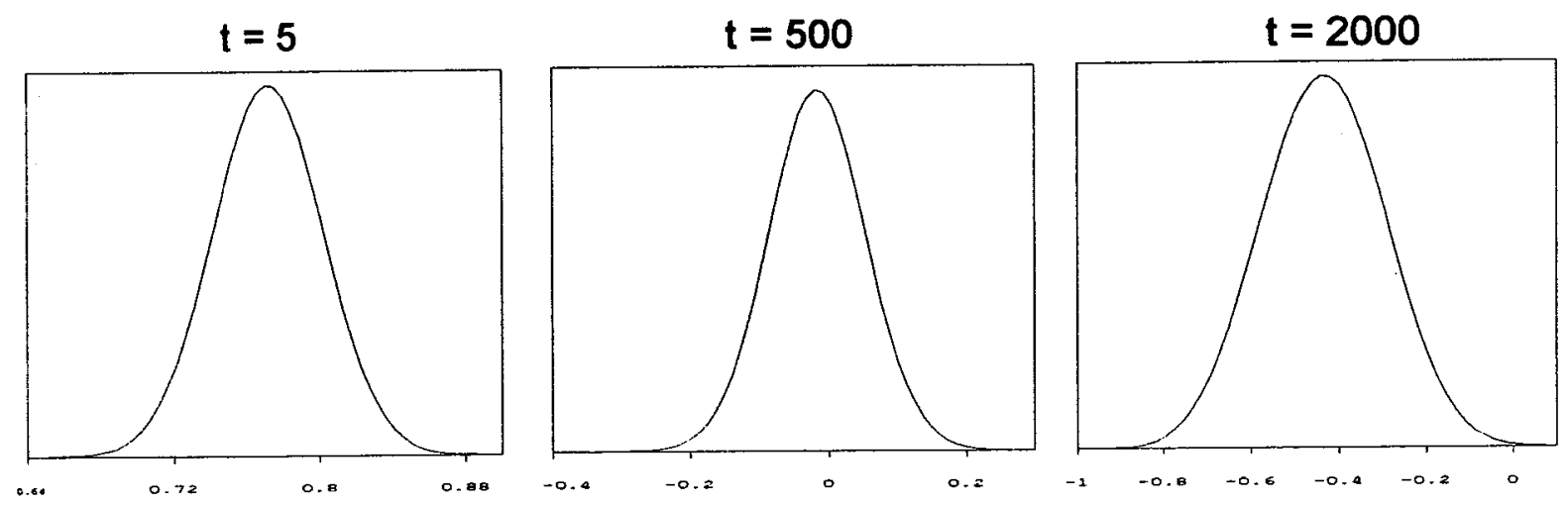

Figura 13: Densidades a posteriori $p_{1}\left(\phi_{A O} /\right.$ dados $)$, para $t_{0}=5,500$ e 2000 horas, considerando uma densidade a priori não-informativa de Jeffreys
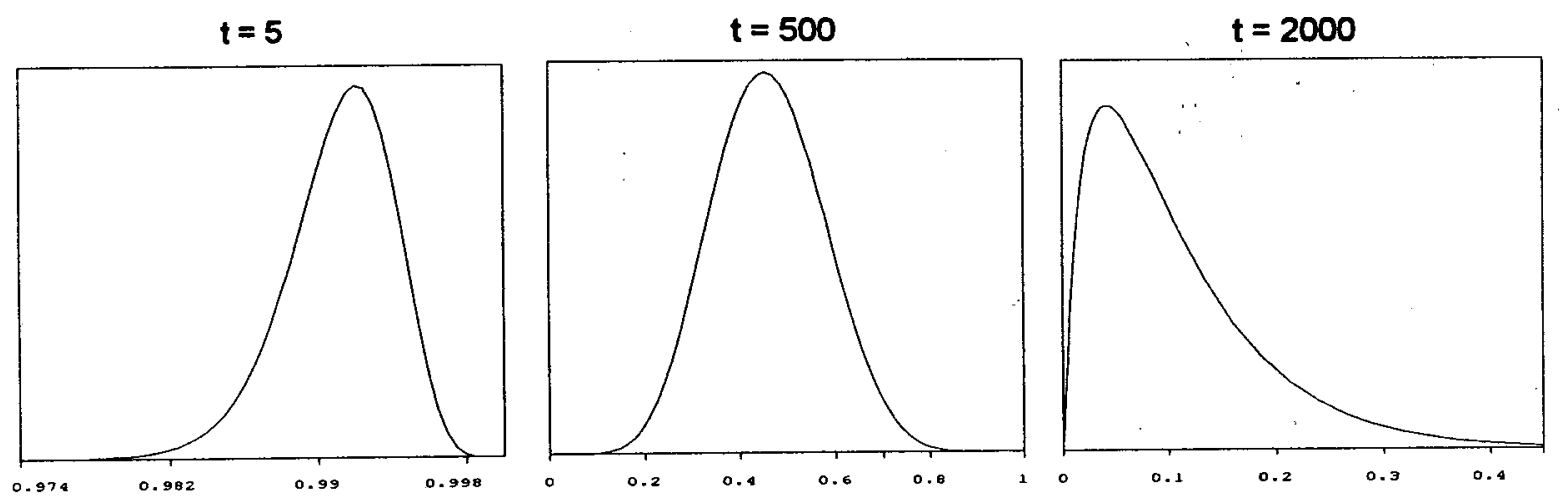

Figura 14: Densidades a posteriori $p_{2}(r /$ dados $)$, para $t_{0}=5,500$ e 2000 horas, considerando uma densidade a priori uniforme. 

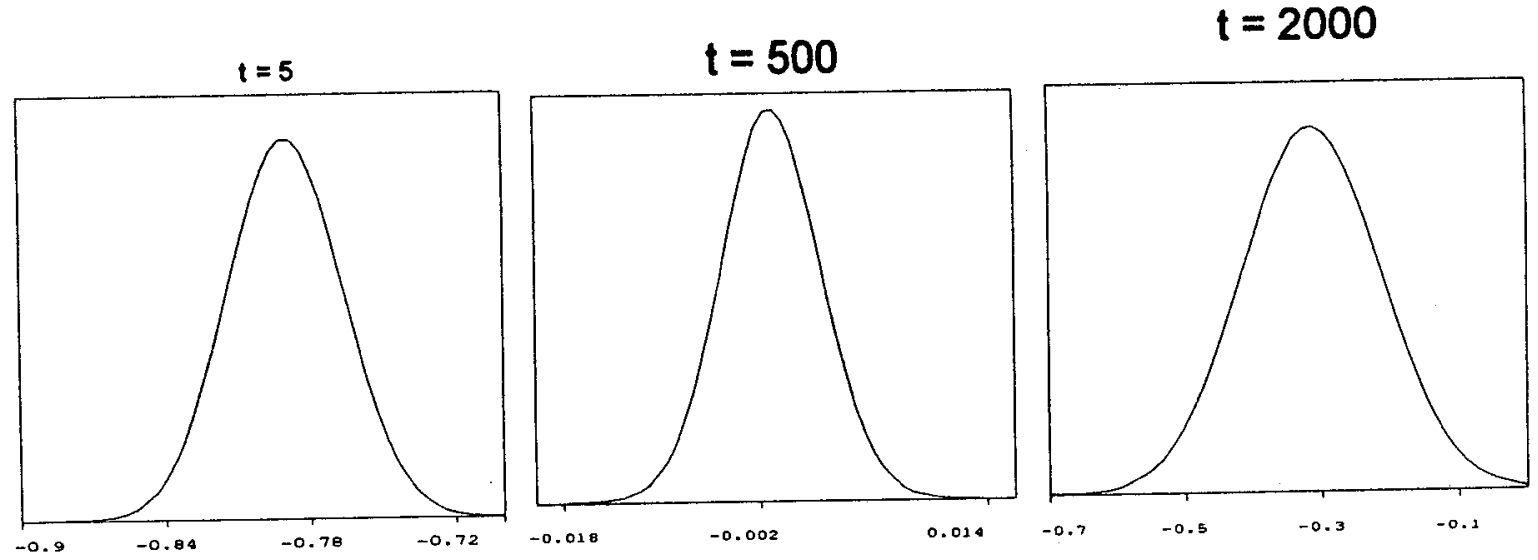

Figura 15: Densidades a posteriori $p_{2}\left(\phi_{G J} /\right.$ dados $)$, para $t_{0}=5,500$ e 2000 horas, considerando uma densidade a priori uniforme.
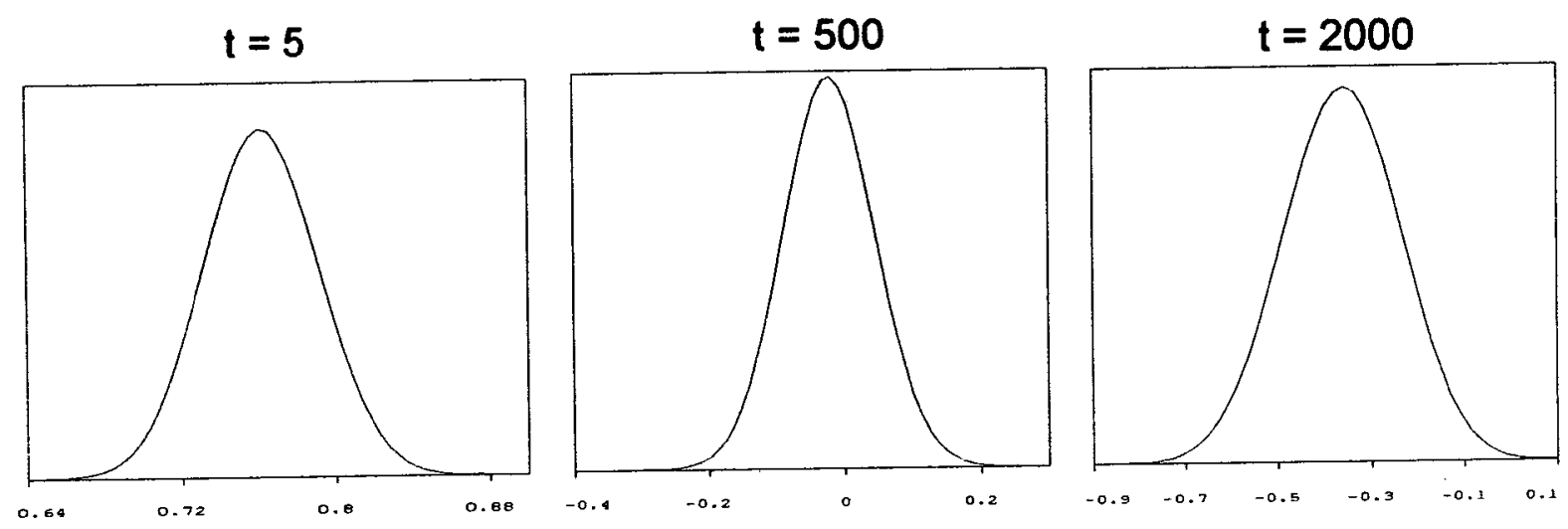

Figura 16: Densidades a posteriori $p_{2}\left(\phi_{A O} /\right.$ dados $)$, para $t_{0}=5,500$ e 2000 horas, considerando uma densidade a priori uniforme. 


\subsection{Exemplo para um Sistema com uma Unidade, Considerando o Modelo de Weibull.}

\section{Análise Clássica.}

Considere um conjunto de dados com censuras de tipo II introduzidos por Lawless (1982), p. 152 , consistindo de $n=40$ unidades, onde o experimento termina quando for observadas $d=28$ falhas (ver tabela 5). Assumindo o modelo de Weibull com densidade (5.1), os estimadores de máxima verossimilhança para os parâmetros $\alpha$ e $\beta$ obtidos pelo método de Newton-Raphson, são dados por:

$$
\left\{\begin{array}{l}
\hat{\alpha}=1.1692 \\
\hat{\beta}=1.0984
\end{array}\right.
$$

Desta forma, pela propriedade de invariância, o estimador de máxima verossimilhança para a função de confiabilidade num instante $t_{0}=5.0$ é dado por:

$$
\hat{R}(5.0)=\exp \left[-\left(\frac{5.0}{\hat{\alpha}}\right)^{\hat{\beta}}\right]=0.0072
$$

Considerando a reparametrização de Guerrero e Johnson para a confiabilidade, $\operatorname{com} \theta=\ln \beta$, temos que o estimador de máxima verossimilhança para o parâmetro $\theta$ é dado por $\hat{\theta}=\ln \hat{\beta}=0.0939$.

Já, para o parâmetro $\phi_{G J}(\lambda)$ obtivemos duas estimativas para dois valores distintos de $\lambda$. Os valores de $\lambda$ considerados foram aqueles que minimizaram 
respectivamente a terceira derivada padronizada do logaritmo da verosimilhança, $\operatorname{STD}\left[\hat{\phi}_{G J}(\lambda)\right]$, e a medida global de não normalidade de Kass e Slate, $m^{2} \bar{B}^{2}\left[\hat{\phi}_{G J}(\lambda), \hat{\theta}\right]$, apresentadas em (2.12) e (2.13), respectivamente.

No primeiro caso encontramos $\lambda=0.1269$, que implica num estimador de máxima verossimilhança para $\phi_{G J}(0.1269)$ dado por:

$$
\hat{\phi}_{G J}(0.1269)=-0.4650
$$

Por outro lado, o valor de $\lambda$ que minimiza $m^{2} \bar{B}^{2}\left[\hat{\phi}_{G J}(\lambda), \hat{\theta}\right]$ é dado por $\lambda=0.1820$, e o estimador de máxima verossimilhança para $\phi_{G J}(0.1820)$

$$
\hat{\phi}_{G J}(0.1820)=-0.5921 \text {. }
$$

\begin{tabular}{|llllll|}
\hline 0.0507 & 0.0579 & 0.0784 & 0.0954 & 0.1376 & 0.2249 \\
0.2362 & 0.2481 & 0.2501 & 0.2811 & 0.3027 & 0.3091 \\
0.4295 & 0.5379 & 0.5621 & 0.5781 & 0.7811 & 0.8228 \\
0.9455 & 0.9871 & 1.0060 & 1.0335 & 1.0377 & 1.0471 \\
0.0876 & 1.2473 & 1.2776 & 1.3445 & & \\
\hline
\end{tabular}

Tabela 5: Tempos de vida em horas. 
$\mathrm{Na}$ tabelas 6 e 7 temos, respectivamente, os valores de $\lambda$ obtido via a minimização da terceira derivada padronizada do logaritmo da verossimilhança e da medida global de não normalidade, para diversos valores de $t_{0}$, nas duas parametrizações.

Como podemos observar, há um ganho muito significativo na normalidade com a reparametrização de Guerrero e Johnson para a função de confiabilidade.

\begin{tabular}{|c|c|c|c|c|c|}
\hline$t_{0}$ & $\hat{R}\left(t_{0}\right)$ & $S T D\left[\hat{R}\left(t_{0}\right)\right]$ & $\lambda$ & $\hat{\phi}_{G J}(\lambda)$ & $S T D\left[\hat{\phi}_{G J}(\lambda)\right]$ \\
\hline \hline 0.01 & 0.9947 & 0.3749 & -0.3298 & -0.8216 & 0.0000 \\
0.40 & 0.7350 & 0.2034 & -0.1612 & -0.1517 & 0.0000 \\
1.00 & 0.4308 & 0.0995 & 0.0198 & -0.0055 & 0.0000 \\
2.50 & 0.0998 & 0.9284 & 0.1606 & -0.2975 & 0.0000 \\
5.00 & 0.0072 & 2.4192 & 0.1269 & -0.4650 & 0.0000 \\
\hline
\end{tabular}

Tabela 6: Valores de $\lambda$ e Terceiras Derivadas Padronizadas.

\begin{tabular}{|c|c|c|c|c|c|}
\hline$t_{0}$ & $\hat{R}\left(t_{0}\right)$ & $m^{2} \bar{B}^{2}\left[\hat{R}\left(t_{0}\right), \hat{\beta}\right]$ & $\lambda$ & $\hat{\phi}_{G J}(\lambda)$ & $m^{2} \bar{B}^{2}\left[\hat{\phi}_{G J}(\lambda), \hat{\theta}\right]$ \\
\hline \hline 0.01 & 0.99467 & 5.6040 & -0.1763 & -0.6022 & 0.2810 \\
0.40 & 0.7350 & 0.0726 & -0.1558 & -0.1470 & 0.1133 \\
1.00 & 0.4308 & 0.1183 & -0.0296 & 0.0083 & 0.0376 \\
2.50 & 0.0998 & 1.2977 & 0.2119 & -0.3725 & 0.0180 \\
5.00 & 0.0072 & 18.9370 & 0.1820 & -0.5921 & 0.0309 \\
\hline
\end{tabular}

Tabela 7: Valores de $\lambda$ e Medidas Globais de não Nomalidade. 
A seguir apresentaremos os contornos da função de verossimilhança conjunta para $R\left(t_{0}\right)$ e $\beta$, considerando $t_{0}=0.01,1.0$ e 5.0 , para os dois valores de $\lambda$ obtidos anteriormente. Podemos verificar que, minimizar a medida de normalidade marginal, dada por $\operatorname{STD}\left[\hat{\phi}_{G J}(\lambda)\right]$, já seria suficiente para melhorar a normalidade assintótica dos estimadores de máxima verossimilhança. Vemos ainda que, o valor de $\lambda$ que minimiza $m^{2} \bar{B}^{2}\left[\hat{\phi}_{G J}(\lambda), \hat{\theta}\right]$ acrescenta muito pouca contribuição à essa normalidade, como podemos observar pelas figuras 18 a 20 .
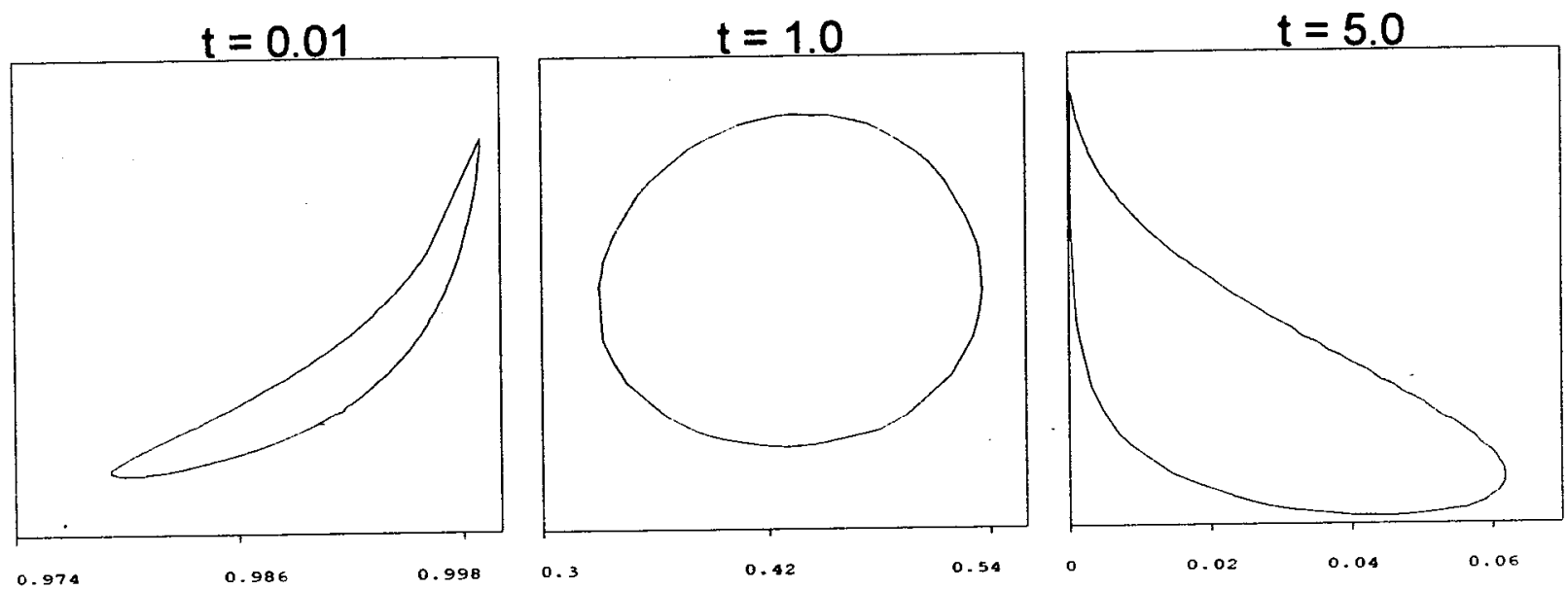

Figura 17: Contornos da verossimilhança conjunta na parametrização original $L(r, \beta)$.
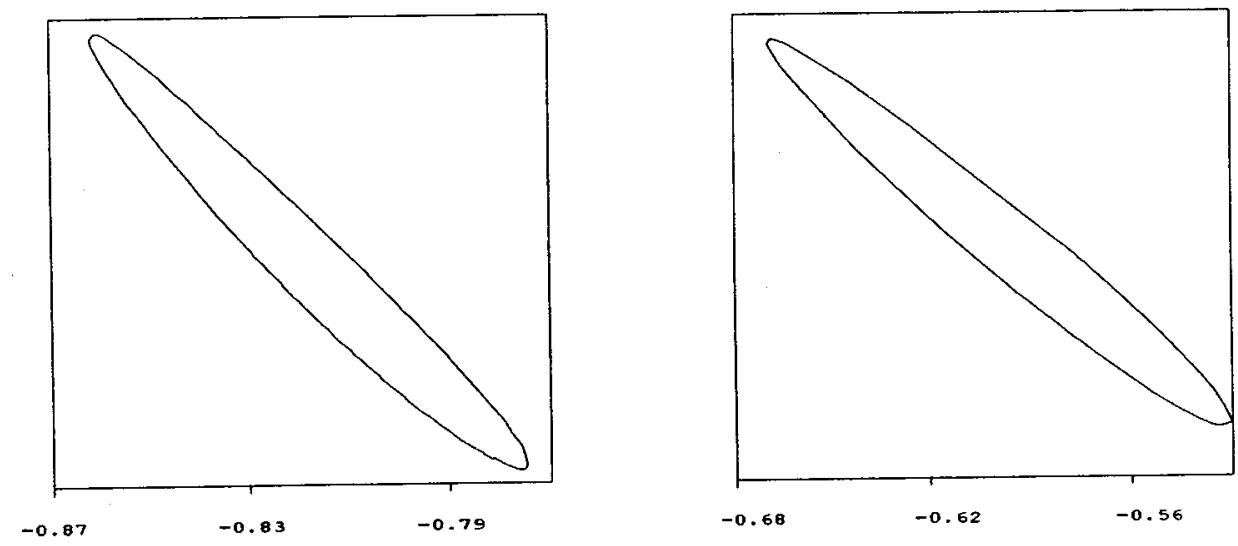

Figura 18: Contornos da verossimilhança conjunta $L\left(\phi_{G J}, \theta\right)$, para $t_{0}=0.01$, com $\lambda$ minimizando a $S T D$ e $m^{2} \bar{B}^{2}$, respectivamente. 

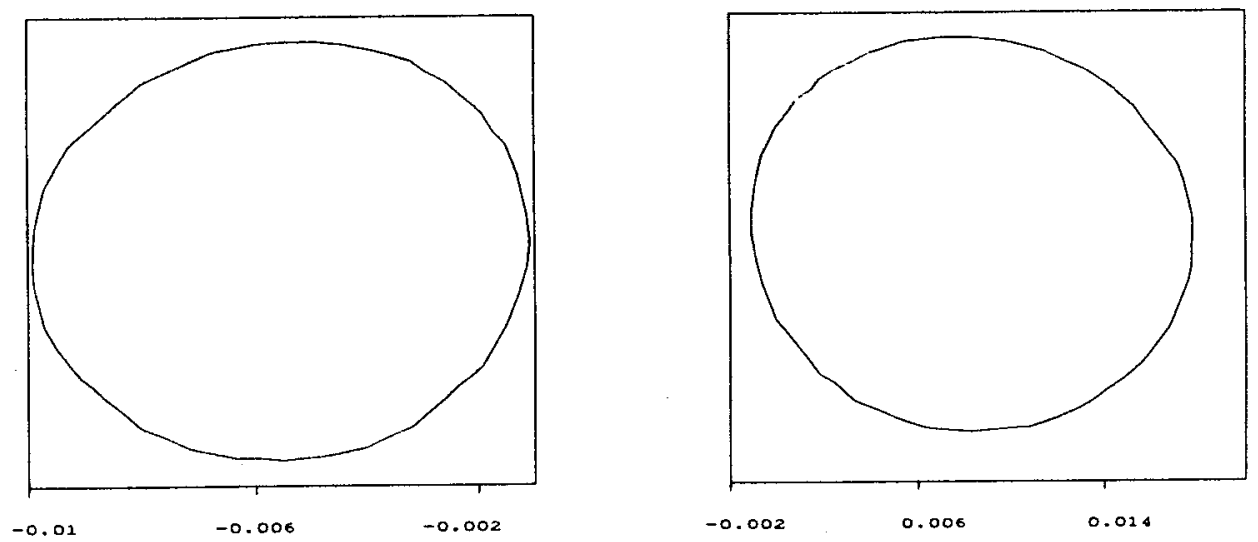

Figura 19: Contomos da verossimilhança conjunta $L\left(\phi_{G J}, \theta\right)$, para $t_{0}=1.0$, com $\lambda$ minimizando a $S T D$ e $m^{2} \bar{B}^{2}$, respectivamente.
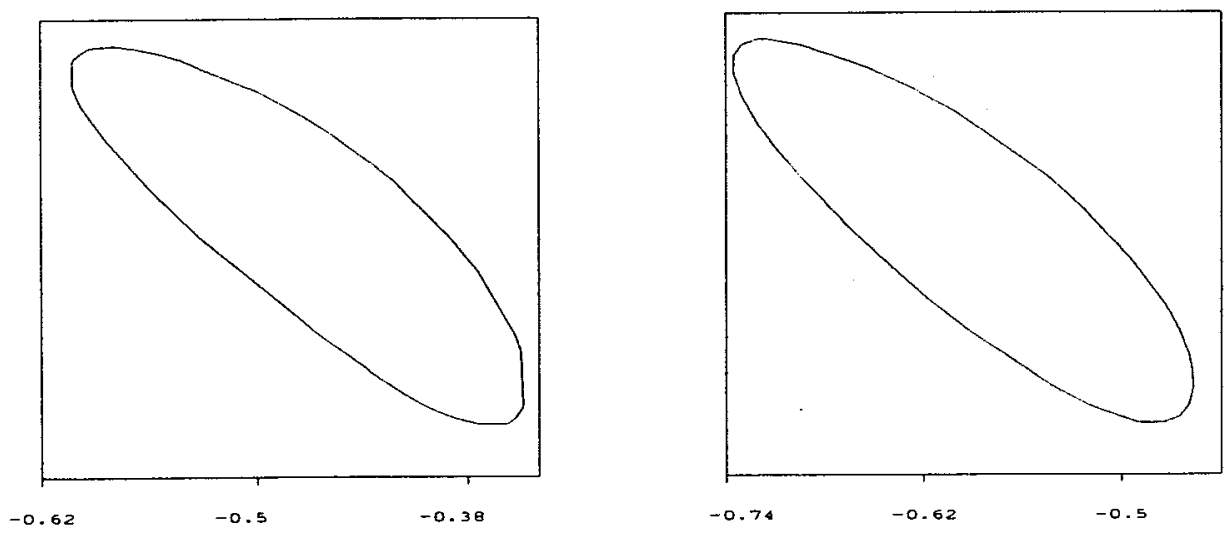

Figura 20: Contornos da verossimilhança conjunta $L\left(\phi_{G J}, \theta\right)$, para $t_{0}=5.0$, com $\lambda$ minimizando a $S T D$ e $m^{2} \bar{B}^{2}$, respectivamente.

Apresentamos nas figuras 21 a 24 os gráficos da verossimilhança "profile" e seus respectivos T-plot's, assumindo $t_{0}=0.01,1.0$ e 5.0, na parametrização original e na reparametrização de Guerrero e Johnson, com $\lambda$ obtido via STD. Observamos que os gráficos T-plot's na parametrização original, $T(r)$, são marcadamente curvos, indicando a não normalidade da função de verossimilhança "profile" $L_{\hat{\beta}}(r)$ enquanto que na 
reparametrização de Guerrero e Johnson os gráficos apresentam uma boa linearidade, indicando a normalidade da verosimilhança "profile" $L_{\hat{\theta}}\left(\phi_{G J}\right)$.

Com $\beta=1.0984$ conhecido, na tabela 8 temos intervalos de confiança $95 \%$ para a função de confiabilidade considerando a normalidade assintótica do estimadores de máxima verossimilhança, comparados com os intervalos obtidos através da distribuição: $2 T / \alpha^{\beta} \sim \chi_{2 d}^{2}$, onde $T=\sum_{i=1}^{d} t_{(i)}^{\beta}+(n-d) t_{(d)}^{\beta}$. Como podemos notar, houve uma sensível melhoria nos resultados obtidos pela a reparametrização de Guerrero e Johnson com o valor apropriado de $\lambda$, como podemos ver pelos valores da tabela, especialmente aqueles referentes aos instantes $t_{0}=2.50,3.0$ e 5.0 .

\begin{tabular}{|c|c|c|c|c|}
\hline$t_{0}$ & $\begin{array}{c}\text { Usando a } \\
\text { distribuição } \chi_{(2 d)}^{2} \\
\text { para } 2 T / \alpha^{\beta}\end{array}$ & $\begin{array}{l}\text { Normalidade } \\
\text { assintótica } \\
\text { para } \hat{R}\left(t_{0}\right)\end{array}$ & $\begin{array}{c}\lambda \text { que } \\
\text { minimiza a } \\
S T D \\
\end{array}$ & $\begin{array}{c}\text { Normalidade } \\
\text { assintótica } \\
\text { para } \hat{\phi}_{G J}(\lambda)\end{array}$ \\
\hline 0.01 & $(0.9925 ; 0.9964)$ & $(0.9927 ; 0.9966)$ & -0.3298 & $(0.9824 ; 0.9964)$ \\
\hline 0.40 & $(0.6498 ; 0.8151)$ & $(0.6512 ; 0.8188)$ & -0.1612 & $(0.6466 ; 0.8125)$ \\
\hline 1.00 & $(0.3063 ; 0.5717)$ & $(0.2964 ; 0.5651)$ & 0.0198 & $(0.3037 ; 0.5662)$ \\
\hline 2.50 & $(0.0393 ; 0.2166)$ & $(0.0146 ; 0.1850)$ & 0.1606 & $(0.0381 ; 0.2114)$ \\
\hline 3.00 & $(0.0192 ; 0.1543)$ & $(-0.0026 ; 0.1224)$ & 0.1627 & $(0.0184 ; 0.1501)$ \\
\hline 5.00 & $(0.0009 ; 0.0378)$ & $(-0.0059 ; 0.0204)$ & 0.1269 & $(0.0009 ; 0.0365)$ \\
\hline
\end{tabular}

Tabela 8: Intervalos de Confiança $95 \%$ para $R\left(t_{0}\right)$. 

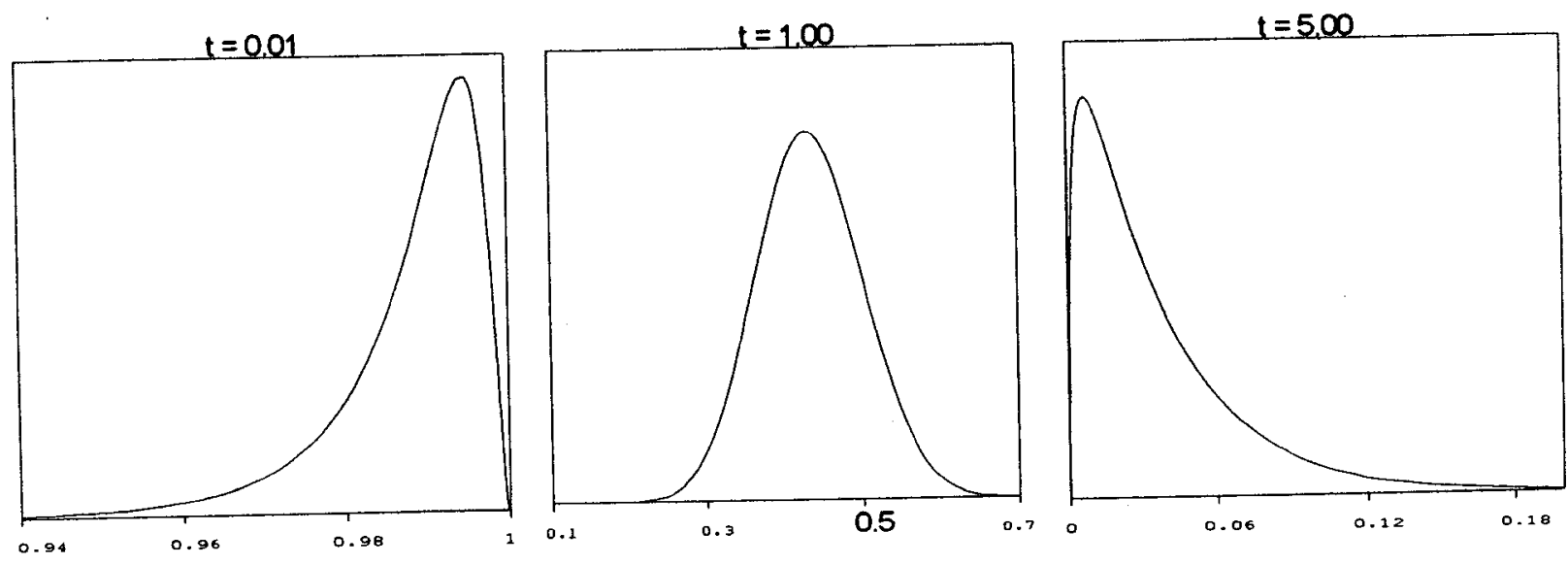

Figura 21: Verosimilhanças "Profile" $L_{\hat{\beta}}(r)$, para $t_{0}=0.01,1.0$ e 5.0 .
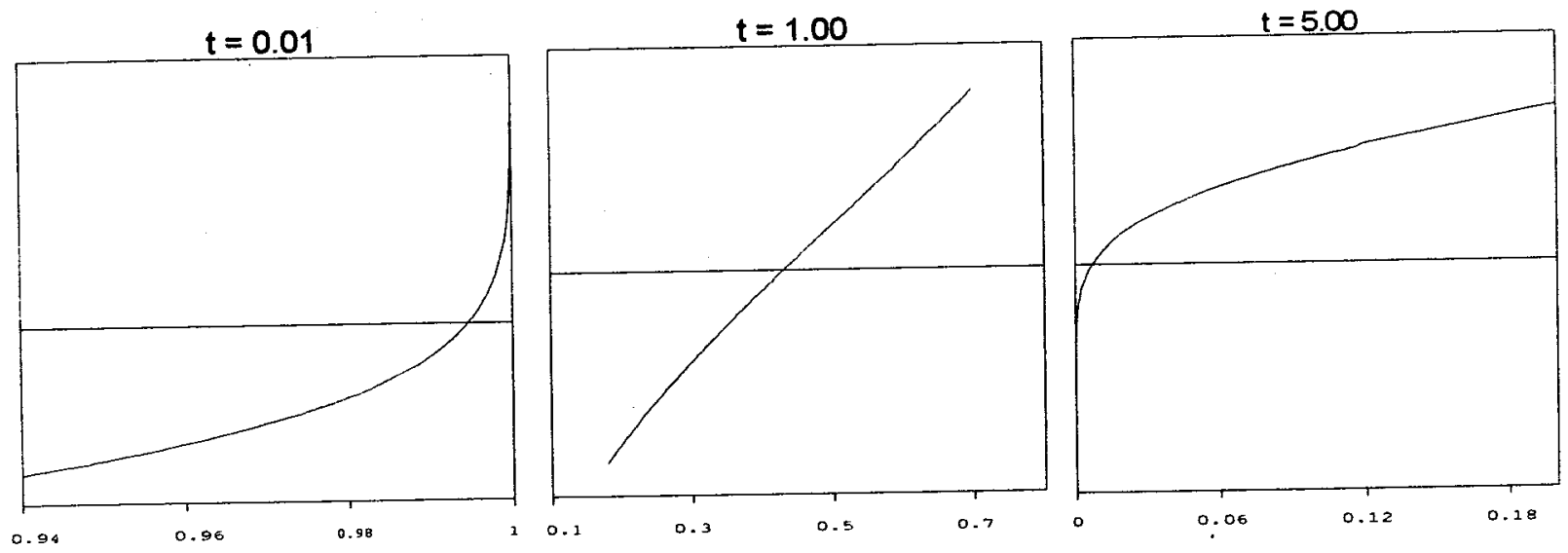

Figura 22: T-plot's das verosimilhanças "profile" $T_{\hat{\beta}}(r)$, para $t_{0}=0.01,1.0$ e 5.0 .
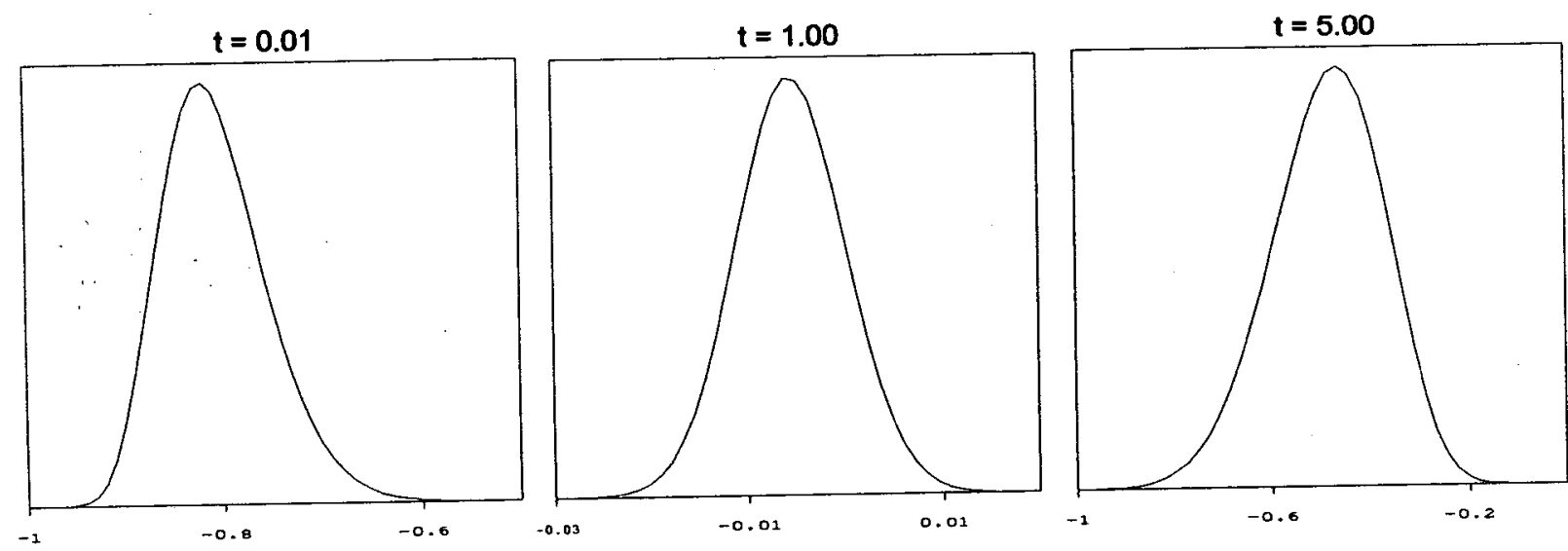

Figura 23: Verosimilhanças "Profile" $L_{\hat{\theta}}\left(\phi_{G J}\right)$, para $t_{0}=0.01,1.0$ e 5.0 . 

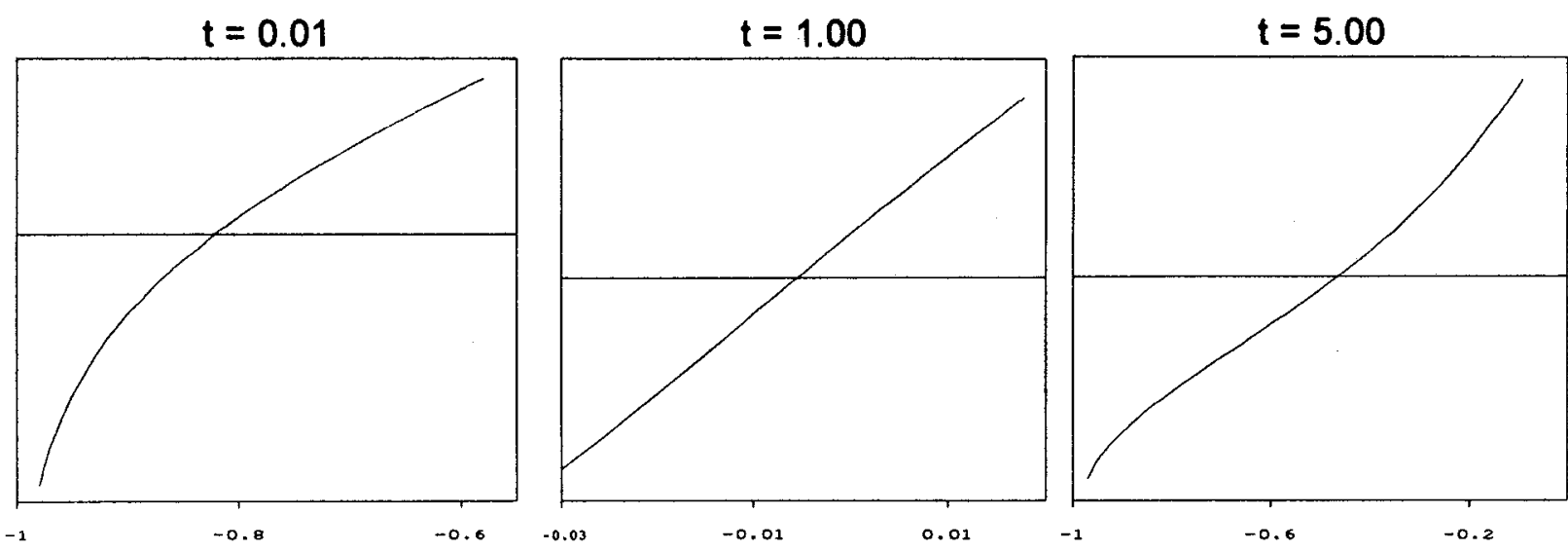

Figura 24: T-plot's das verosimilhanças "profile" $T_{\hat{\theta}}\left(\phi_{G J}\right)$, para $t_{0}=0.01,1.0$ e 5.0 .

\section{Análise Bayesiana.}

Considerando $\beta=1.0984$ conhecido e assumindo a densidade a priori dada em (5.45), a densidade a posteriori para $r=R\left(t_{0}\right)$, no instante $t_{0}=5.0$, é dada por:

$$
p(r / \text { dados }) \propto(-\ln r)^{27} r^{4.6753}, \quad 0 \leq r \leq 1
$$

Na parametrização de Guerrero e Johnson, com o valor de $\lambda$ que minimiza a STD, a densidade a posteriori para $\phi_{G J}=\phi_{G J}(\lambda)$, no instante $t_{0}=5.0$, é dada por:

$$
p\left(\phi_{G J} / \text { dados }\right) \propto B\left(\phi_{G J}\right)^{27}\left[1+A\left(\phi_{G J}\right)\right]^{-6.6753}\left(1+\phi_{G J}\right)^{-8.8770},-1 \leq \phi_{G J}<\infty .
$$

Nas figuras 25 e 26 temos os gráficos das densidades a posteriori (7.14) e (7.15), respectivamente. Novamente observamos a eficiência da reparametrização de Guerrero e Johnson em melhorar a normalidade.

A esperança a posteriori para $R\left(t_{0}\right) \operatorname{com} \beta$ conhecido é dada por: 


$$
E_{R}(R / \text { dados })=\left(1+\frac{t_{0}^{\beta}}{T}\right)^{-d}, \text { onde } T=\sum_{i=1}^{d} t_{(i)}^{\beta}+(n-d) t_{(d)}^{\beta} .
$$

Pata $t_{0}=5.0$, temos: $E_{R}(R /$ dados $)=0.0106$

Na parametrização de Guerrero e Johnson, a esperança a posteriori para $R\left(t_{0}\right)$ é dada por:

$$
E_{\phi_{G J}}(R / \text { dados })=E_{\phi_{G J}}\left\{\left[1+\left(1+\phi_{G J}\right)^{-1 / \lambda}\right]^{-1} / \text { dados }\right\}
$$

sendo obtida pelo método de Laplace para aproximação de integrais.

Na tabela 9, temos as esperanças a posteriori para a confiabilidade obtidas pelo método de Laplace comparadas com o valor dado em (7.16), onde podemos verificar a influência da parametrização na precisão dos resultados. Como podemos notar os erros relativos são bem menores para as estimativas considerando a reparametrização de Guerrero e Johnson.

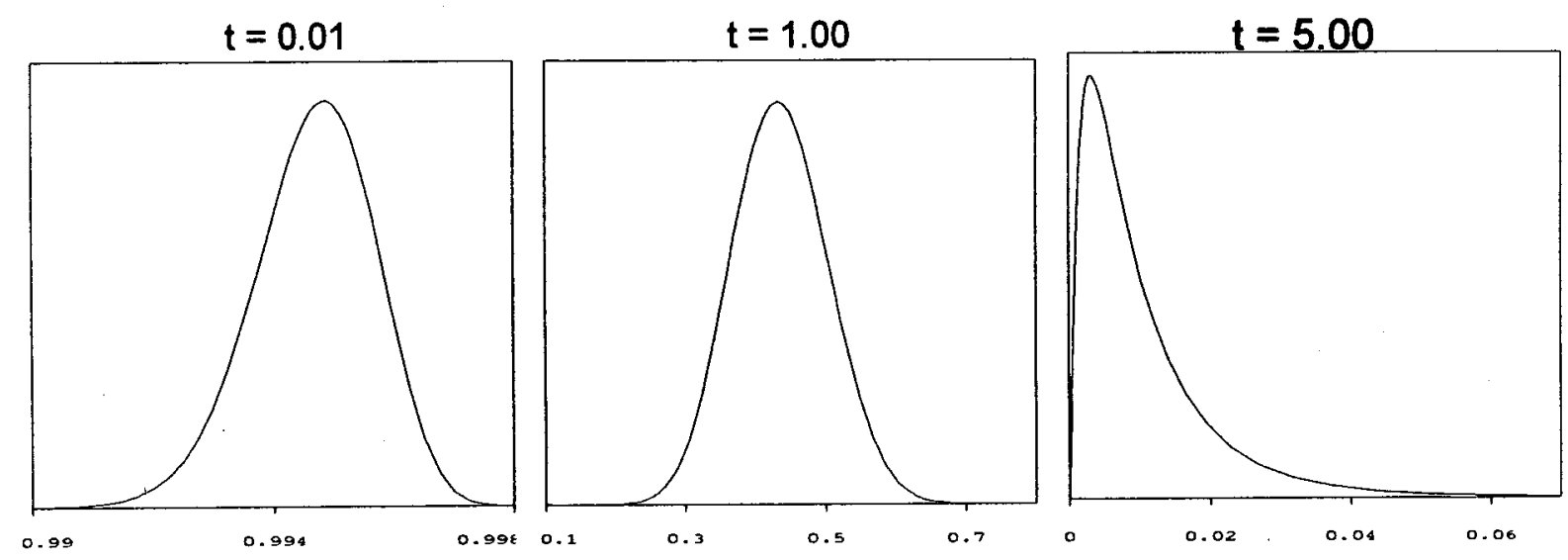

Figura 25: Densidades a posteriori na parametrização original. 

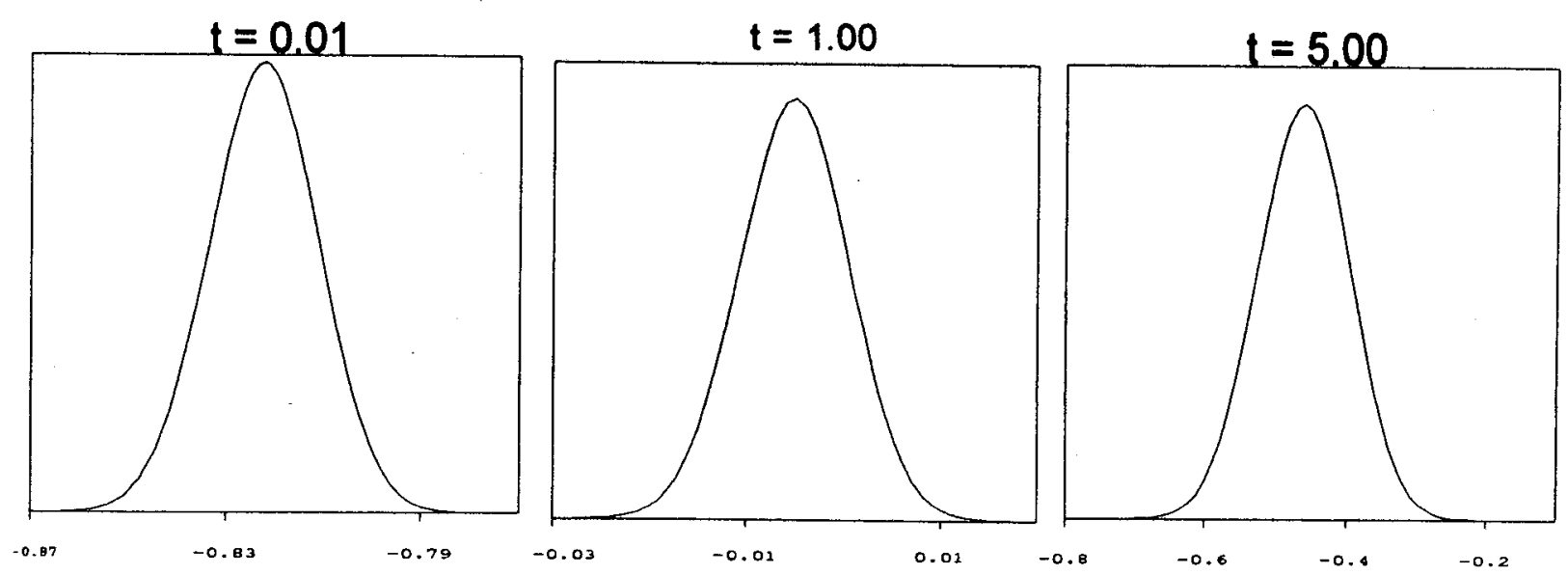

Figura 26: Densidades a posteriori na parametrização Guerrero e Johnson.

\begin{tabular}{|c|c|cc|cc|}
\hline \multirow{2}{*}{$t_{0}$} & \multicolumn{4}{|c|}{$E\left[R\left(t_{0}\right) /\right.$ dados $]$ aproximada pelo método de Laplace } \\
\cline { 2 - 6 } & Dada por & & Erro rel. & & Erro rel. \\
& $(7.16)$ & $R\left(t_{0}\right)$ & $\%$ & $\phi_{G J}(\lambda)$ & $\%$ \\
\hline 0.01 & 0.9947 & 0.9947 & $(0.000)$ & 0.9947 & $(0.000)$ \\
0.40 & 0.7363 & 0.7362 & $(0.008)$ & 0.7363 & $(0.002)$ \\
1.00 & 0.4361 & 0.4361 & $(0.013)$ & 0.4362 & $(0.004)$ \\
2.50 & 0.1092 & 0.1102 & $(0.902)$ & 0.1092 & $(0.072)$ \\
3.00 & 0.0684 & 0.0697 & $(1.914)$ & 0.0683 & $(0.122)$ \\
5.00 & 0.0106 & 0.0122 & $(14.394)$ & 0.0106 & $(0.283)$ \\
\hline
\end{tabular}

Tabela 9: Esperanças a Posteriori nas parametrizações $R\left(t_{0}\right), \phi_{G J}(\lambda)$, considerando uma densidade a priori dada em (5.45), comparadas com com o valor exato (erros relativos em \%). 


\subsection{Exemplo com Testes Acelerados Considerando o Modelo de Lei de Potência Inversa.}

\section{Análise Clássica.}

Para o exemplo com testes acelerados consideramos os dados gerados por Achcar e Dias (1990) segundo uma distribuição exponencial para os tempos de vida e o modelo de lei de potência inversa com $\gamma=0.8$ e $\delta=500$ (ver tabela 10). Os dados foram gerados em $k=5$ níveis de stress, num esquema de censuras tipo II onde apenas os primeiros $d_{i}, i=1,2, \ldots, 5$, tempos de vida são observados. Portanto, o número total de falhas observadas foi $d=\sum_{i=1}^{5} d_{i}=65$.

\begin{tabular}{|c|c|c|c|l|}
\hline$i$ & $V_{i}$ & $n_{i}$ & $d_{i}$ & $t_{i j}$ \\
\hline \hline 1 & 10 & 30 & 5 & $6,8,10,12,14$ \\
\hline 2 & 20 & 30 & 8 & $4,5,5,6,8,8,9,14$ \\
\hline 3 & 30 & 30 & 12 & $2,3,3,5,6,7,7,8,8,9,10,17$ \\
\hline 4 & 40 & 30 & 18 & $3,3,4,5,6,6,8,9,10,10,12,12,13,14,14,15,24$ \\
\hline 5 & 50 & 30 & 22 & $\begin{array}{l}2,3,4,5,5,8,8,8,9,10,12,13,14,14,15,18,18, \\
18,19,20,20,27\end{array}$ \\
\hline
\end{tabular}

Tabela 10: Dados de tempos de vida gerados por Achcar e Dias (1990).

Assumindo o modelo exponencial para os tempos de vida, os estimadores de máxima verossimilhança para $\gamma$ e $\delta$ são dados por: 


$$
\left\{\begin{array}{l}
\hat{\delta}=500.5555 \\
\hat{\gamma}=0.7995
\end{array}\right.
$$

Testes acelerados são normalmente utilizados para se obter inferências aos níveis usuais de stress (condições normais de uso), quando o tempo médio de vida de um produto é muito alto. Desta forma, considerando o nível usual de stress, ou seja, com $V_{1}=10$, os estimadores de máxima verossimilhança para o tempo médio de vida e para a confiabilidade num dado instante $t_{0}=200$, são dados por:

$$
\left\{\begin{array}{l}
\hat{\alpha}_{1}=79.4332 \\
\hat{r}_{1}=\exp \left(-\frac{200 V_{i}^{\hat{\gamma}}}{\hat{\delta}}\right)=0.0806
\end{array}\right.
$$

Podemos melhorar a normalidade do estimador de máxima verossimilhança da confiabilidade através da transformação de Guerrero e Johnson com um valor apropriado de $\lambda_{1}$. Neste caso, vamos considerar o valor de $\lambda_{1}$ que minimiza a medida de não normalidade global de Kass e Slate (1992). Na tabela 11, temos os estimadores de máxima verossimilhança, nas duas parametrizações, e também os respectivos valores'de $m^{2} \bar{B}^{2}$, para $t_{0}=10,100,200$ e 400 .

Podemos notar que com a transformação de Guerrero e Johnson os valores das medidas estão mais próximos de zero, indicando que a normalidade assintótica dos estimadores de máxima verossimilhança é melhor nesta parametrização. Podemos também avaliar a normalidade dos estimadores de máxima verossimilhança pelos contornos das funções de verosimilhança conjuntas, dados nas figuras 27 e 28 . 


\begin{tabular}{|c|c|c|c|c|c|}
\hline$t_{0}$ & $\hat{R}_{1}\left(t_{0}\right)$ & $m^{2} \bar{B}^{2}\left(\hat{r}_{1}, \hat{\gamma}\right)$ & $\hat{\phi}_{G J_{1}}\left(\lambda_{1}\right)$ & $m^{2} \bar{B}^{2}\left(\hat{\phi}_{G J_{1}}, \hat{\gamma}\right)$ & $\lambda_{1}$ \\
\hline \hline 10 & 0.8817 & 0.8589 & 0.1918 & 0.0014 & 0.0874 \\
\hline 100 & 0.3201 & 0.4413 & -0.2563 & 0.0014 & 0.3201 \\
\hline 200 & 0.0806 & 5.9022 & -0.4739 & 0.0014 & 0.2639 \\
\hline 400 & 0.0065 & 35.5343 & -0.5263 & 0.0014 & 0.1486 \\
\hline
\end{tabular}

Tabela 11: Valores de $\lambda_{1}$ e medidas globais de não normalidade.
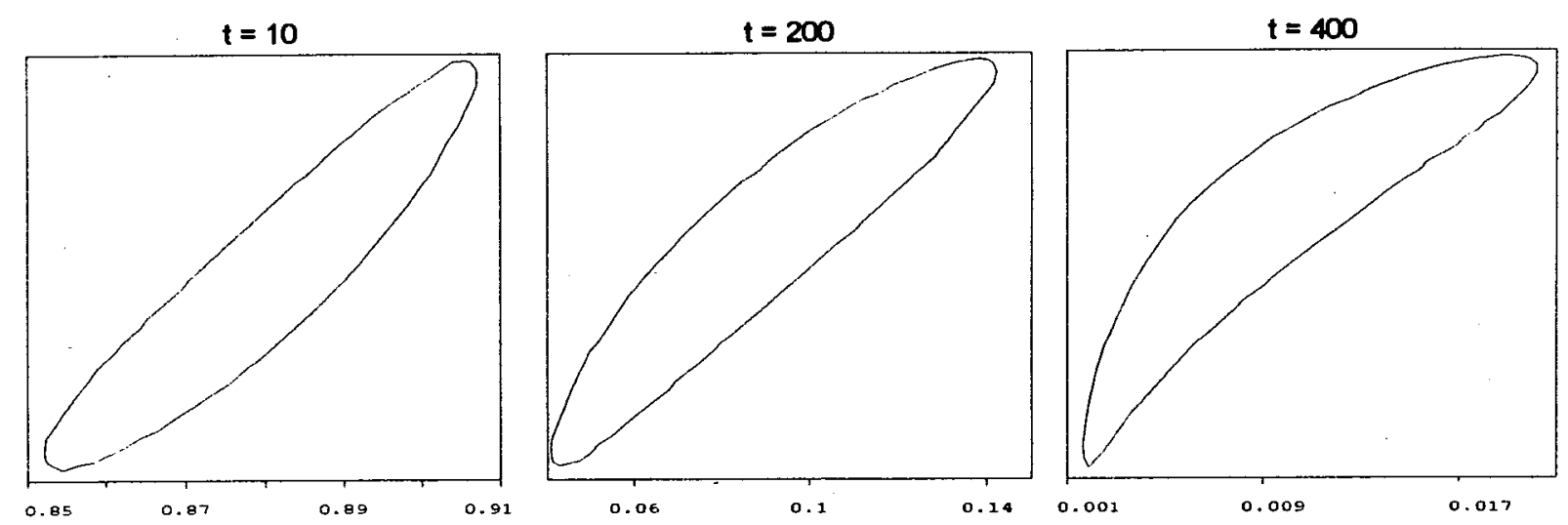

Figura 27: Contornos da verossimilhança conjunta $L\left(r_{1}, \gamma\right)$.
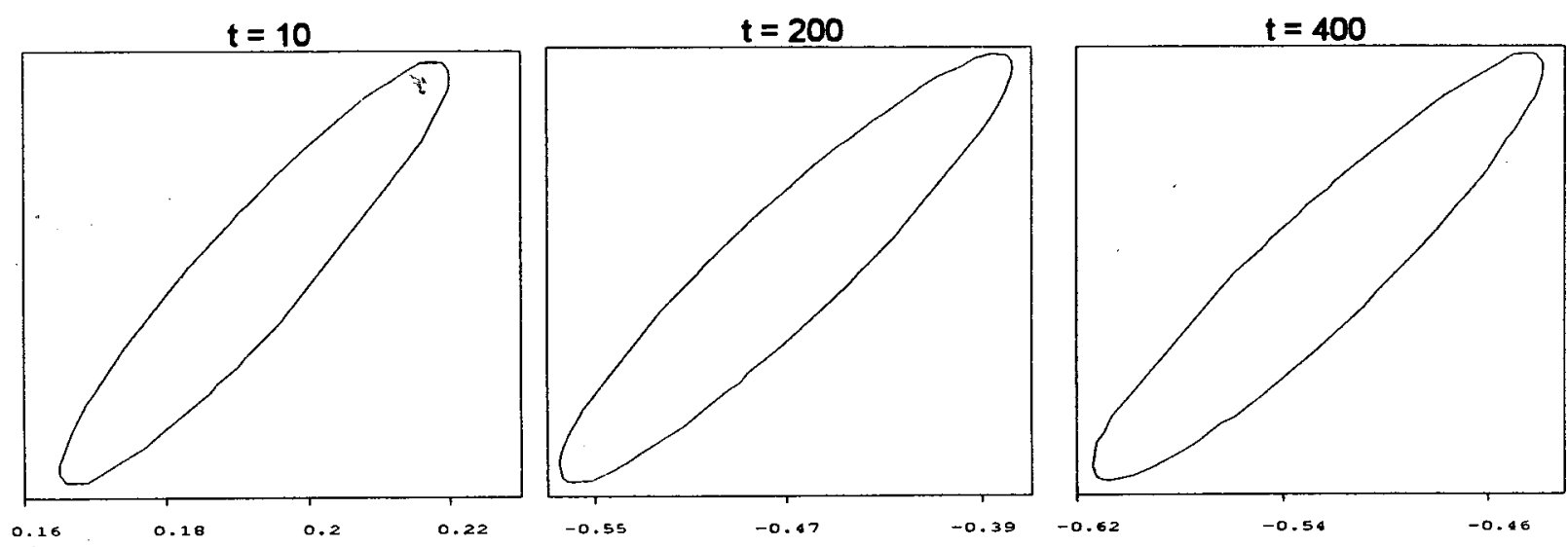

Figura 28: Contornos da verossimilhança conjunta $L\left(\phi_{G_{1}}, \gamma\right)$. 
Outra forma de se avaliar a normalidade de $R\left(t_{0}\right)$ é através da função de verossimilhança "profile" e do T-plot de Hills e Smith (1993), dados nas figuras 29 e 31 , respectivamente. Considerando a reparametrização de Guerrero e Jonhson melhoramos a normalidade da verossimilhança profile, melhora conforme podemos constatar através da figura 30 , onde temos a função de verossimilhança profile na nova parametrização, com $\lambda_{1}$ obtido via STD. Também verificamos a melhoria obtida na normalidade das verosimilhança "profiles" através da linearidade dos T-plot, na figura 32, comparados com os da parametrização original, na figura 31 .
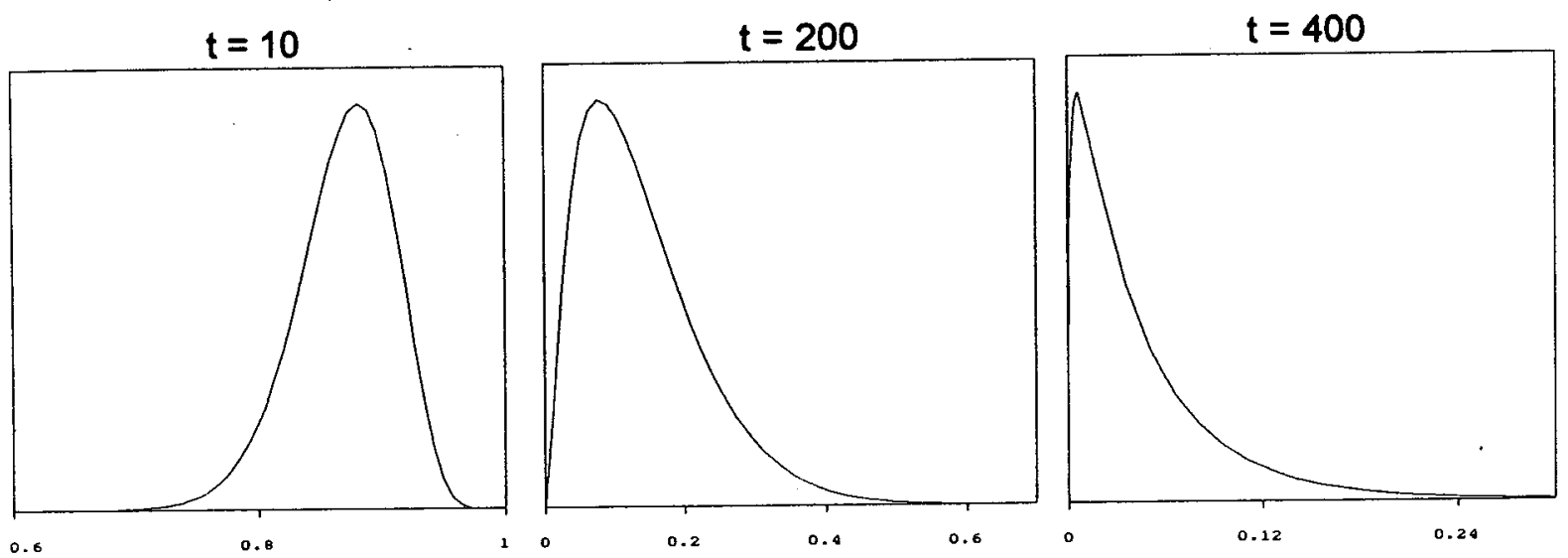

Figura 29: Verosimilhança "Profile" na parametrização original, $L_{\hat{\gamma}}\left(r_{1}\right)$.
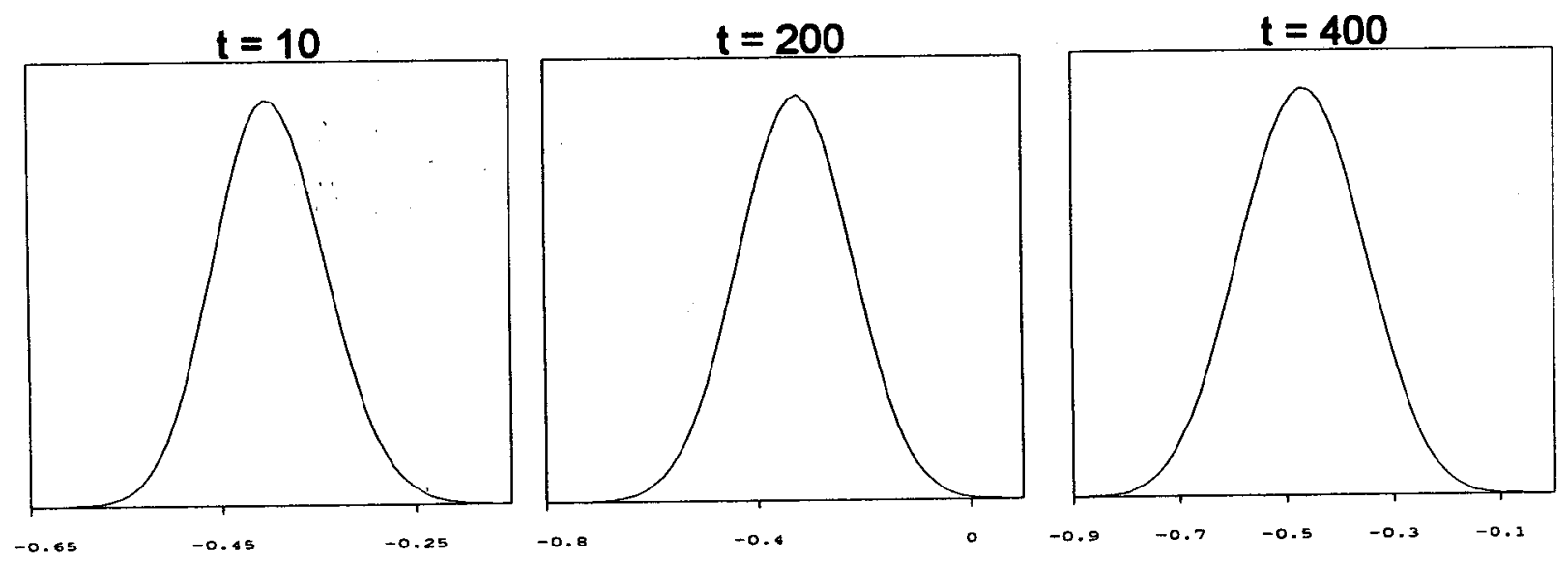

Figura 30: Verosimilhança "Profile" na parametrização $L_{\hat{\gamma}}\left[\phi_{G J_{1}}\left(\lambda_{1}\right)\right]$. 

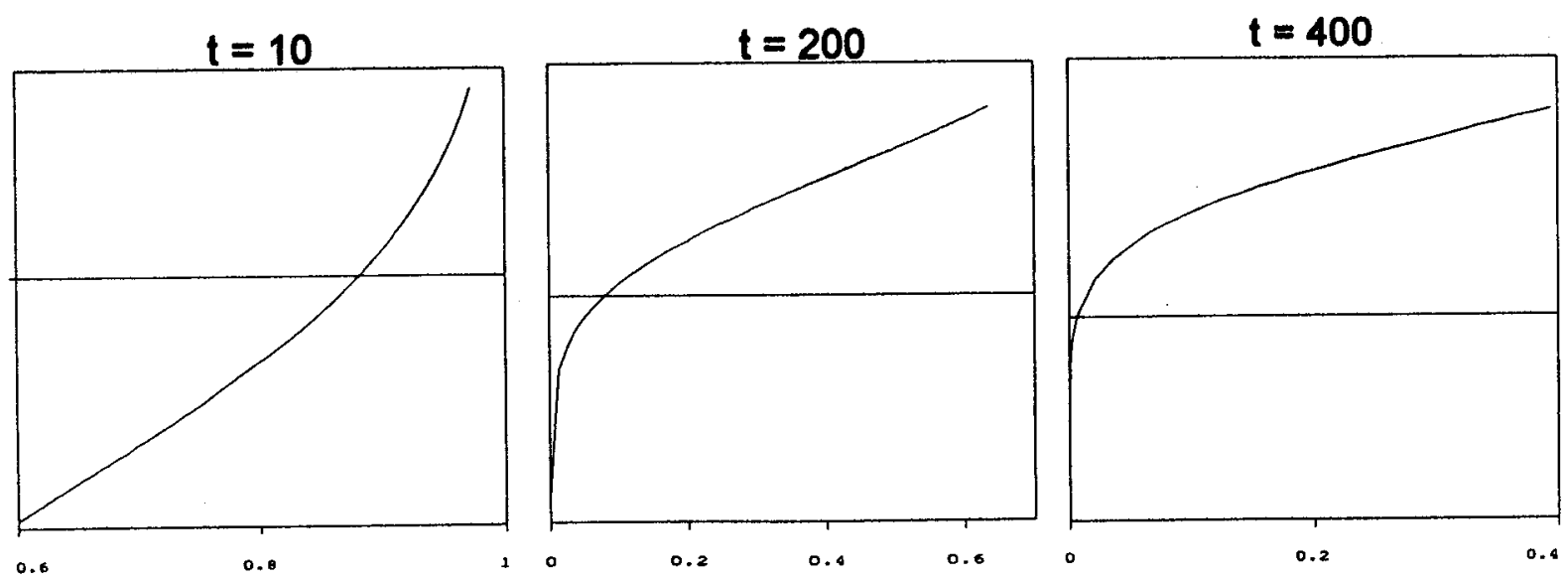

Figura 31: T-plot's das verosimilhança "Profile" na parametrização original, $T_{\hat{\gamma}}\left(r_{1}\right)$.
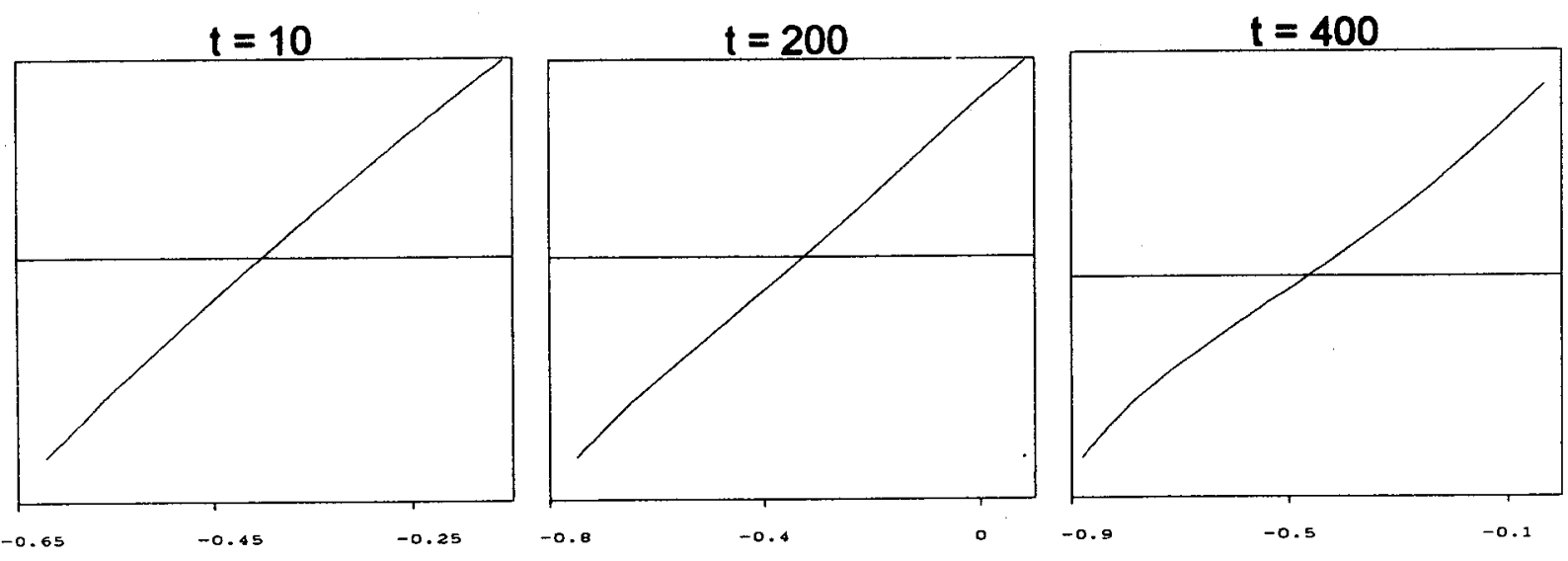

Figura 32: T-plot's das verosimilhança "Profile" na parametrização Guerrero e Johnson, $T_{\hat{\gamma}}\left[\phi_{G_{1}}\left(\lambda_{1}\right)\right]$.

Na tabela 12, assumindo $\gamma=0.7995$, temos os intervalos de confiança $95 \%$ para a confiabilidade considerando a normalidade assintótica do estimador de máxima verossimilhança nas parametrizações original e de Guerrero e Johnson, e também intervalos obtidos pela distribuição $2 T\left(-\ln r_{1}\right) / t_{0} \sim \chi_{(2 d)}^{2}$. Através dos resultados apresentados podemos observar que os intervalos assintóticos obtidos com a reparametrização de Guerrero e Johnson estão mais próximos daqueles obtidos pela distribuição exata $\chi_{(2 d)}^{2}$, especialmente para valores extremos de $t_{0}$ 


\begin{tabular}{|c|c|c|cc|}
\hline$t_{0}$ & distribuição $\chi_{(2 d)}^{2}$ & $\begin{array}{c}\text { Normalidade } \\
\text { assintótica } \\
\text { para } \hat{R}_{1}\left(t_{0}\right)\end{array}$ & $\begin{array}{c}\lambda \text { que } \\
\text { minimiza a }\end{array}$ & $\begin{array}{c}\text { Normalidade } \\
\text { assintótica } \\
\text { para } \hat{\phi}_{G J_{1}}(\lambda)\end{array}$ \\
\hline \hline 10 & $(0.8536,0.9074)$ & $(0.8547,0.9086)$ & -0.2553 & $(0.8530,0.9069)$ \\
100 & $(0.2054,0.3761)$ & $(0.1971,0.3709)$ & 0.0952 & $(0.2040,0.3761)$ \\
200 & $(0.0422,0.1433)$ & $(0.0313,0.1300)$ & 0.1628 & $(0.0415,0.1416)$ \\
400 & $(0.0018,0.0205)$ & $(-0.0015,0.0145)$ & 0.1250 & $(0.0017,0.0202)$ \\
\hline
\end{tabular}

Tabela 12: Intervalos de Confiança $95 \%$ para a Confiabilidade $R_{1}\left(t_{0}\right)$

\section{Análise Bayesiana assumindo $\gamma$ conhecido.}

Conforme a expressão (6.54), a densidade a posteriori para a confiabilidade assumindo uma densidade a priori de Jeffreys e $\gamma=0.7995$, com $t_{0}=200$ é dada por:

$$
p\left(r_{1} / \text { dados }\right) \propto\left(-\ln r_{1}\right)^{64} r_{1}^{24.8157}, \quad 0 \leq r_{1} \leq 1
$$

Considerando a reparametrização de Guerrero e Johnson, com $\lambda_{1}=0.1628$ obtido via $S T D$, a densidade a posteriori para $\phi_{G J_{1}}=\phi_{G_{J_{1}}}\left(\lambda_{1}\right)$ no instante $t_{0}=200$, obtida de (6.56), é dada por:

$$
p\left(\phi_{G J_{1}} / \text { dados }\right) \propto B\left(\phi_{G J_{1}}\right)^{64}\left[1+A\left(\phi_{G J_{1}}\right)\right]^{-26.8157}\left(1+\phi_{G J_{1}}\right)^{-7.1427}
$$

onde, $-1 \leq \phi_{G J_{1}}<\infty$. 
Nas figuras 33 e 34 temos os gráficos das densidades a posteriori (7.20) e (7.21), respectivamente, onde podemos avaliar os resultados obtidos com a reparametrização considerada.

O estimador Bayesiano para a confiabilidade segundo a função de perda quadrática é dado pela esperança a posteriori, que na parametrização original para $t_{0}=200$ é dada por:

$$
E_{R_{1}}\left(R_{1} / \text { dados }\right)=\left(1+\frac{200}{T}\right)^{-65}=0.0846
$$

onde $T=\sum_{i=1}^{k} T_{i} Q_{i}, T_{i}=\sum_{j=1}^{d_{i}} t_{i(j)}+\left(n_{i}-d_{i}\right) t_{i(d)}^{\beta}$ e $Q_{i}=\left(V_{i} / V_{p}\right)^{\gamma}$.

Como na parametrização de Guerrero e Johnson a esperança a posteriori para $R_{1}\left(t_{0}\right)$ não é determinada analiticamente, utilizamos do método de Laplace para obtêlas. Na tabela 13 podemos observar o efeito da reparametrização na precisão dos resultados com a utilização do método de Laplace. Como podemos ver, os erros relativos são menores na reparametrização de Guerrero e Johnson, especialmente para valores extremos de $t_{0}$.
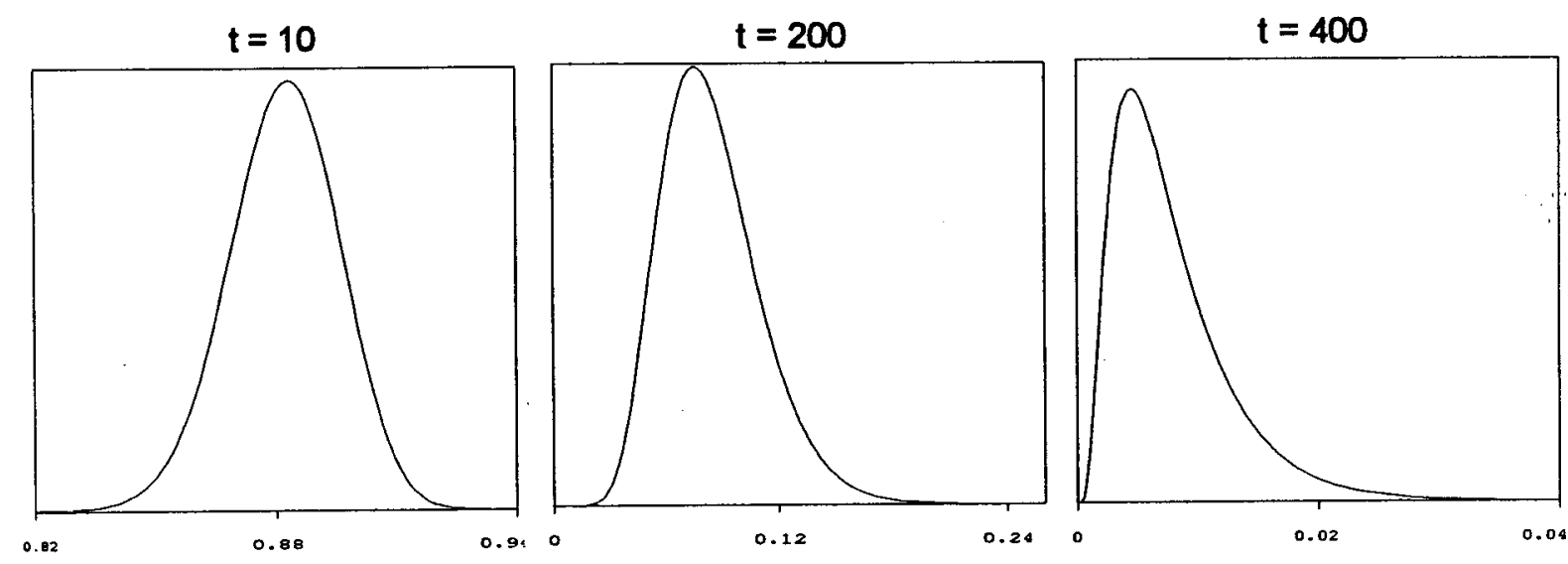

Figura 33: Densidades a posteriori na parametrização original. 

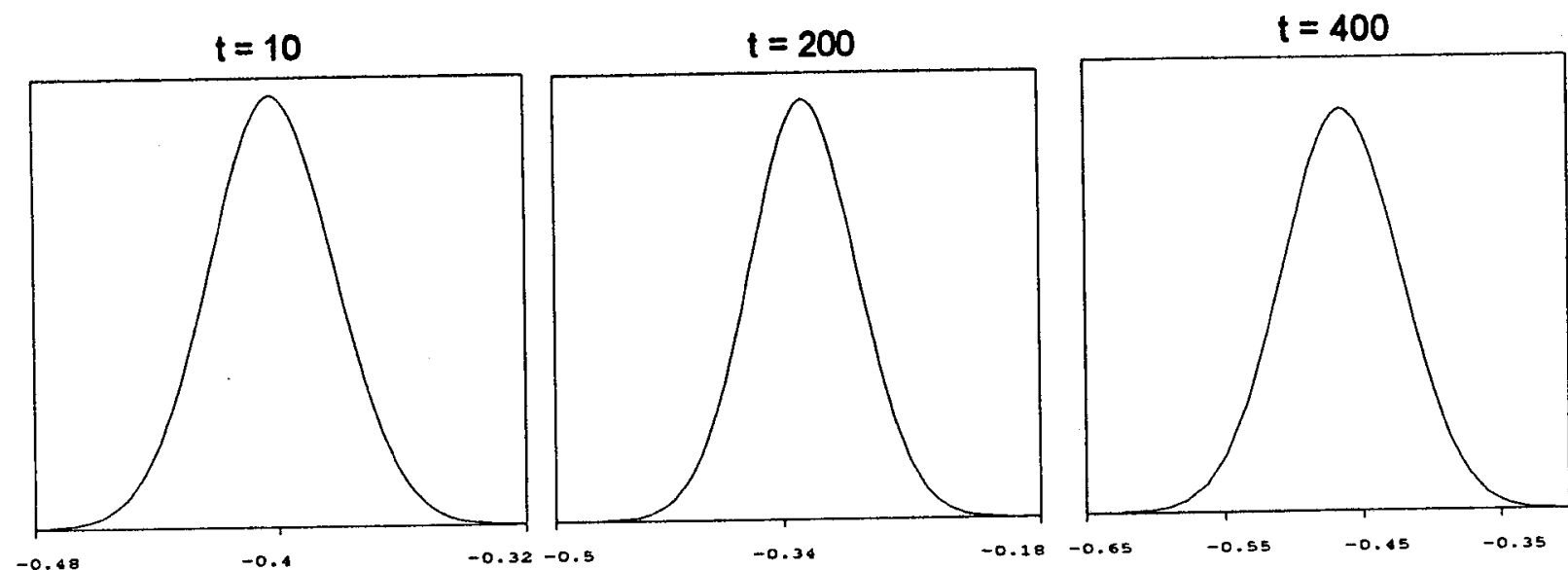

Figura 34: Densidades a posteriori na parametrização Guerrero e Johnson.

\begin{tabular}{|c|c|cc|cc|}
\hline \multirow{2}{*}{$t_{0}$} & \multicolumn{5}{|c|}{$E\left[R\left(t_{0}\right) /\right.$ dados $]$ aproximada pelo método de Laplace } \\
\cline { 2 - 6 } & DADA POR & & ERRO REL. & & ERRO REL. \\
& $(7.22)$ & $R_{1}\left(t_{0}\right)$ & $\%$ & $\phi_{G J_{1}}\left(\lambda_{1}\right)$ & $\%$ \\
\hline \hline 10 & 0.8818 & 0.8818 & $(0.000)$ & 0.8818 & $(0.000)$ \\
100 & 0.2874 & 0.2874 & $(0.009)$ & 0.2874 & $(0.002)$ \\
200 & 0.0846 & 0.0848 & $(0.203)$ & 0.0845 & $(0.018)$ \\
400 & 0.0078 & 0.0080 & $(2.540)$ & 0.0078 & $(0.052)$ \\
\hline
\end{tabular}

Tabela 9: Esperanças a Posteriori nas parametrizações $R_{1}\left(t_{0}\right), \phi_{G J_{1}}\left(\lambda_{1}\right)$, considerando uma densidade a priori de Jeffreys (erros relativos em \%). 


\section{CONSIDERAÇÕES FINAIS E PROPOSTAS FUTURAS.}

Nesta Dissertação de Mestrado avaliamos a utilização das reparametrizações de Guerrero e Johnson (2.3) e Aranda-Ordaz (2.5) para a confiabilidade de sistemas $R\left(t_{0}\right)$, visando melhorar a normalidade da função de verosimilhança e densidades a posteriori. Inicialmente consideramos os modelo exponencial e de Weibull para os tempos de vida de um sistema com uma unidade, utilizando amostras com dados censurados. Consideramos, também, uma aplicação da transformação de Guerrero e Johnson em testes acelerados com o modelo de lei de potência inversa.

Com as parametrizações consideradas, exploramos as medidas de normalidade introduzidas por Kass e Slate (1992), dada pela terceira derivada padronizada do logaritmo da função de verossimilhança $S T D$, ver (2.12), e pela medida global de nãonormalidade $m^{2} \bar{B}^{2}$, ver (2.13), para determinar o valor apropriado de $\lambda$ nas transformações, de forma a assegurar a normalidade assintótica dos parâmetros de interesse. Para checar a normalidade da função de verossimilhança, utilizamos o gráfico de T-Plot de Hills e Smith (1993).

No enfoque Bayesiano, tivèmos uma aproximação normal muito melhor para as densidades a posteriori, melhorando a precisão das inferências, principalmente quando utilizamos o método de Laplace para aproximação de integrais.

As técnicas desenvolvidas foram aplicadas em três exemplos práticos, onde verificamos que as transformações consideradas, com o valor de $\lambda$ apropriado, melhoraram significativamente a precisão das inferências, especialmente para amostras 
moderadas e valores extremos de $t_{0}$, como foi o caso do modelo exponencial (ver capítulo 7).

Com os resultados obtidos podemos concluir seguramente que, com as reparametrizações consideradas, aliadas às medidas de normalidade utilizadas na obtenção de $\lambda$, conseguimos uma melhoria considerável na aproximação normal das funções de verossimilhança e densidades a posteriori. As expressões obtidas foram bastante simples, sem grandes dificuldades no trato matemático e de fácil implementação computacional. As técnicas aqui apresentadas podem ser uma ferramenta muito poderosa no desenvolvimento de softs estatísticos.

Como continuidade deste trabalho, pretendemos, ainda, explorar os seguintes tópicos em projetos de pesquisa futuros:

1. Fazer uma análise Bayesiana avaliando o efeito das reparametrizações sobre densidades a posteriori, considerando densidades a priori informativas para a confiabilidade.

2. Pretendemos, também, explorar as reparametrizações apresentadas nas aproximações de Kass, Tierney e Kadane (1989) para funções não-lineares, e a aproximação de Leonard, Hsu e Tsui (1988) para encontrar densidades a posteriori marginais para $R\left(t_{0}\right)$ e $\phi_{G J}(\lambda)$;

3. Considerando a densidade a posteriori conjunta $p(r, \beta /$ dados $)$, obtida a partir de uma densidade a priori não-informativa para $R\left(t_{0}\right)$ e $\beta$, pretendemos aplicar o método de Laplace para aproximação de integrais (ver apêndice $D$ ) para obter a densidade a posteriori marginal de $R\left(t_{0}\right)$ e a esperança a posteriori $E\left[R\left(t_{0}\right) /\right.$ dados $]$, assumindo diversos valores de $t_{0}$. Além disso, ainda usando o método de Laplace, 
pretendemos obter a esperança a posteriori de $R\left(t_{0}\right)$ na parametrização $\left(\phi_{G J} ; \theta\right)$, considerando a densidade a posteriori conjunta $p\left(\phi_{G J}, \theta /\right.$ dados $)$, para avaliar o ganho em precisão na nova parametrização.

4. Pretendemos ilustrar o método proposto para sistemas $k$-out-of- $m$.

5. Poderíamos considerar a função de perda assimétrica Linex, introduzida por Zellner (1986) e estudada por Basu e Ebrahini (1988), como uma alternativa à função de perda quadrática, procurando evitar o problema da superestimação da confiabilidade $R\left(t_{0}\right)$, daí, verificar o efeito das reparametrizações consideradas neste trabalho. 


\section{Referências Bibliográficas.}

ACHCAR, J. A. (1993) - Some Aspects of Reparametrizations for Inferences of kout-of-m System Reliability in the Independente Exponential Case. - Notas do ICMSC, 121 - USP São Carlos.

ACHCAR, J. A. (1993) - Some Aspects of Reparametrization for Statistical Models Notas do ICMSC, Série Estatística, 001 - USP São Carlos.

ACHCAR, J. A. E DIAS, T. C. M., (1990) - Accelereted Tests with an Exponential Distribution: a Beyesian Approach with the Power Rule Model and Type II Censored Data. - Notas do ICMSC, 67 - USP São Carlos.

ACHCAR, J. A. E FOGO, J. C. (1993) - Some Useful Reparametrizations for the Reliability Function Considering the Exponential Distribution and Censored Lifetime Data. - Notas do ICMSC, Série Estatística, 005 - USP São Carlos.

ACHCAR, J. A. E FOGO, J. C. (1994) - An Useful Reparametrization for the Reliability in the Weibull Case. - Notas do ICMSC, Série Estatística, 005 - USP São Carlos.

ACHCAR, J. A. E LOUZADA-NETO, F., (1992) - A Beyesian Approach for Accelerated Life Tests Considering the Weibull Distribution. - Computational Statististics - 7:355-369. 
ACHCAR, J. A. E SMITH, A. F. M. (1990) - Aspects of Reparametrization in Aproximate Bayesian Inference, Essays in Honour of G. A. Barnard. - Ed. J.Hodges, pp. 439-452 - Amsterdam, North Holland.

ANSCOMBE, F. J. (1964) - Normal Likelihood Functions. - Ann. Inst. Stat. Math., 16, 1-19.

ARANDA-ORDAZ, F. J. (1981) - On Two Families of Tranformation to Additivity for Binary Response Data. - Biometrika, 68, 357-363.

ATKINSON, A. C. (1985) - Plots, tranformations and Regression. - Oxford: Claredon Press.

BARLOW, R. E. E PROSCHAN, F. (1979) - Inference for the Exponential Life Distribution. - Research Report ORC 79-16 - Operations Research Center, University of California, Berkeley.

BASU, A. P. (1964) - Estimates of Reliability for Some Distributions Useful in Life Testing. - Technometrics; 6, 215-219.

BASU, A. P. (1975) - Satistical Information and Likelihood - Sankkya, 37, A, 1-71.

BASU, A. P. E EBRAHIMI, N. (1988) - Bayesian Approach to Life Testing and Realiability Estimation Using Asymmetric Loss Function. - Technical Report 144, Department of Statistics - University of Missouri, Columbia. 
BASU, A. P. E EL MAWAZINY, A. H. (1978) - Estimates of Reliability of k-outof-m Structures in the Independent Exponential Case. - JASA, 73, 850-854.

BATES, E. M. L. E WATTS, D. G. (1980) - Relative Curvature Measures of Nonlinearity. - JRSS, B, 42, 1-25.

BICKEL, P. J. E DOKSUM, K. A. (1977) - Mathematical Statistics. Basic Ideas and Selected Topics. - San Francisco, Holden. Day.

BOLFARINE, H. RODRIGUES, J. E ACHCAR, J. A. (1991) - Análise de Sobrevivência - Notas do Minicurso da II Escola de Modelos de Regressão, IMUFRJ - Rio de Janeiro .

BOX, G. E. P. E COX, D. R. (1964) - An Analysis of Transformations (with discussion). - JRSS, B 26, 211-252.

BOX, G. E. P. E TIAO, G. C. (1973) - Bayesian Inference in Statistical Analysis. Reading, MA: Addison-Wesley.

CHAO, A. (1981) - Approximate Mean Squared Errors of Stimators of Reliability for k-out-of-m Systems in the Independent Exponential Case. - JASA, 76, 720-724.

GUERRERO, V. M. E JOHNSON, R. A. (1982) - Use of the Box-Cox Transformation with Binary Response Models. - Biometrika, 69, 309-314.

HILLS, S. E. E SMITH, A. F. M. (1993) - Diagnostic Plots for Improved Parametrization in Bayesian Inference. - Biometrika, 80, 61-74. 
KALbFleisCH, J. D. E PRENTICE, R. L. (1980) - The Statistical Analysis of Failure Time Data. - Wiley, New York.

KASS, R. E. E SLATE, E. H. (1992) - Reparametrization and Diagnostics of Posterior Nonnormality. - In Bayesian Statistics 4, Ed. J.M. Bernardo, J.O. Berger, A.P. Dawid and A.F.M. Smith, pp. 289-306 - Oxford University Press.

KLEIN, J. P. E BASU, A. P. (1981) - Weibull Accelerated Life Tests when there are Competing Causes of Failure. - Communications in Statistics, A10, 20, 2073 - 2100.

LAWLESS, J. F. (1982) - Statistical Model and Methods for Lifetime Data. - New York, John Wiley \& Sons.

LEITE, J. G. E SINGER, J. M. (1990) - Métodos Assintóticos em Estatística. Fundamentos e Aplicações. - Notas do Minicurso do IX SINAPE, IME USP, São Paulo.

LEONARD, T. HSU, J. S. J. E TSUI, K. W. (1989) - Bayesian Marginal Inferences. - JASA 84,1051-1058.

LOUZADA-NETO F. (1991) - Testes de Sobrevivência Acelerados: Uma Análise Bayesiana do Modelo de Eyring. - Dissertação de Mestrado em Ciências de Computação e Matemática Computacional - ICMSC USP- São Carlos.

MARTZ, H. F. E WALLER, R. A. (1982) - Bayesian Reliability Analysis. - New York, John Wiley \& Sons. 
MILLER, R. G. (1981) - Survival Analysis. - New York, John Wiley \& Sons.

MOSTELlER, F. E TUKEY, J. W. (1977) - Data Analysis and Regression. Reading, Mass: Addison-Wesley.

NAYLOR, J. C. E SMITH, A. F. M. (1982) - Applications of a Method for the Efficient Computation of Posterior Distributions. - Applied Statistics, 31, 214-225.

PUGH, E. L. (1963) - The Best Estimate of Reliability in the Exponential Case. Operations Research, 11, 56-62.

RUTEMILLER, H. C. (1966) - Point Estimation of Reliability of a System Comprised of $k$ Elements from de same Exponential Distribution. - JASA, 61, 1029-1032.

SHOOMAN, M. L. (1968) - Probabilistic Reliability. An Engineering Approach. McGraw-Hill, New York.

SPROTT, D. A. (1973) - Normal Likelihoods and Their Relation to Large Sample Theory of Estimation. - Biometrika, 60,3, 457-465.

SPROTT, D. A. (1980) - Maximum Likelihood in Small Samples: Estimation in the Presence of Nuisance Parameters. - Biometrika, 67, 515-523.

TIERNEY, L. E KADANE, J. B. (1986) - Accurate Aproximations for Posterior Moments and Marginal Densities. - JASA, 81, 82-86. 
TIERNEY, L. KASS, R. E. E KADANE, J. B. (1989a) - Approximate Marginal Densities of Nonlinear Functions. - Biometrika, 76, 425-433.

ZACKS, S. E EVEN, M. (1966) - The Efficiences in Small Samples of the Maximun Likelihood and Best Unbiased Estimators of Reliability Functions. - JASA, 61, 1033-1051.

ZELLNER, A. (1986) - Bayesian Estimation and Prediction Using Assymetric Loss Function. - JASA, 81, 446-451. 


\section{Apêndice A}

\section{DERIVADAS DO LOGARITMO DA FUNÇÃO DE VEROSSIMILHANÇA $l\left(\phi_{G J}\right)$ LOCALMENTE NO ESTIMADOR DE MÁXIMA VEROSSIMILHANÇA $\hat{\phi}_{G J}$.}

O logaritmo da função de verossimilhança para $\phi_{G J}=\phi_{G J}(\lambda)$, considerando o modelo exponencial, (ver 3.14) é dado por:

$$
l\left(\phi_{G J}\right)=-d \ln t+d \ln \left[B\left(\phi_{G J}\right)\right]-\frac{T}{t_{0}} B\left(\phi_{G J}\right)
$$

onde $B\left(\phi_{G J}\right)=\ln \left[1+A\left(\phi_{G J}\right)\right]$ e $A\left(\phi_{G J}\right)=\left(1+\phi_{G J}\right)^{-1 / \lambda}$.

As três primeiras derivadas de $l\left(\phi_{G J}\right)$ são dadas por:

$$
\begin{aligned}
& l^{\prime}\left(\phi_{G J}\right)=d \frac{B^{\prime}\left(\phi_{G J}\right)}{B\left(\phi_{G J}\right)}-\frac{T}{t_{0}} B^{\prime}\left(\phi_{G J}\right) \\
& l^{\prime \prime}\left(\phi_{G J}\right)=d \frac{B^{\prime \prime}\left(\phi_{G J}\right)}{B\left(\phi_{G J}\right)}-d\left[\frac{B^{\prime}\left(\phi_{G J}\right)}{B\left(\phi_{G J}\right)}\right]^{2}-\frac{T}{t_{0}} B^{\prime \prime}\left(\phi_{G J}\right) \\
& l^{\prime \prime \prime}\left(\phi_{G J}\right)=d \frac{B^{\prime \prime \prime}\left(\phi_{G J}\right)}{B\left(\phi_{G J}\right)}-3 d \frac{B^{\prime}\left(\phi_{G J}\right) B^{\prime \prime}\left(\phi_{G J}\right)}{\left[B\left(\phi_{G J}\right)\right]^{2}}+2 d\left[\frac{B^{\prime}\left(\phi_{G J}\right)}{B\left(\phi_{G J}\right)}\right]^{3}-\frac{T}{t_{0}} B^{\prime \prime \prime}\left(\phi_{G J}\right)
\end{aligned}
$$


onde: $B^{\prime}\left(\phi_{G J}\right)=\frac{A^{\prime}\left(\phi_{G J}\right)}{\left[1+A\left(\phi_{G J}\right)\right]}$

$$
\begin{aligned}
& B^{\prime \prime}\left(\phi_{G J}\right)=\frac{A^{\prime \prime}\left(\phi_{G J}\right)}{\left[1+A\left(\phi_{G J}\right)\right]}-\left[\frac{A^{\prime}\left(\phi_{G J}\right)}{1+A\left(\phi_{G J}\right)}\right]^{2} \\
& B^{\prime \prime \prime}\left(\phi_{G J}\right)=\frac{A^{\prime \prime \prime}\left(\phi_{G J}\right)}{\left[1+A\left(\phi_{G J}\right)\right]}-3 \frac{A^{\prime}\left(\phi_{G J}\right) A^{\prime \prime}\left(\phi_{G J}\right)}{\left[1+A\left(\phi_{G J}\right)\right]^{2}}+2\left[\frac{A^{\prime}\left(\phi_{G J}\right)}{1+A\left(\phi_{G J}\right)}\right]^{3} \\
& A^{\prime}\left(\phi_{G J}\right)=-\frac{1}{\lambda}\left(1+\phi_{G J}\right)^{-(1 / \lambda+1)} \\
& A^{\prime \prime}\left(\phi_{G J}\right)=\frac{1}{\lambda}\left(\frac{1}{\lambda}+1\right)\left(1+\phi_{G J}\right)^{-(1 / \lambda+2)} \\
& A^{\prime \prime \prime}\left(\phi_{G J}\right)=-\frac{1}{\lambda}\left(\frac{1}{\lambda}+1\right)\left(\frac{1}{\lambda}+2\right)\left(1+\phi_{G J}\right)^{-(1 / \lambda+3)}
\end{aligned}
$$

Portanto, as segunda e terceira derivadas de $l\left(\phi_{G J}\right)$ calculadas no estimador de máxima verossimilhança $\hat{\phi}_{G J}=\left(\mathrm{e}^{d t_{0} / T}-1\right)^{-\lambda}-1$, são dadas por:

$$
\begin{aligned}
& l^{\prime \prime}\left(\hat{\phi}_{G J}\right)=-\frac{T^{2} \mathrm{e}^{-2 d t_{0} / T}}{d \lambda^{2} t_{0}^{2}}\left(\mathrm{e}^{d t_{0} / T}-1\right)^{2(\lambda+1)} \\
& l^{\prime \prime \prime}\left(\hat{\phi}_{G J}\right)=\frac{T^{2}\left(\mathrm{e}^{d t_{0} / T}-1\right)^{3(\lambda+1)}}{d \lambda^{2} t^{2} \mathrm{e}^{2 d t_{0} / T}}\left[\frac{3\left(1+\lambda^{-1} \mathrm{e}^{-d t_{0} / T}\right)}{\left(\mathrm{e}^{d t_{0} / T}-1\right)}-\frac{2 T}{d \lambda t_{0} \mathrm{e}^{d t_{0} / T}}\right]
\end{aligned}
$$




\section{Apêndice B}

\section{DERIVADAS DO LOGARITMO DA FUNÇÃO DE VEROSSIMILHANÇA $l\left(\phi_{A}\right)$ LOCALMENTE NO ESTIMADOR DE MÁXIMA VEROSSIMILHANÇA $\hat{\boldsymbol{\phi}}_{A O}$.}

O logaritmo da função de verossimilhança para $\phi_{A O}=\phi_{A O}(\lambda)$, considerando o modelo exponencial, (ver 3.22) é dado por:

$$
l\left(\phi_{A O}\right)=-d \ln t+d \ln \left[D\left(\phi_{A O}\right)\right]-\frac{T}{t_{0}} D\left(\phi_{A O}\right),
$$

onde $D\left(\phi_{A O}\right)=\ln \left[1+C\left(\phi_{A O}\right)\right]$ e $C\left(\phi_{A O}\right)=\left(\frac{1-\phi_{A O}}{1+\phi_{A O}}\right)^{1 / \lambda}$.

As três primeiras derivadas de $l\left(\phi_{A O}\right)$ são dadas por:

$$
\begin{aligned}
& l^{\prime}\left(\phi_{A O}\right)=d \frac{D^{\prime}\left(\phi_{A O}\right)}{D\left(\phi_{A O}\right)}-\frac{T}{t_{0}} D^{\prime}\left(\phi_{A O}\right), \\
& l^{\prime \prime}\left(\phi_{A O}\right)=d \frac{D^{\prime \prime}\left(\phi_{A O}\right)}{D\left(\phi_{A O}\right)}-d\left[\frac{D^{\prime}\left(\phi_{A O}\right)}{D\left(\phi_{A O}\right)}\right]^{2}-\frac{T}{t_{0}} D^{\prime \prime}\left(\phi_{A O}\right) \\
& l^{\prime \prime \prime}\left(\phi_{A O}\right)=d \frac{D^{\prime \prime \prime}\left(\phi_{A O}\right)}{D\left(\phi_{A O}\right)}-3 d \frac{D^{\prime}\left(\phi_{A O}\right) D^{\prime \prime}\left(\phi_{A O}\right)}{\left[D\left(\phi_{A O}\right)\right]^{2}}+2 d\left[\frac{D^{\prime}\left(\phi_{A O}\right)}{D\left(\phi_{A O}\right)}\right]^{3}-\frac{T}{t_{0}} D^{\prime \prime \prime}\left(\phi_{A O}\right)
\end{aligned}
$$


Apêndices

121

onde: $D^{\prime}\left(\phi_{A O}\right)=\frac{C^{\prime}\left(\phi_{A O}\right)}{\left[1+C\left(\phi_{A O}\right)\right]}$

通

$$
\begin{aligned}
& D^{\prime \prime}\left(\phi_{A O}\right)=\frac{C^{\prime \prime}\left(\phi_{A O}\right)}{\left[1+C\left(\phi_{A O}\right)\right]}-\left[\frac{C^{\prime}\left(\phi_{A O}\right)}{1+C\left(\phi_{A O}\right)}\right]^{2} \\
& D^{\prime \prime \prime}\left(\phi_{A O}\right)=\frac{C^{\prime \prime \prime}\left(\phi_{A O}\right)}{\left[1+C\left(\phi_{A O}\right)\right]}-3 \frac{C^{\prime}\left(\phi_{A O}\right) C^{\prime \prime}\left(\phi_{A O}\right)}{\left[1+C\left(\phi_{A O}\right)\right]^{2}}+2\left[\frac{C^{\prime}\left(\phi_{A O}\right)}{1+C\left(\phi_{A O}\right)}\right]^{3} \\
& C^{\prime}\left(\phi_{A O}\right)=-\frac{2}{\lambda} \frac{C\left(\phi_{A O}\right)}{\left(1-\phi_{A O}^{2}\right)} \\
& C^{\prime \prime}\left(\phi_{A O}\right)=\frac{4}{\lambda} \frac{C\left(\phi_{A O}\right)}{\left(1-\phi_{A O}^{2}\right)^{2}}\left(\frac{1}{\lambda}-\phi_{A O}\right) \\
& C^{\prime \prime \prime}\left(\phi_{A O}\right)=\frac{4}{\lambda} \frac{C\left(\phi_{A O}\right)}{\left(1-\phi_{A O}^{2}\right)^{2}}\left[\frac{2}{\left(1-\phi_{A O}^{2}\right)}\left(2 \phi_{A O}-\frac{1}{\lambda}\right)\left(\frac{1}{\lambda}-\phi_{A O}\right)-1\right]
\end{aligned}
$$

Portanto, as segunda e terceira derivadas de $l\left(\phi_{A O}\right)$ calculadas no estimador de máxima verossimilhança,

$$
\hat{\phi}_{A O}=\frac{1-\left(\mathrm{e}^{d t_{0} / T}-1\right)^{\lambda}}{1+\left(\mathrm{e}^{d t_{0} / T}-1\right)^{\lambda}},
$$


são dadas por:

$$
\begin{aligned}
& l^{\prime \prime}\left(\hat{\phi}_{A O}\right)=-\frac{4 T^{2} \mathrm{e}^{-2 d t_{0} / T}\left(\mathrm{e}^{d t_{0} / T}-1\right)^{2}}{d \lambda^{2} t_{0}^{2}\left(1-\hat{\phi}_{A O}^{2}\right)^{2}} \\
& l^{\prime \prime \prime}\left(\hat{\phi}_{A O}\right)=\frac{8 T^{2} \mathrm{e}^{-2 d t_{0} / T}\left(\mathrm{e}^{d t_{0} / T}-1\right)^{2}}{d \lambda^{2} t_{0}^{2}\left(1-\hat{\phi}_{A O}^{2}\right)^{3}}\left[\frac{3 \mathrm{e}^{-d t_{0} / T}}{\lambda}-3 \hat{\phi}_{A O}-\frac{2 T}{d \lambda t_{0}}+\frac{2 T \mathrm{e}^{-d t / T}}{d \lambda t_{0}}\right] .
\end{aligned}
$$




\section{Apêndice C}

\section{O MÉTODO DELTA.}

Seja $Y$ uma variável aleatória com média $\mu$ e variância $\sigma^{2}$. Supor que estamos interessados em determinar a distribuição de uma função $g(Y)$ continua e derivável num intervalo que contém $\mu$, com $g^{\prime}(Y) \neq 0$. Se $Y^{a} \sim N\left(\mu ; \sigma^{2}\right)$, então:

$$
g(Y) \stackrel{a}{\sim} N\left\{g(\mu) ;\left[g^{\prime}(\mu)\right]^{2} \sigma^{2}\right\}
$$

Em sua versão multivariada, seja $\mathbf{Y}=\left(Y_{1}, Y_{2}, \ldots, Y_{m}\right)$ um vetor de variáveis aleatórias com médias $\mu=\left(\mu_{1}, \mu_{2}, \ldots, \mu_{m}\right)$ e matriz de covariâncias

$$
\Sigma=\left[\begin{array}{cccc}
\sigma_{Y_{1}}^{2} & \sigma_{Y_{1}, Y_{2}} & \cdots & \sigma_{Y_{1}, Y_{m}} \\
& \sigma_{Y_{2}}^{2} & \cdots & \sigma_{Y_{2}, Y_{m}} \\
& & \ddots & \vdots \\
& & & \sigma_{Y_{m}}^{2}
\end{array}\right] .
$$

Se $\mathbf{Y} \stackrel{a}{\sim} N(\boldsymbol{\mu} ; \Sigma)$ então, a distribuição de uma função $g(\mathbf{Y})$ é dada por:

$$
g(\mathbf{Y}) \stackrel{a}{\sim} N\left\{g(\mu) ; \mathbf{H}^{\prime} \Sigma \mathbf{H}\right\}
$$

onde $\mathbf{H}^{\prime}=\left(\frac{\partial g}{\partial Y_{1}}, \frac{\partial g}{\partial Y_{2}}, \ldots, \frac{\partial g}{\partial Y_{m}}\right),($ ver por exemplo, Leite e Singer, 1990). 


\section{Apêndice D}

\section{O MÉTODO DE LAPLACE PARA APROXIMAÇÃO DE INTEGRAIS.}

Uma das limitações dos métodos Bayesianos nas aplicações em geral, relacionase à resolução de integrais que muitas vezes não apresentam soluções analíticas, exigindo o uso de métodos de aproximação. Neste sentido, um dos métodos mais utilizados em inferência Bayesiana é o método de Laplace para aproximação de integrais (ver Tierney e Kadane, 1986).

Suponha que desejamos resolver a integral em $\theta$, dada por:

$$
I=\int_{\Theta} g(\theta) d \theta
$$

Para obter uma aproximação para (D.1) pelo método de Laplace, fazemos

$$
\int_{\Theta} g(\theta) d \theta=\int_{\Theta} f(\theta) \mathrm{e}^{-n h(\theta)} d \theta
$$

onde $h(\theta)$ é uma função unimodal com máximo em $\hat{\theta}$.

No caso em que $\theta$ é uniparamétrico, $\operatorname{com} f(\theta)=1$, a integral (D.1) é aproximada por:

$$
I \approx(2 \pi)^{1 / 2}\left[n h^{\prime \prime}(\hat{\theta})\right]^{-1 / 2} \mathrm{e}^{-n h(\hat{\theta})} .
$$


No caso multiparamétrico, a aproximação para (D.1) é dada por:

$$
I \approx(2 \pi)^{m / 2}\left\{\operatorname{det}\left[n D^{2} h(\hat{\theta})\right]\right\}^{-1 / 2} \mathrm{e}^{-n h(\hat{\theta})} .
$$

onde $m=$ número de parâmetros e $D^{2} h(\hat{\theta})$ é a matriz Hessiana de $h$, calculada em $\hat{\theta}$, definida por:

$$
D^{2} h(\theta)=\left[\begin{array}{cccc}
\frac{\partial^{2} h}{\partial \theta_{1}^{2}} & \frac{\partial^{2} h}{\partial \theta_{1} \partial \theta_{2}} & \cdots & \frac{\partial^{2} h}{\partial \theta_{1} \partial \theta_{m}} \\
& \frac{\partial^{2} h}{\partial \theta_{2}^{2}} & \cdots & \frac{\partial^{2} h}{\partial \theta_{2} \partial \theta_{m}} \\
& & \ddots & \vdots \\
& & & \frac{\partial^{2} h}{\partial \theta_{m}^{2}}
\end{array}\right]
$$

Em aplicações Bayesianas, com $\theta$ uniparamétrico, supor que desejamos obter a esperança a posteriori de uma função $g(\theta)$, monótona crescente, dada por:

$$
E_{\theta}[g(\theta) / \text { dados }]=\frac{\int_{\Theta} g(\theta) L(\theta) p_{0}(\theta) d \theta}{\int_{\Theta} L(\theta) p_{0}(\theta) d \theta},
$$

onde $L(\theta)$ e $p_{0}(\theta)$ são, repectivamente, a função de verosssimilhança e a densidade a priori de $\theta$. Então, de (D.2), com $f(\theta)=1$, podemos escrever (D.6) por:

$$
E_{\theta}[g(\theta) / \text { dados }]=\frac{\int_{\Theta} \mathrm{e}^{-n h_{*}(\theta)} d \theta}{\int_{\Theta} \mathrm{e}^{-n h(\theta)} d \theta},
$$




$$
\text { onde }-n h_{*}(\theta)=\ln g(\theta)+\ln p_{0}(\theta)+l(\theta) \text { e }-n h(\theta)=\ln g(\theta)+\ln p_{0}(\theta) \text {. }
$$

Portanto, pelo método de Laplace aplicado ao numerador e denominador de (D.7), uma aproximação para a esperança a posteriori (D.6) é dada por:

$$
E_{\theta}[g(\theta) / \text { dados }] \approx\left[\frac{n h_{*}^{\prime \prime}\left(\hat{\theta}_{*}\right)}{n h^{\prime \prime}(\hat{\theta})}\right]^{-1 / 2} \exp \left\{-n h_{*}\left(\theta_{*}\right)+n h(\hat{\theta})\right\}
$$

onde $\hat{\theta}_{*}$ e $\hat{\theta}$ maximizam $-n h_{*}(\theta)$ e $-n h(\theta)$, respectivamente.

Mais detalhes e estudos sobre a precisão da metodologia descrita podem ser encontrados em Tierney e Kadane (1986). 


\section{Apêndice E}

\section{PROGRAMAS DESENVOLVIDOS.}

A seguir apresentaremos alguns programas desenvolvidos na linguagem Turbo Pascal utilizados para os cálculos em nossas análises.

1. Programa para obtenção do valor de $\lambda_{p}$, minimizando a medida de não normalidade de Kass e Slate (2.13) em testes acelerados, considerando o modelo de lei de potência inversa:

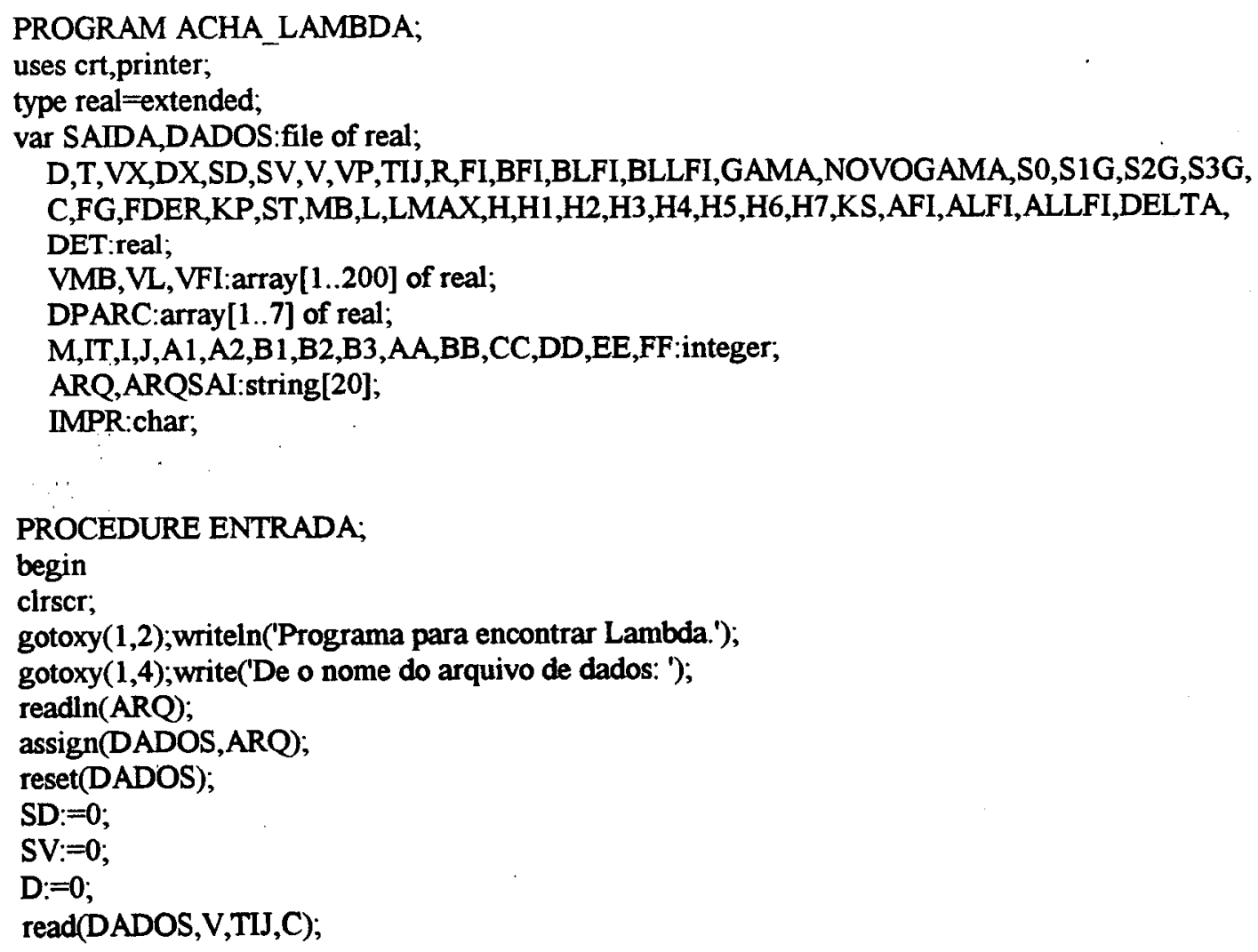




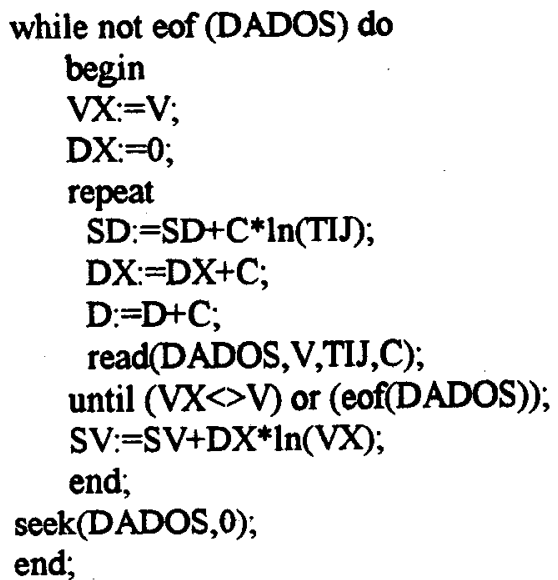

\section{PROCEDURE IMPRIME;}

begin

gotoxy $(1,24)$; write('Prepare a Impressora e pressione < Enter >.');

readln;

writeln(lst,'Programa para encontrar Lambda em Testes ');

writeln(Ist,'Acelerados: Modelo Exponencial.');

writeln(lst);

writeln(lst,' Nivel de Stress em estudo: ',VP:7:2);

writeln(lst);

writeln(lst,'Instante para a Confiabilidade: ',T:7:2);

writeln(lst); writeln(lst);

writeln(lst,' $\quad$ E.M.V. de Gama: ',GAMA:16:8);

writeln(lst);

writeln(Ist,'E.M.V. de R(',T:7:2,'): ',R:16:8);

writeln(lst); writeln(lst);

writeln(lst,' Lambda $\quad F i(G J) \quad m B 2 ')$;

for $J:=1$ to I do

writeln(lst,'\#',J:2,' ',VL[J:12:8,' ',VFI[J]:12:8,' ',VMB[J]:18:12);

writeln(lst); writeln(lst);

writeln(lst,'

Valor de Lambda encontrado = ',VL[M]:12:8);

writeln(lst);

writeln(lst,'

Valor de Fi(GJ) correspondente = ', VFI[M]:12:8);

writeln(lst);

writeln(lst,'

Medida de nao-normalidade: $\mathrm{mB2}(\mathrm{GJ})=$ ',VMB[M]:16:12);

writeln(lst);

writeln(lst,'Medida de nao-normalidade (ORIGINAL): mB2(R) = ',MB:16:12);

writeln(lst,chr(12));

end;

PROCEDURE NEWTON;

begin

IT:=1;

writeln;writeln;

writeln('Iter. Gama $\quad \mathbf{F}$ (Gama)');

repeat

S0: $=0$;

S1G: $=0$;

S2G: $=0$;

S3G: $=0$; 
while not eof (DADOS) do

begin

read(DADOS, V,TIJ,C);

S0:=SO+exp(GAMA*In(V)*TIJ;

S1G: $=S 1 G+\exp (G A M A * \ln (V))^{*} T 1 J * \ln (V / V P)$;

S2G:=S2G+exp(GAMA*ln(V) ${ }^{*}$ TIJ*sqr(ln(V/VP));

S3G:=S3G+exp(GAMA* $\ln (V))^{*} T I J * \ln (V / V P) * \operatorname{sqr}(\ln (V / V P))$;

end;

FG:=SV/D-ln(VP)-S1G/S0;

FDER: $=-\mathrm{S} 2 \mathrm{G} / \mathrm{SO}+\mathrm{sqr}(\mathrm{S} 1 \mathrm{G} / \mathrm{S} 0)$;

NOVOGAMA:=GAMA-FG/FDER;

writeln(IT:2,GAMA:19:8,FG:21:8);

delay (200);

GAMA:=NOVOGAMA;

$\mathrm{IT}:=\mathrm{IT}+1$

seek(DADOS,0);

until $(\mathrm{abs}(\mathrm{FG})<0.0000001)$ or $(\mathrm{IT}>100)$;

$\mathrm{V}:=\exp (\mathrm{GAMA} * \ln (\mathrm{VP}))$;

$\mathrm{R}:=\exp \left(-\mathrm{D}^{*} \mathrm{~T}^{*} \mathrm{~V} / \mathrm{S} 0\right)$;

end;

PROCEDURE MB2;

begin

DPARC $[1]:=\mathrm{H} 4$;

DPARC $[2]:=\mathrm{H} 5$;

DPARC[3]:=H6;

DPARC [4]:=H7;

DPARC [5]:=H1;

DPARC [6]: $=\mathrm{H} 2$;

DPARC[7]:=H3;

$\mathrm{MB}:=0$;

for $\mathrm{AA}:=0$ to 1 do

for $B B:=0$ to 1 do

for $\mathrm{CC}:=0$ to 1 do

for $\mathrm{DD}:=0$ to $1 \mathrm{do}$

for $E E:=0$ to 1 do

for $F F:=0$ to 1 do

begin

$\mathrm{Al}:=\mathrm{AA}+\mathrm{BB}+\mathrm{CC}$;

$\mathrm{A} 2:=\mathrm{DD}+\mathrm{EE}+\mathrm{FF}$;

$\mathrm{B1}:=\mathrm{AA}+\mathrm{BB}$;

$\mathrm{B} 2:=\mathrm{DD}+\mathrm{EE}$;

$\mathrm{B} 3:=\mathrm{CC}+\mathrm{FF}$;

$\mathrm{KS}:=\mathrm{DPARC}[\mathrm{A} 1+1]^{* \mathrm{DPARC}}[\mathrm{A} 2+1]^{* \mathrm{DPARC}}[\mathrm{B} 1+5] * \mathrm{DPARC}[\mathrm{B} 2+5] * \mathrm{DPARC}[\mathrm{B} 3+5]$;

$\mathrm{MB}:=\mathrm{MB}+\mathrm{KS}$;

end;

end;

PROCEDURE MBORIGINAL;

begin

$\mathrm{H} 1:=\mathrm{D} / \mathrm{sqr}(\ln (\mathrm{R}) * \mathrm{R})$;

$\mathrm{H} 2:=\mathrm{KP} /(\ln (\mathrm{R}) * \mathrm{R})$;

$\mathrm{H} 3:=\mathrm{D} * \mathrm{~S} 2 \mathrm{G} / \mathrm{SO}$;

DET:=H1*H3-sqr(H2); 
$\mathrm{C}:=\mathrm{H} 1$;

$\mathrm{H} 1:=\mathrm{H} 3 / \mathrm{DET}$;

$\mathrm{H} 2:=\mathrm{H} 2 / \mathrm{DET}$;

$\mathrm{H} 3:=\mathrm{C} / \mathrm{DET}$;

$H 4:=2 * D /(\operatorname{sqr}(\ln (\mathrm{R}) * \mathrm{R}) * \ln (\mathrm{R}) * \mathrm{R})+3 * \mathrm{D} /(\operatorname{sqr}(\ln (\mathrm{R}) * \mathrm{R}) * \mathrm{R})$;

$\mathrm{H} 5:=-\mathrm{KP} /(\ln (\mathrm{R}) * \operatorname{sqr}(\mathrm{R}))$;

$\mathrm{H} 6:=\mathrm{S} 2 \mathrm{G} /(\mathrm{R} * \mathrm{~T} * \mathrm{~V})$;

$\mathrm{H} 7:=\mathrm{D} * \mathrm{~S} 3 \mathrm{G} / \mathrm{SO}$;

MB2;

end;

PROCEDURE DERIVADAS;

begin

$F I:=\exp (-L * \ln (1 / R-1))-1$;

$\mathrm{AFI}:=\exp (-\ln (1+\mathrm{FI}) / \mathrm{L})$;

ALFI: $=\exp (-(1+1 / \mathrm{L}) * \ln (1+\mathrm{FI})) / \mathrm{L}$;

ALLFI: $=(1+1 / L) * 1 / L * \exp (-(2+1 / L) * \ln (1+F I))$;

$\mathrm{BFI}:=\ln (1+\mathrm{AFI})$;

BLFI:=ALFI $(1+$ AFI);

BLLFI:=ALLFI/(1+AFI)-sqr(ALFI/(1+AFI));

$\mathrm{H} 1:=\mathrm{D} * \mathrm{sqr}(\mathrm{BLFI} / \mathrm{BFI})$;

$\mathrm{H} 2:=\mathrm{BLFI} * \mathrm{~S} 1 \mathrm{G} /(\mathrm{T} * \mathrm{~V})$;

$\mathrm{H} 3:=\mathrm{BFI} * \mathrm{~S} 2 \mathrm{G} /(\mathrm{T} * \mathrm{~V})$;

DET: $=\mathrm{H} 1 * \mathrm{H} 3-\mathrm{sqr}(\mathrm{H} 2)$;

$\mathrm{C}:=\mathrm{H} 1$;

$\mathrm{H1}:=\mathrm{H} 3 / \mathrm{DET}$;

$\mathrm{H} 2:=\mathrm{H} 2 / \mathrm{DET}$;

$\mathrm{H} 3:=\mathrm{C} / \mathrm{DET}$;

$\mathrm{H} 4:=2 * \mathrm{D} * \mathrm{sqr}(\mathrm{BLFI} / \mathrm{BFI}) *(\mathrm{BLFI} / \mathrm{BFI})-3 * \mathrm{D} * \mathrm{BLLFI}{ }^{* B L F I} / \mathrm{sqr}(\mathrm{BFI})$;

$\mathrm{H} 5:=\mathrm{BLLFI}^{*} \mathrm{~S} 1 \mathrm{G} /\left(\mathrm{T}^{*} \mathrm{~V}\right)$;

$\mathrm{H} 6:=-\mathrm{BLFI}^{*} \mathrm{~S} 2 \mathrm{G} /\left(\mathrm{T}^{*} \mathrm{~V}\right)$;

$\mathrm{H} 7:=\mathrm{BFI}{ }^{*} \mathrm{~S} 3 \mathrm{G} /\left(\mathrm{T}^{*} \mathrm{~V}\right)$;

end;

PROCEDURE ACHALAMBDA;

begin

clrscr;

$\mathrm{L}:=1$;

LMAX:=1;

repeat

$\mathrm{H}:=(\mathrm{LMAX}-\mathrm{L}) / \mathbf{1 0}$;

$\mathrm{I}:=0$;

repeat

DERIVADAS;

MB2;

$\mathrm{I}:=\mathrm{I}+1$;

VL[I]: $=\mathrm{L}$;

VFI[I]: $=$ FI;

$\mathrm{VMB}[\mathrm{I}]:=\mathrm{MB}$;

$\mathrm{L}:=\mathrm{L}+\mathrm{H}$;

until L > LMAX;

clrscr;

$\mathrm{M}:=1$ 

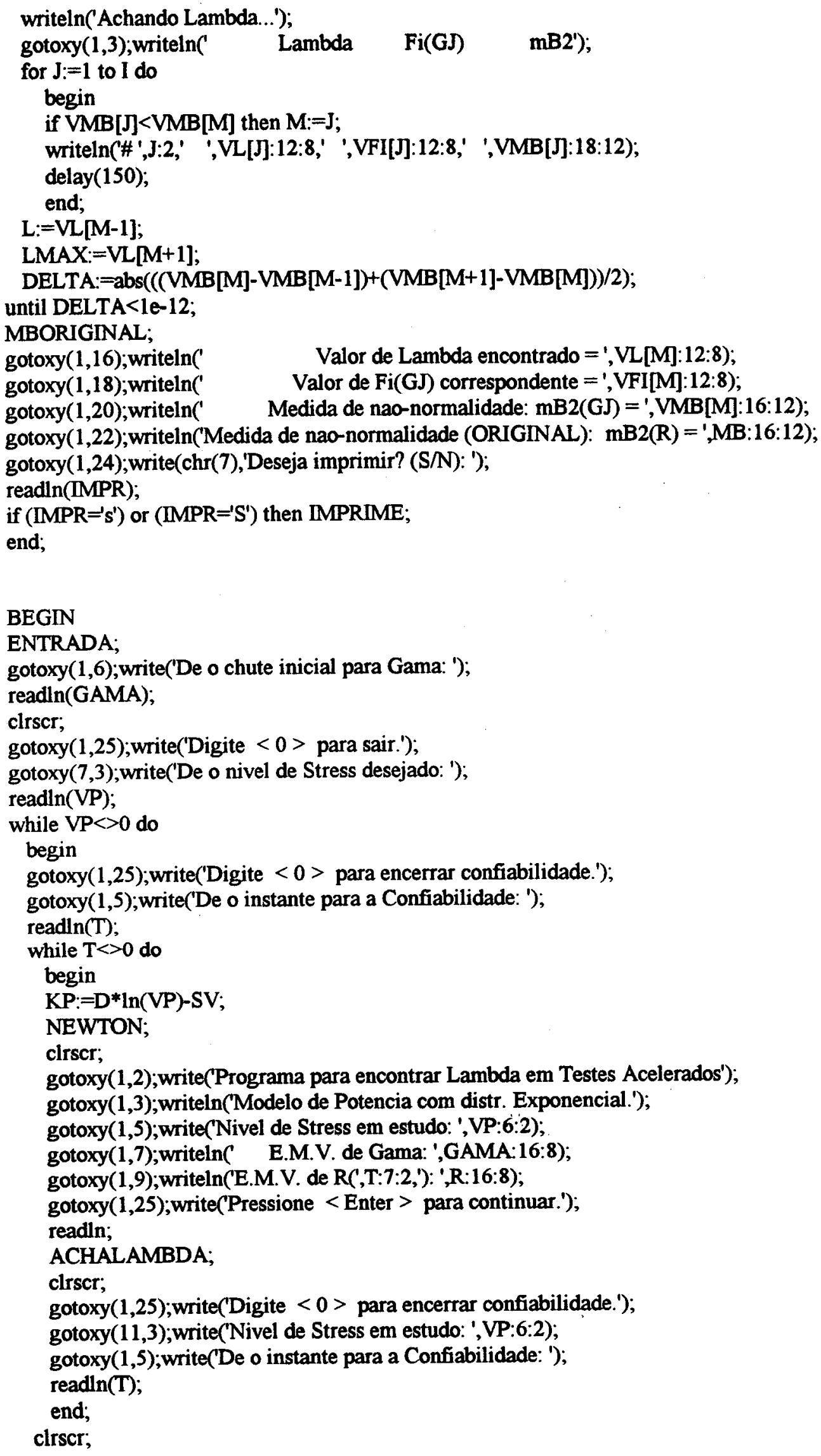
gotoxy(1,25);write('Digite <0 > para sair.');

gotoxy(7,3); write('De o nivel de Stress desejado: ');

readln(VP);

end;

close(DADOS);

end.

Saida do programa:

Programa para encontrar Lambda em Testes Acelerados. Modelo Potencia com Tempos de vida Exponencial.

Nivel de Stress em estudo: 10.00

Instante para a Confiabilidade: 200.00

E.M.V. de Gama: $\quad 0.79945160$

E.M.V. de R( 200.00): $\quad 0.08063303$

\begin{tabular}{|c|c|c|c|}
\hline & Lambda & Fi(GJ) & $\mathrm{mB2}$ \\
\hline & 0.26387712 & -0.47387585 & 0.001346624263 \\
\hline 2 & 0.26387763 & -0.47387650 & 0.001346624213 \\
\hline \# 3 & 0.26387814 & -0.47387716 & 0.001346624172 \\
\hline$\$ 4$ & 0.26387866 & -0.47387781 & 0.001346624148 \\
\hline$\$ 5$ & 0.26387917 & -0.47387847 & 0.001346624118 \\
\hline$\# 6$ & 0.26387968 & -0.47387913 & 0.001346624104 \\
\hline & 0.26388019 & -0.47387978 & 0.001346624100 \\
\hline & 0.26388070 & -0.47388044 & 0.001346624105 \\
\hline & 0.26388122 & -0.47388109 & 0.001346624119 \\
\hline & 0.26388173 & -0.47388175 & 0.001346624142 \\
\hline
\end{tabular}

Valor de Lambda encontrado $=0.26388019$

Valor de $\mathrm{Fi}(\mathrm{GJ})$ correspondente $=\mathbf{- 0 . 4 7 3 8 7 9 7 8}$

Medida de nao-normalidade: $\mathrm{mB2}(\mathrm{GJ})=0.00134662$

Medida de nao-normalidade (ORIGINAL): $\mathrm{mB2}(\mathrm{R})=5.90221117$ 
2. Programa para cálculo da esperança a posteriori pelo método de Laplace, na parametrização de Guerrero e Johnson, considerando o modelo exponencial e uma priori não informativa de Jeffreys para a confiabilidade:

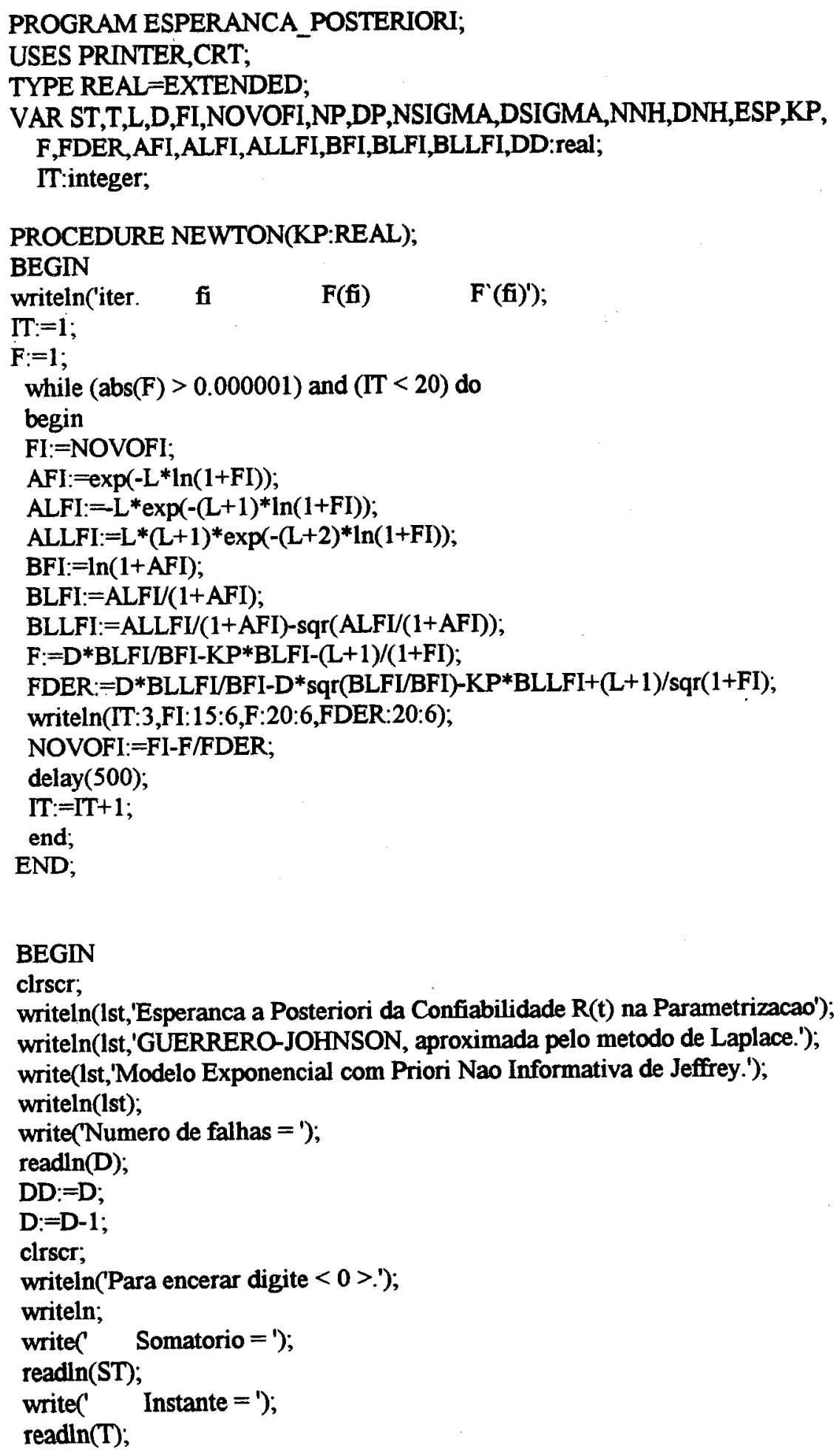




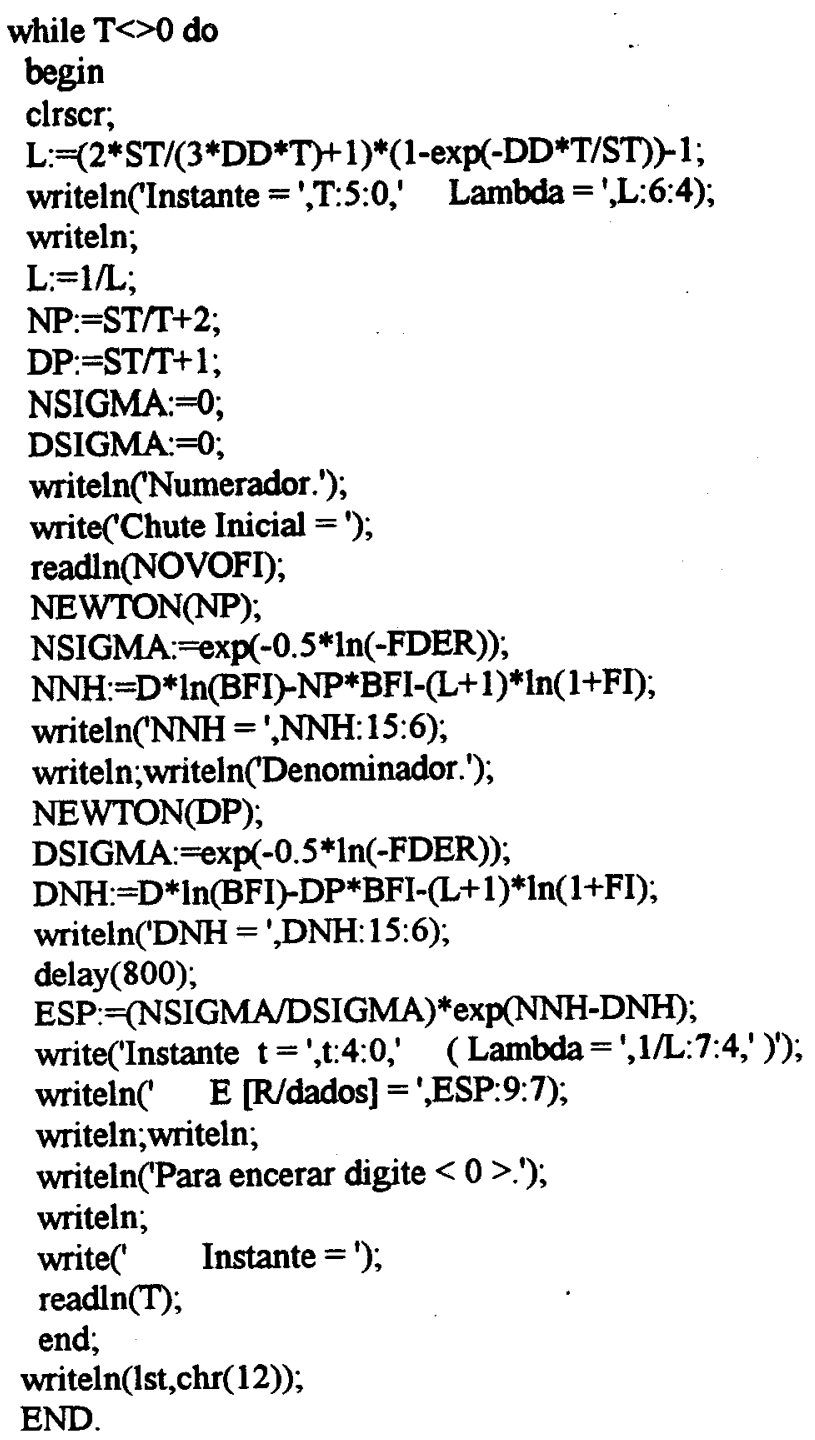

Saida do programa:

Esperanca a Posteriori da Confiabilidade $\mathbf{R}(\mathrm{t})$ na Parametrizacao GUERRERO-JOHNSON, aproximada pelo metodo de Laplace. Modelo Exponencial com Priori Nao Informativa de Jeffrey.

$\begin{array}{llll}\text { Instante } t=5 & (\text { Lambda }=-0.3281) & E[R / \text { dados }]=0.9921348 \\ \text { Instante } t=30 & (\text { Lambda }=-0.3026) & E[R / \text { dados }]=0.9538437 \\ \text { Instante } t=500 & (\text { Lambda }=0.0071) & E[R / \text { dados }]=0.4709958 \\ \text { Instante } t=2000 & (\text { Lambda }=0.1596) & E[R / \text { dados }]=0.0687302\end{array}$


3. Programa para gerar dados para a função de verossimilhança profile em testes acelerados, na parametrização original, $r_{p}$ e $\gamma$, considerando o modelo de lei de potência inversa com tempos de vida exponenciais:

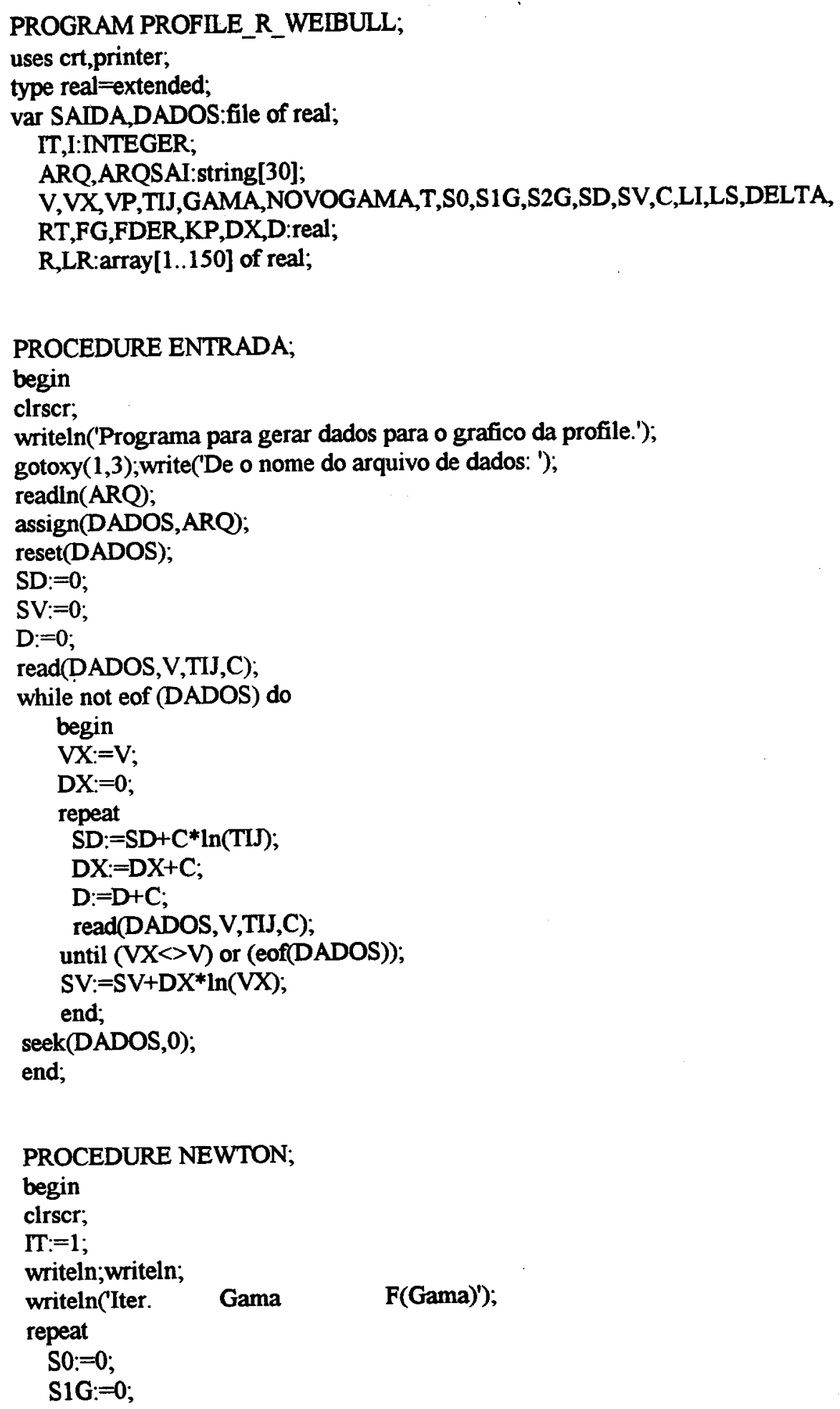




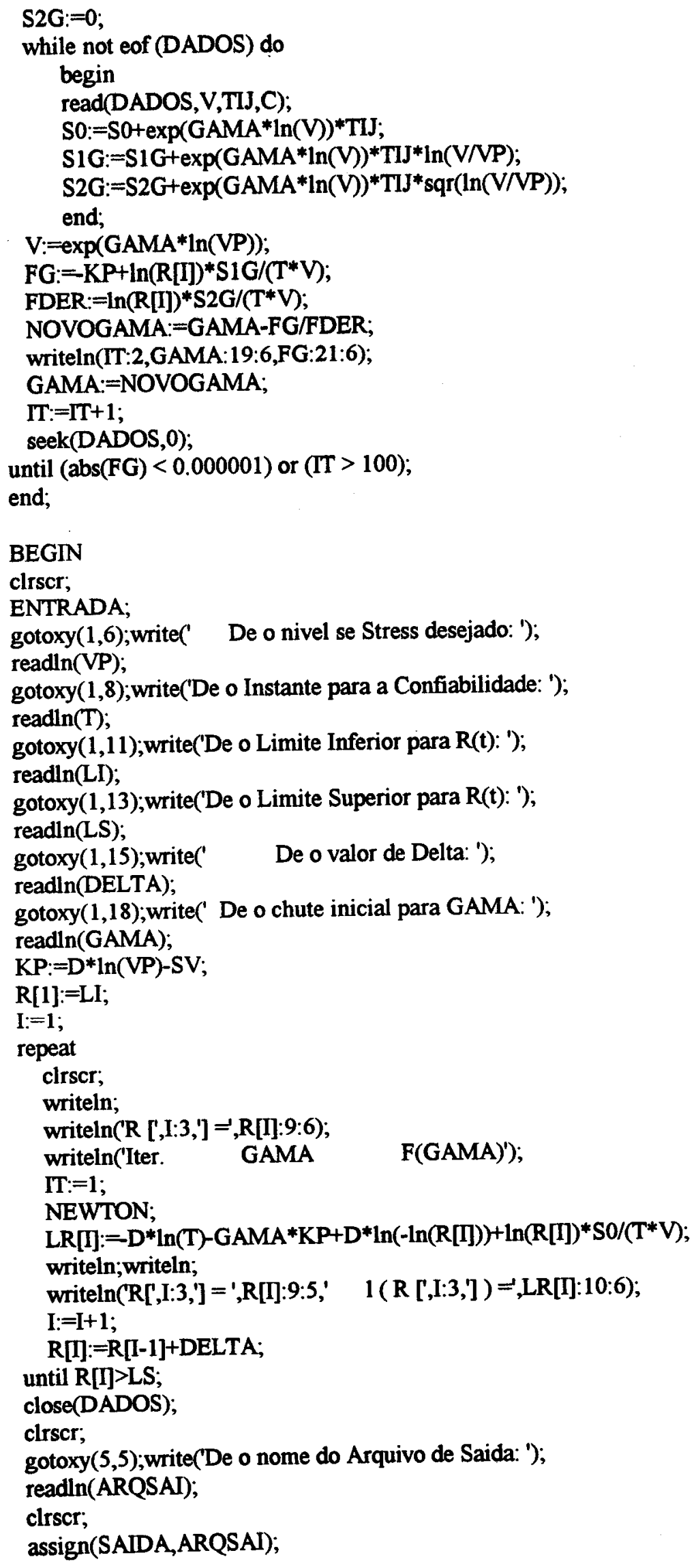




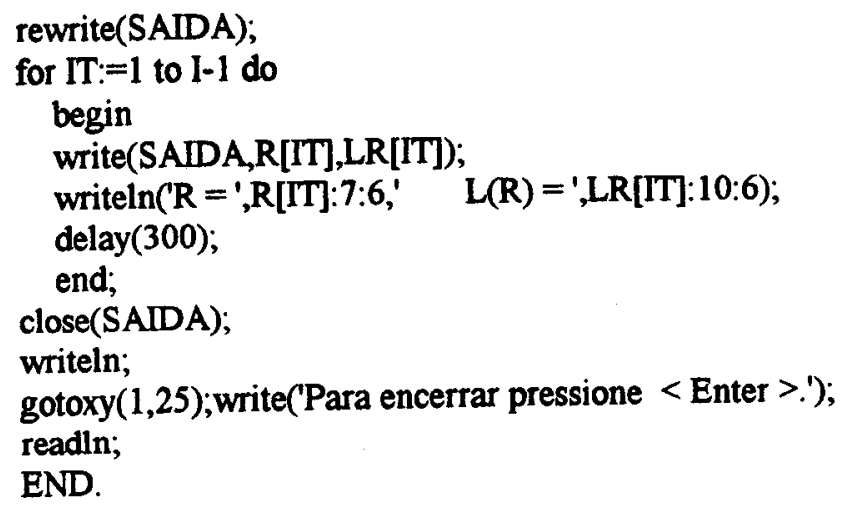


4. Programa para gerar dados para os contornos da função de verossimilhança conjunta, na parametrização $\phi_{G J}(\lambda)$ e $\theta$, considerando o modelo de Weibull para a confiabilidade:

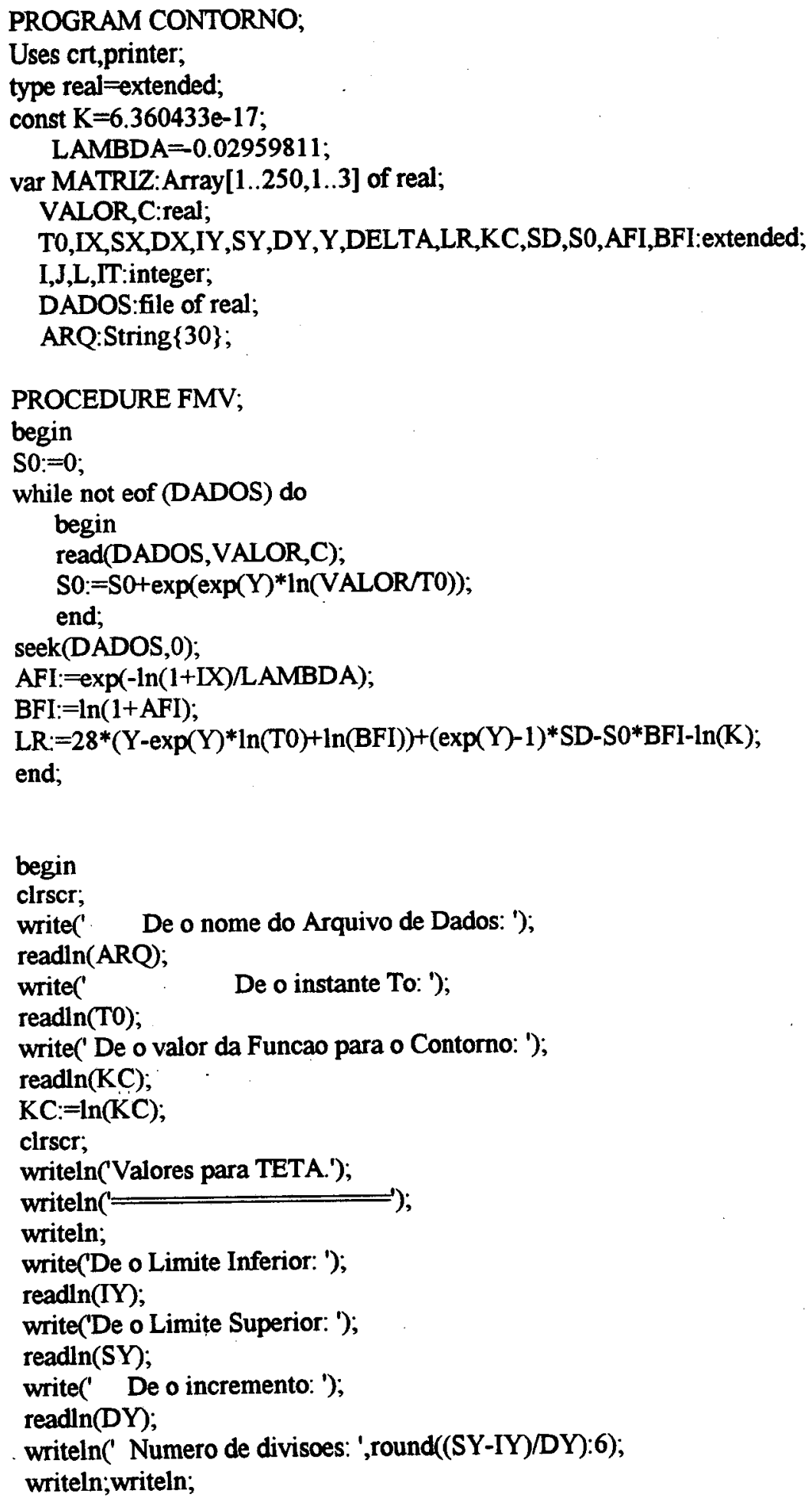




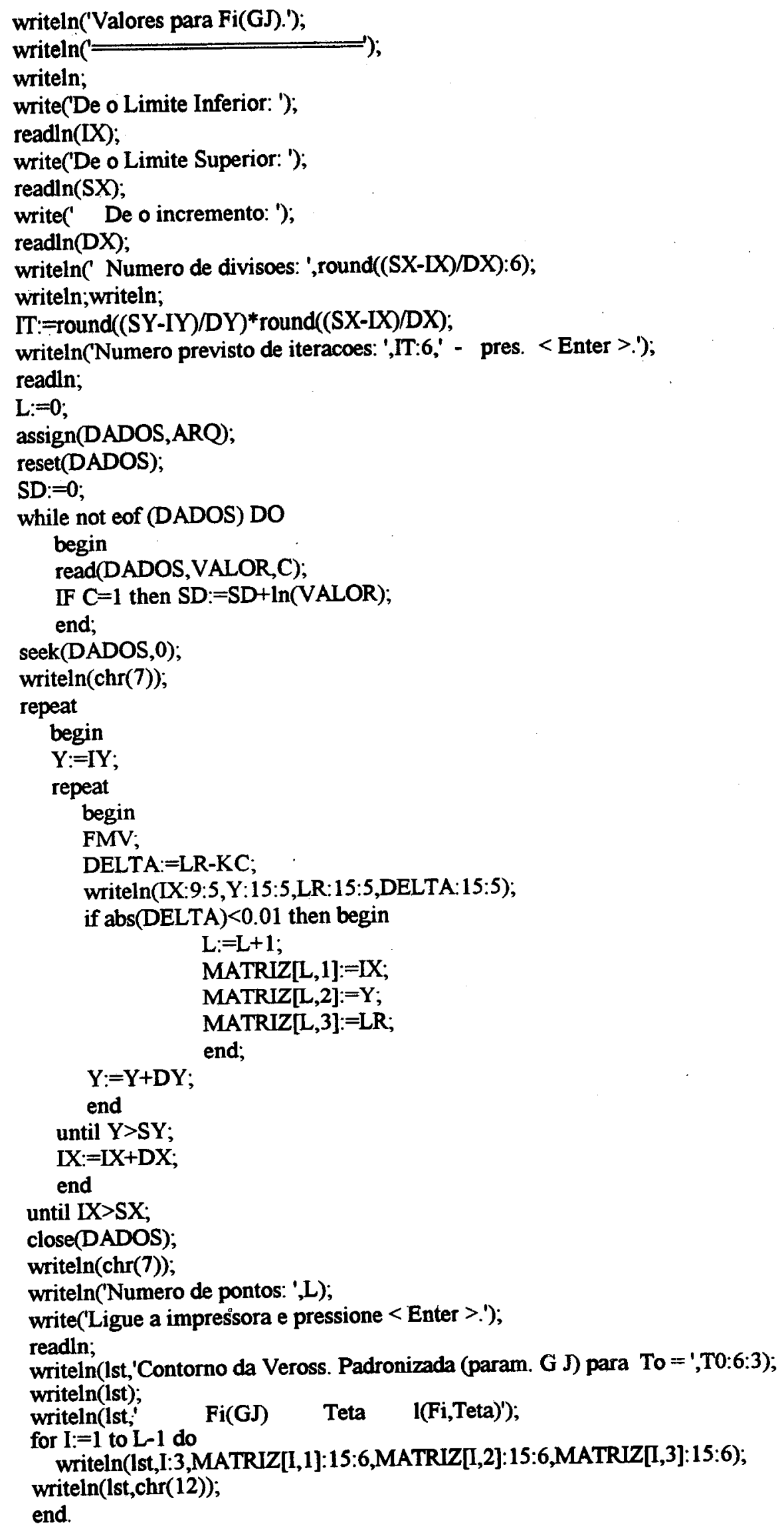

